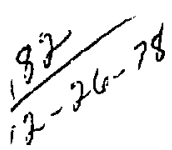

\title{
MISTER
}

\section{DYNSYL: A GENERAL-PURPOSE DYNAMIC SIMULATOR FOR CHEMICAL PROCESSES}

G. K. Patterson

R. B. Rozsia

September 5, 1978

Work performed under the auspices of the US Depariment of

Energy by the UCLLL under contract number $W$-7405-ENG-48

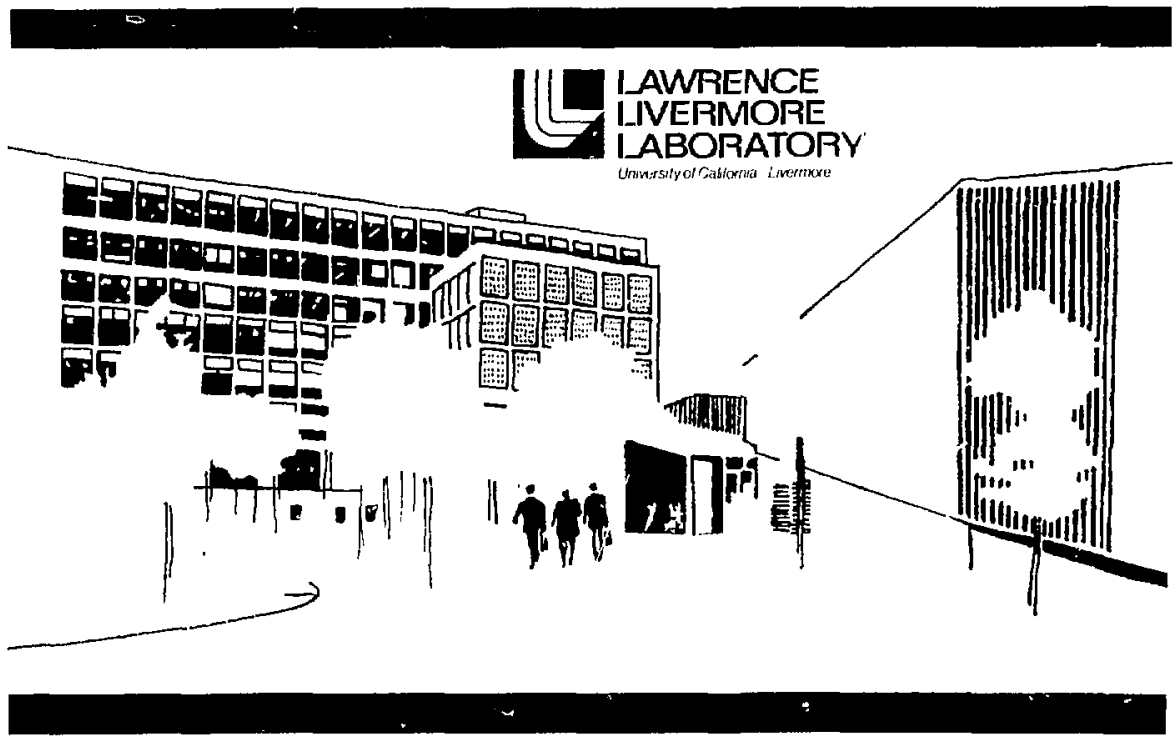




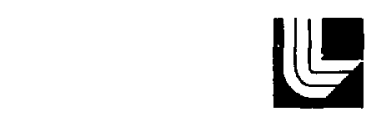

\title{
I_AWRENCE LIVERMORE I_ABORATORY
}

University of Calfornia Livermore.Californa 94550

\section{บCAL. 52561 \\ DYNSYL: A GENERAL-PURPOSE DYNAMIC SIMULATOR FOR CHEMICAL PROCESSES}

\author{
G. K. Patterson* \\ R. B. Rozsa
}

MS. dale: September 5, 1978

"Now with the Department of Chemical Engineering. University of Missouri-Ro!: : Rolla. Missuuri. 


\section{CONTENTS}

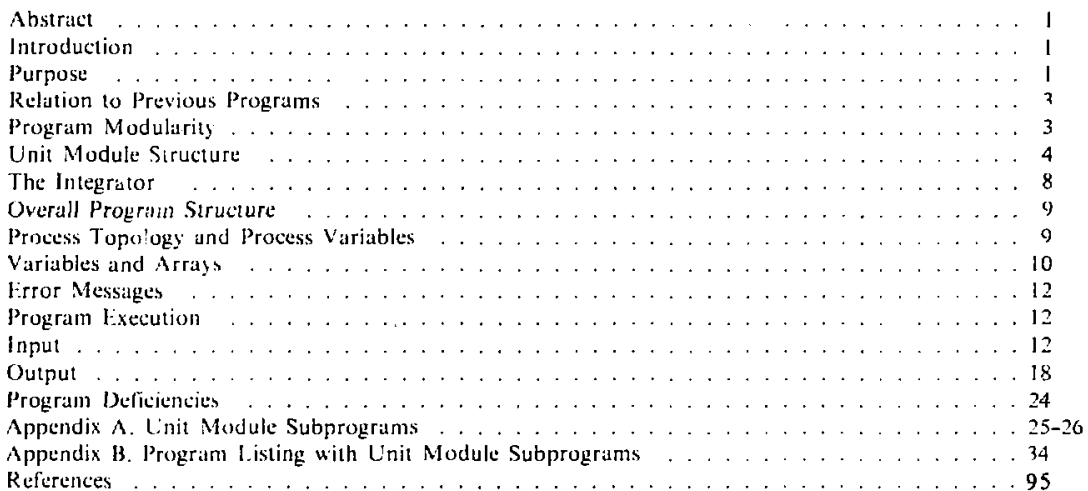




\title{
DYNSYL: A GENERAL-PURPOSE DYNAMIC SIMULATOR FOR CHEMICAL PROCESSES
}

\begin{abstract}
Lawrence Livermore Laboratory is conducting a safeguards program for the Nuclear Regulatory Commission. The goal of the Material Control Project of this program is to evaluate material control and accounting (MCAl methods in plants that handle special nuclear material (SNM). To this end we designed and implemented the dynamic chemical plant simulation program DYNSYL. This program can be used to generate process data or to provide estimates of process performance; it simulates both steady-state and dynamic behavior. The MCA methods that may have to be evaluated range from sophisticated online material trackers such as Kalman filter estimators, to relatively simple naaterial balance procedures. This report describes the overall structure of DYNSYl and includes some exampie problems. The code is still in the experimental stage and revision is continuing.
\end{abstract}

\section{INTRODUCTION}

DYNSYI , a moditication of DYNSYS, ' uses modular program logic to simulate chemical plant dyanmic hehavior. The differential equations gencrated by etach process unil module are timewise-integrated by a stiff equation system integrattor. All equations can be incegraled in al completely coupled mode, or the various units can be simulated in an uncoupled mode. Input data required include in and out process stream numbers. operating parameters (size, rate constants, operation mode. ele.), and stream parameters (flow rate. temperature. pressure. concentrations) for each unit, ats vell as graphical and printed output specilications, and simulation time specifications. Operatur-initiated process changes maly be input by terminat

Oupul results include an input dalta who, all stream parameter and unit parameter values at the end of each time nintertal, and printplot and plotter results for selected stream parameters as a funclion of time.

The program "ils developed to simulate chemical processes in the nuctear linel cycte. The unit subroutines imodulest as aslable are a general. purpose transport unt for equilibrium stage computations with heat Iranefier rliquid-liquid or liquad. vapor). or for stirred-tanh misung and reactoon: at controller with various modes: a pipe: a pump: at highly accuralle saraclur for uranium and platomium coextration or separation in Purex plants: a plutonium precipitator: and a plutomum concentrator. Such simulations proside datal far matcrial aceounting studics. particularly for on-line schemes. and allow studies of dy namic plant operalion either lor assessment or dengn.

\section{PURPOSE}

DYNSYI. hals been designed and implemenled to help evaluale MCA methods in plants that handle SNM. To defect diversion of SNM from chemical processes using MCA methods. the process units and subsections of the plant must first be simulated in detail. Both the steady-state and dynamic belaviors of the chemical process are needed to aceurately evaluate MCA nethods.
The process simulation provides "measured data" for MCA methods evaluations. The MCA method is ollen simulited dynamically in much the same way that the process is simulated. with measured data as the input and probability of detestion of a diversion as the output.

Figure I shows the steps of a MCA nethod evaluation. The process is first dynanically 
NRC/LLL MCA

evaluation data base

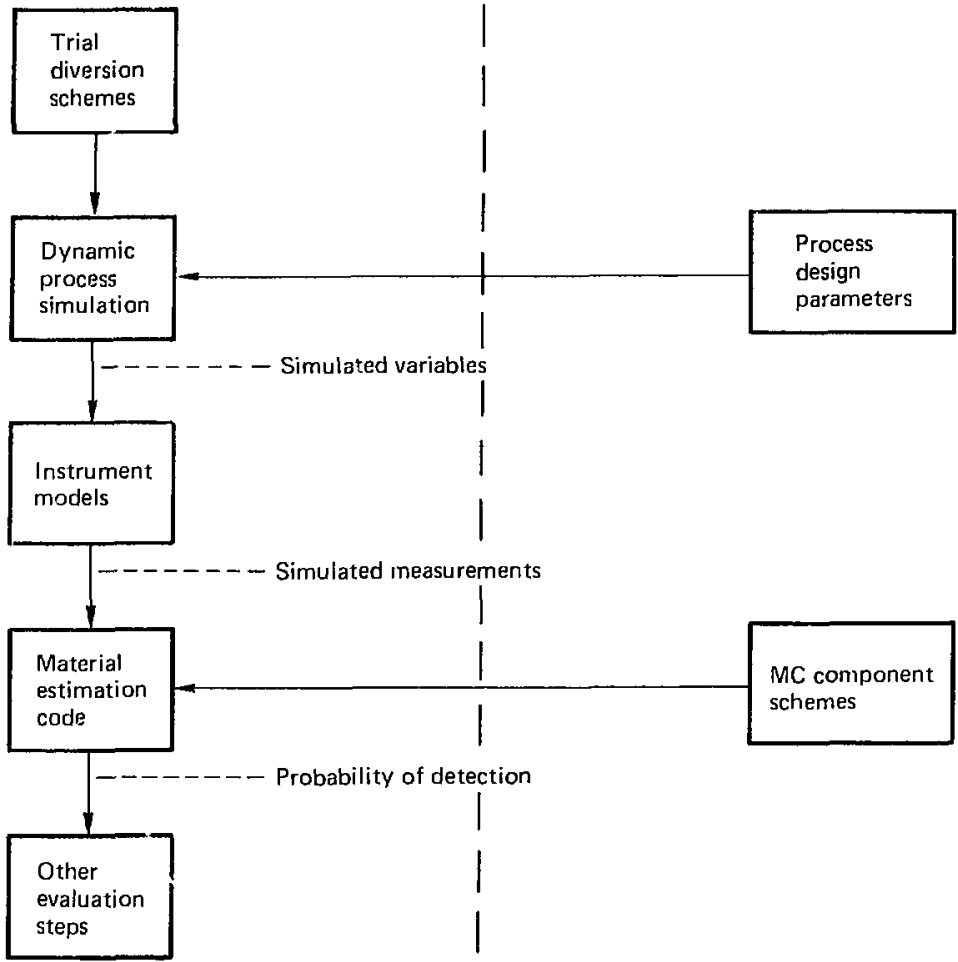

lïg. I. Siteps for evaluating all vic t method.

simulated using validated models of the process units and instruments that provide simulated measurements. These meusured data are then input into the MCA method, and the probability of detection is derived for given levels of material diversion. The material diversions are incorporated into the process simulation as shown in Fig. 1.

As well as providing the basis for MCA evaluations, the dynanic and steady-state process simulations may also be used to chreck design validity and to obtain nominal operating levels for diversion tests. 


\section{RELATION TO PREVIOUS PROGRAMS}

The dynamic chemical plant simulation we have described is a continuous timewise simulation. as opposed to discrete time interval types that simulate inventory and material flows in a plant (i.e., GPSS, 2 GASP, 3 Simscript ${ }^{4}$ ). Continuous dynamic simulations use differential and algebraic models of each of the units in a plant. These models are linked together to represent a numerical simulation of the analog behavior of the overall system.

Previous versions of continuous dynamic plant simulators include PRODYC, 5 DYSCO, 6 REMUS. ${ }^{7}$ DYFLOW ${ }^{8}$ and DYNSYS, the model for DYNSYL. All of these simulation methods use generic models of process units that can be combined to simulate a larger seetion of a plant.

PRODYC interfaces the process unit simulations to the IBM Continuous System Modeling Program (CSMP 9) 10 solve the system of differentiul equations generited. PRODYC, therefore. makes it easier to use CSMP dynamic process simulation. DYSCO is more modular in concept than PRODYC, allowing process flowsheet simulation entirely through input data specifications that consist primarily of a process topology matrix and unit and stream specifications. The code is formulated with process design as the major objective; graphical interaction and ease of process alteration are built-in.

DYNSYS was designed primartly as a batch simulation program with no interactive features included. Lt is otherwise basically the same as DYSCO with a topology matrix, specifications for each unit, and stream specifications that are also the main dynamic (state) variables in the simulation. DYNSYS does, however, account better for coupling effects between units as discussed in the next section.

\section{PROGRAM MODULARITY}

Two basic coneepts are used to design dynamic chemical plant simulation codes: the equationoriented and the modular methods. The equationoriented method (bometimes called continuous system simulation) derives from analog simulation methods (CSMP, ? MIMIC, ${ }^{10}$ LEANS ") that provide solutions to large numbers of simultaneous inturdependen! differentjal equations. The method requires only that all the relevant equations be included. Generally, coupling between equations is included in each solution step.

The modular method uses computer code subroutines (mocules) that compute the differentiais of the state variables associated with the process unit simulated by the module. In most cases, equation solutions for each module are determined independently of the other modules during each time period. Thus, coupling between modules occurs only at the end of the time intervals and not at each step in the integration process as in most equationoriented methods. As noted by Franks, ${ }^{8}$ equationoriented codes may be considered to represent a programming level intermediate between FORTRAN (or other scientific programming language) and modular codes that do not require mathematical formulations.

Both inethods have advantages. Equationoriented codes with complete equation coupling ensure the greatest dynamic sinulation accuracy, particularly for large time steps, but require common storage for all derivative and/or variable values for all integration sleps. This requirement can impose a severe limit on the number of plant units tha may be simulated, particularly if the computer has smalt core storage. On the other hand, the modular codes witn no intermodular coupling can be used with relatively small eore storage as the commons in the cude car be small, but aecuracy may be poor unless the time intervals are very small. DYNSYS was written with partial intermodular coupling: the module equation solutions at each step of integration use coupling at all previous steps. DYNSYL, on the other hand, provides solutions with complete coupling at each present step. While developing DYNSYL, we endeavored to provide both completely coupled and uncoupled modes of sulution to provide flexibility to the user in simulating large and small plants on his computer. 


\section{UNIT MUDULE STRUCTURE}

In DYNSY L, as in any modular chemical plant dynamic simulator, the unit module provides timederivative values of the time-dependent variables (states) whenever they are needed by the integration section of the computer code. Becaluse most unit simulations are lumped parameter simulations in which values of effluent stream variables equal the vitues of these variables in the unit (well-mixed approxitistion;, only the struam variable time derivatives are computed. To have available all necessary state variable and parameter values, the unit modules must adequalely communicate those values from the main progran. In DYNSYL, as in DYNSYS, the commons rather than the subprogram arguments provide that communication. The commons contain the values of the state variables, the unit parameters, and the topology matrix. as well as other necessary values such as unil ntmber. Jigure 2 shows the makeup of the common statements in a DYNSYl. module and a short description of the code.

Aller all variable and farameter values are provided in a module subprogram, the stilte variable derivalive values inay be computed. A cer- tain amount of p:ecomputation is usually needed. particularly if the nodule allows for several options. In addition, it is often beneficial to redefine variable names in terms of the usual names for the unit operation simulated, thus helping those unfamiliur with the program to understand its function.

Figure 3 shows the typical makeup of a DYNSYL module subprogram. Optional parts are shown in parentheses. As in i)YNSYS. the module subprograms in DYNSYL are named as TYPE $n$ where $n$ is an integer. The subprograms are also identified in the executive part of the code with a descriptive name. That name should be given as a comment in the subprogram. Each subprogram should be prefaced witn a complete description of its intended i'sc, capabilities, limitations, and other information helpful to the user.

The values of the siate variables are stored in the common array $S(l . j . k)$. The array $S(2 . j . k)$ conlains the corresponding values of the sate variable derivalives. In the subprogram description presented in Fig. 1, the derivatives are lirst calculated as values of DERY (l). These values are then converted to new values in the array $S(2, j$. $\mathrm{k}$.

PROGRAI1 DYNSYL ( INPUT, OUTPUT, TAPE2 = [NPUT, TAPE3 = OUTPUT, *DATA. TAPE 4 DATA)

$L=300000 B$

CALL CREATE (4HDATA.L. ICQ)

CALL CHAHGE $(2 \mathrm{H}+\mathrm{A})$

DYHSYL

DYHSY IS AN LLL MODIFIED MERSION DF DMSYS CBARNEY, AHLLUJAL IA AND JOHNSON, FACULTY OF ENG. SCI.. UNIV. OF WESTERN OHTARID, AUGUST 1975) LAICH UTILIZES MODULAR PROGRAM I.OG IL TO SIMULATE CHEMICAL PLAHT DYNAMIC BEHAVIOR. THE TIMEWISE INTEGRATION DF THE DIFFERENTIRL EDUATIDNS GENERHTED BY EACH PROCESS UNIT MODULE IS DOHE BY THE LLL - PRDGRAM LRIVE AND ITS SUQ-PROGRAMT, A STIFF 5YSTEM INTEGRATOR LITH GREAT STABIL ITY AND ROEUSTHES5. UITH DRIVE AS THE EQUATIOH INTEGRATOR, ALL EQUATIONS GRHERATED BY THE UNIT I'DDULES ARE INTEGRATED TOGETHER.

\section{PROGRAM NOTES}

IT IS NECESSARY TO HIUMEER THE STREAMS IH THE PLANT CONSFCUTIVELY WITH THE INPUT STREAMS FIRST OH THE LIST. THIS ALLOLS THEM TO BE EXCLUDED FROM THE [NTEGRATION PROCESS. THE NUIUERING DF UHITS IN THE PLANT IS ARB ITRARY, BUT THEY SHDULD BE 1 THRU NE - UNITS, THE NLIMEER DF UNITS AND STREAMS LHICH MAY BE ACCDMODATED QY DYNSYL DEPENDS OH THE DIMENSIONING OF COMTONS AND LOCAL RECORDS. IT IS PRESEMTLY D [YTENS LONED FOR 35 UNITS AND 45 STREAITS, BUT THAT COULD BE FEAS IBLY EXPAIDED TO 45 UNITS AND 60 STREAMS TO RUN WITHIH A JOE, EOO LORD IN-CORE LIMLT. THE TIME REQUIRED FCQ A RUH UITH 69 DYMIAMIL WARIAGLES (ID STREAMS AND 6 VAR IABLES) IS TYPICALLY Q.35 MINUTES.

\section{PROGRAM DESCR LPTION}

DYNSYL IS COMFJSED OF MAIN.: INPUT PROGRAMS DYNL AND GET: A UHIT CALLIHG PROGRAM DYHZ; OUTRUT PROGRAMS OUTPIJTS, SAYEP, LVITEP.

Fig. 2. DYNSYL description and commonn blocks, (Continued on noxt page) 
LPLOT, CALPLT(DDEO PLOT): COMMTH PH:YS [CAL PROPERTY ROUTINES PROPS. TOWE, CPL I, CPVA, ENTL, LAME, DENL, DENV, VAPR, WILS, GUGL, KVAL, TEMPL, AND TEMPV; INTEGRATION ROUTINES DRIVE, STIIFF, PSET, INTERP, COSET, DEC, AND SOL: A NOISE GENERATION PROGRAI1 NOISE; AND UNIT MODEL SUEROUTINES STG IDL, CDNTLR, FIPE. PUMP, EXTRTR, PRECIP, AMD EVAPTR. THE SUBROUTIME STGIDL MAY FLASH DRUM WITH HEAT TRAMSFER, A HEAT EXCHAMGER OR A REACTOR. EACH UNIT SUBROUTINE MAY HAVE OTHER SUGROUTINES ASSOC IATED WITH IT LHICH ARE NDT NAMED HERE. EACH UHIT SUGRDUTIHE IS DESCRIBED IN MORE DETAIL WITH THE SUBROUTINE ITSELF. UNIT SUBRDUTINES ARE EASILY ADDED, AS DESCRIBED IN THE MANUAL.

THE COMMON BLOCK VARIABLES USED IN DYNSYL ARE:

\section{COMMONAMTS}

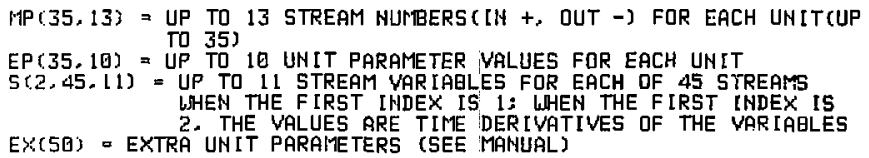

COMMOH/COH

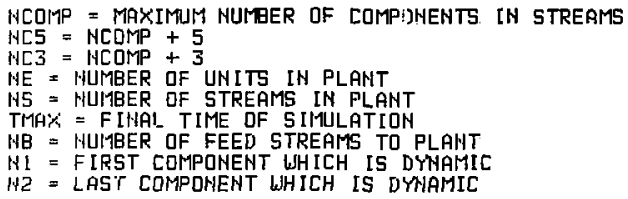

\section{COMMONAOLT,}

NOUTPT \& 1 FOR PRINTED OUTPUT AT EACH TIME IHTERYAL

NLINE I FOR A PRINT PLOT

NCAL $=1$ FOR A DDEQ PLOTCOR OTHER PLOT DEVICE)

MPR = NUMEER OF TIME IMTERVALS PER SIMULATION

NSAVE $=$ IF GT.O. POINTS UILL EE SAVED FOR PLDTTINE

HHUMO $=$ I FOR OUTPUT OF MEASURED DATA LIST (ASTATE YAR IABLES)

\section{COMMOH $/$ GEPR,}

JSTART = IF GT.D. PRRT OF SUBROUTINE SAVEP IS SKIPPED

TIME = CURREHT TIME VALUE

$H=$ IHITIAL INTEGRATOR IHTERVAL

HIHC $\approx$ TIME INEREMENT WALUE

EPS = INTEGRATION PREC [SIDN (ABSDLUTE)

\section{COMMOH GRAPHC}

NPTS = NUMGER OF PLDTTED POINT

TPLOT (150日) - TIME VALUES (UP TO 150日)

YPLOT(1500, (5) = UP TO 15 VAR IAELE VALLIES PLOTTED PER TIME VPLUE

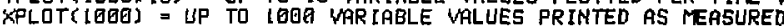
DATA PER TLME VALUE

Fig. 2. (Continuled) 


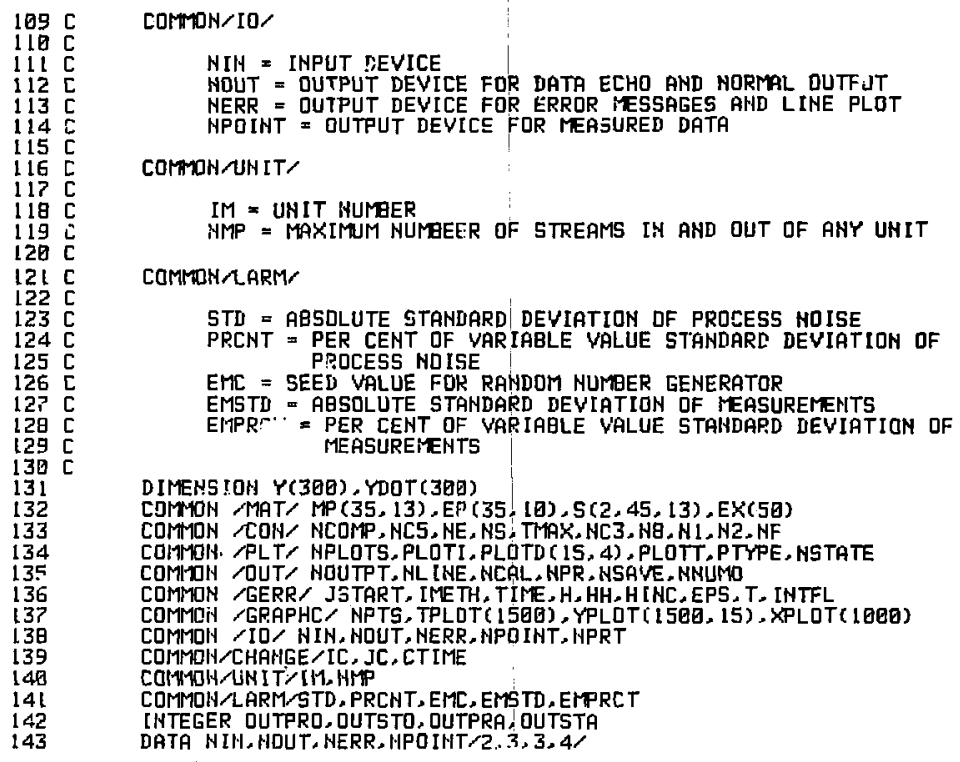

Fig. 2. (Continued) 
Subroutine Type n

(Subroutine descriptivz name)

(description of subroutine, its capabilities, limitations, and othet information helpful is users)

COMMOON/MAT/MP(35, I 3),EP(35,:0),S(2,45, I3),EX(50)

COMMON/CON, dCOMP,NCS,NE,NS,TMAX,NC3,NB,N1ST,NFIN

COMMON/GERR/JSTART,IMETH,TIME,H,HINC,EPS,TT

\section{COMMON/UNIT/MMANMP}

(REAL variable list)

(INTFGFR variable list)

(DIMIENSION variable list)

s

c (equipmest parameter list, tomporent list. cti.)

IF (INTIL., I:Q.O)* GO TO 2

RETLRN

\section{2 conTINL!}

(Rename variables Iron S-array". equipment parameters ftom EP-array, st am names from MP-array.)

(Do precompulations iectisary to compute derivatives of state variables at present timc.)

DO nn I=I, number of state variables

DERY (1) = tug*itun of state variables, equipment parameters

nn CONTINII

OTIMH:=TIMI:-HANC

INDI: $\mathrm{X}=1$

CALL DRIVI:LA,OTINI,H,Y,TIME,ESP,IMLTI,INDEX)**

(post-computations necessary)

(renanic derivative values with S-amay names)

RETLRN

IEND

Ff the subroutine talls the integrator this statement would be IF(INTFE.FQ.1)GO TO 2.

$\div S(1, j, k)$ are state variable values: $S(2, j, k)$ are their derivatives: $S(1, j, k)$ maps to $Y(i)$ for $i l_{1 e}$ integrator: $S(2, j . k)$ maps to DERY(i).

*** Jic arguments are:

$\mathbf{N}$ - number of stali varjables

OTIML - beginning time of interval

H - integration step size

$Y$ - state variable name

TIMF - Final time of interval

EPS - convergence criterion (fraction of variable)

METH - integration mediod (see integrator manual ${ }^{14}$ )

Fĭg. 3. I nit module subprogran description. 


\section{THE INTEGRATOR}

The integrator used in DYNSYS is a modification of the original GEAR 12,13 integration code for stiff systems of differential equations. It is a subroutine called by each unit niodule subprogram to integrate the differential equations represented by the differentials of the state variables. In DYNSYS the integrator is a two-step predictor-corrector with a maximum order of six. The integration is done either by an Adam-Bushforth method requiring storage of previous derivative values, or by a "stiff" method requiring storage of previous state variable values. The integrator stores the values of all differential or siate variables up to the order six from all the unit module subprograms. For the present time interval, all stute variable values determined by the predietor step are available for use in the corrector step. Therefore, the previous state variable values account for coupling between equations, which represents all units in the simulated plant.

Besides including intermodular coupling, the GEAR-based integrator in DYNSYS is designed to handle stiff sets of equations, those with a wide range of time constunts in the various differential equations integrated. Unfortunately, use of DYNSYS with the unit module STGIDL (see Appendix $A$ ) caused many unstable cases that were impossibie to integrate. For that reason an improved integration subprogran was sought for DYNSYL.

The subprogram chosen for DYNSYL is a version of the GEAR stiff integrator written for use at LLL. ${ }^{14}$ It was designed to serve as the integrator in locally generated, continuous-system simulations. Its main program. DRIVE, is called by the simulator. DRIVE then calls STIFF which calls a user-written subprogram to generate values of the differentials to be integrated. When used with
Outputs, WRITEP, CALPLT OUTPUT Input values, values of state, variables and equation parameters at each time, graphic output
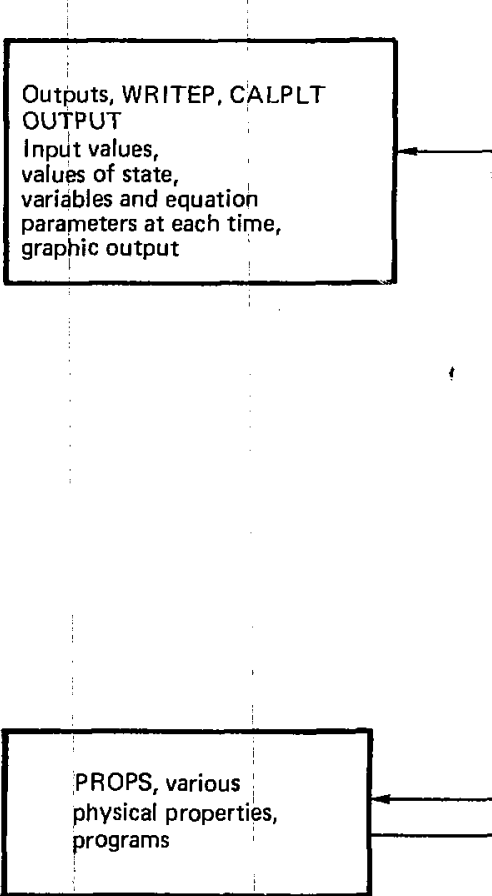

ligh. 4. Coupled mode of uperation of DYNSYI. (generul siructure.

DYN 1, GET Input initial values, equation parameters, calculation parameters, defaults, physical parameters, property coefficients

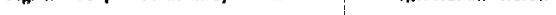


DYNSYL, the LLL version of GEAR is called by the main pregram of DYNSYL. GEAR then calls for values of each of the differentials in the unit modules through an ordering program called DYN2 (see 1Fig. 4). Under these circumstances the integrator uses present values of all differentials at each integration step.

An alternate use of GEAR in DYNSYL is designed to use less memory capacity. In that mode the integrator is called by each individual unit module subprogram. The conimons are then only as large as the largest subprogram common because all stale variable values for all orders are nol stored. The alternate mode ilceounts for intermodular coupling only through the state vatiable talues from the last time step.

To efficiently solve large sets of differential equations, the GEAR integrator can use a NewtonRaphson method requiring Jacoblan values for the state variables. The integrator manual itexplains all the options for its use, but for the purposes of DYNSYL we only considered iwo: analytical computation of the Jacobian values in the unit module subprograms, and numerical computation of the Jacobian values by the integrator. The former choice saves computer time but the batter is inore flexible, as analytical Jacobian expressions can, in many cases. be almost impossible to derive. So far. we have only used the numericat option.

\section{OVERALL PROGRAM STRUCTURE}

The gentral strutere of DYSSYL is shown in lig. 4. The main program first calls al dati-reading program. DYN1. which also use GFT. which, in turn. primarily reads alphanteric information. The main program calls the integrator program DRIVE. (after all necessilns precomputations hase been mude): ORIVl citl DYN2. the unt moduic program culler. through $571 \% \%$ a subprogram of I)RIVt:. Io determine undated values of the state variables alter eilch tame increment. the unil module program compute salues of the derivatives of the state variables th the uned by the integrator program. The unit module programs call the physical property program when necessary. Al the end of each oulput interval several integrator time intervals), the integrator returns to the main program so that the output programs maly be called.
The strueture shown in Fig. 4 is for complete coupling of all equations of all units. If it is desired to save memory snace in the computer, or if a vers large chentical plant is 10 be simulated, an uncoupled mode maty be used. The structure of that mode is shoun in ligh. 5

The uncoupled mode is mo kearly a ellicient as the coupled mode. nor is it at accurat s. beciasse the integrator must be initialised at the bexintumg of eich time step of MAIN if the integrator is being talled by more thatn one unit module. The multipleorder capabilities of the integratur a de also interrupled at eitch time step of $11 \mathrm{M}$.

The logic used in ellter the coupled or uncoupled mode is shoun in lik. b, wheh shoss the detailed organtation of ly bist primarly throigh subroutine calls

\section{PROCESS TOPOLOGY AND PROCESS VARIABLES}

To transmit to the unit module subprograms those process streams connected to then. it is necessary to define a process topology arra!. MP (i.j), whose values are the numbers of the streams defined within the unit modules. $A$ unit module mi ht. for instance. have three streans connected to it 1.umbered 1. 12, and 33. If the unit in the plant is nulaber 2. then the topology matrix values would be $M P(2.1)=1 . \quad M P(2.2)=12$. and $\operatorname{MP}(2.3)=3.3$. Such an array makes possible greater flexibility than if the stream numbers are assigned wjihin the unit modules during each use of the simulation code.
The values of the process sarbables are conn. tained in the arrat Sti.t.h Is mentioned previousis. when $1=1$. the vallew are the state variable values; when $i=$ ?. the bluev are the derivatizes computed in the unit modules. The salue of $j$ is the stream number. The salues of $k$ indieate the particular stream variables an follow as 3 for flow ratte. 4 for temperature. 5 lor pressure. and o to n for concentrations of various components. where $n$ is the maximum dimeition of $k$. The value of the siream number is $S(1, j, 1)$ and $S(1, j, 2)$ is a flag whose value maly be positive or negative. If the stream flag 


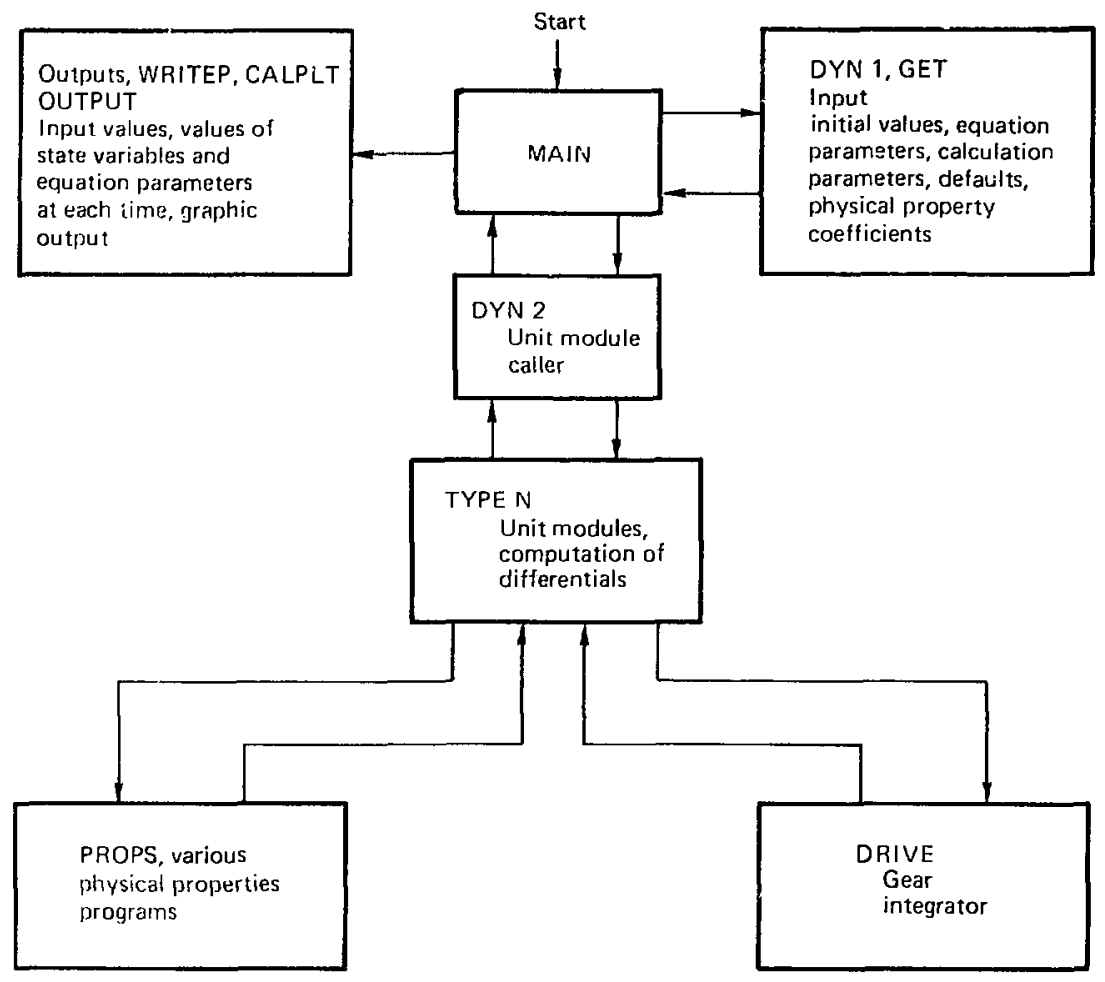

liz. 5. I ncoupled nade of operation of DY VShI. (general structureI.

is negatict, that stream vecter is suppressed in the outpul.

l:quipmenl and other parameter vilues are contained in the array $E: P(i, j)$. where $i$ is unit num- her and $\mathrm{j}$ is parameler number. $l$ :ach unit mat have up to 10 parameters in that array. plus additional , nes in vector $\mathrm{I} X(\mathrm{i})$. Thall veclor will be discussed lurther in the stetion on IXPlit.

\section{VARIABLES AND ARRAYS}

Tht process topology, variable, and equipment parameter arrays are the main common arrays usted in DYNSYL. Many other arrays and common variables are defined in the listing from the main
DYNSYL program shown in Fig. 6. Most of thest are self-explanatory or become clear with use of the DYNSYL code, and thus we will not describe them hers. 


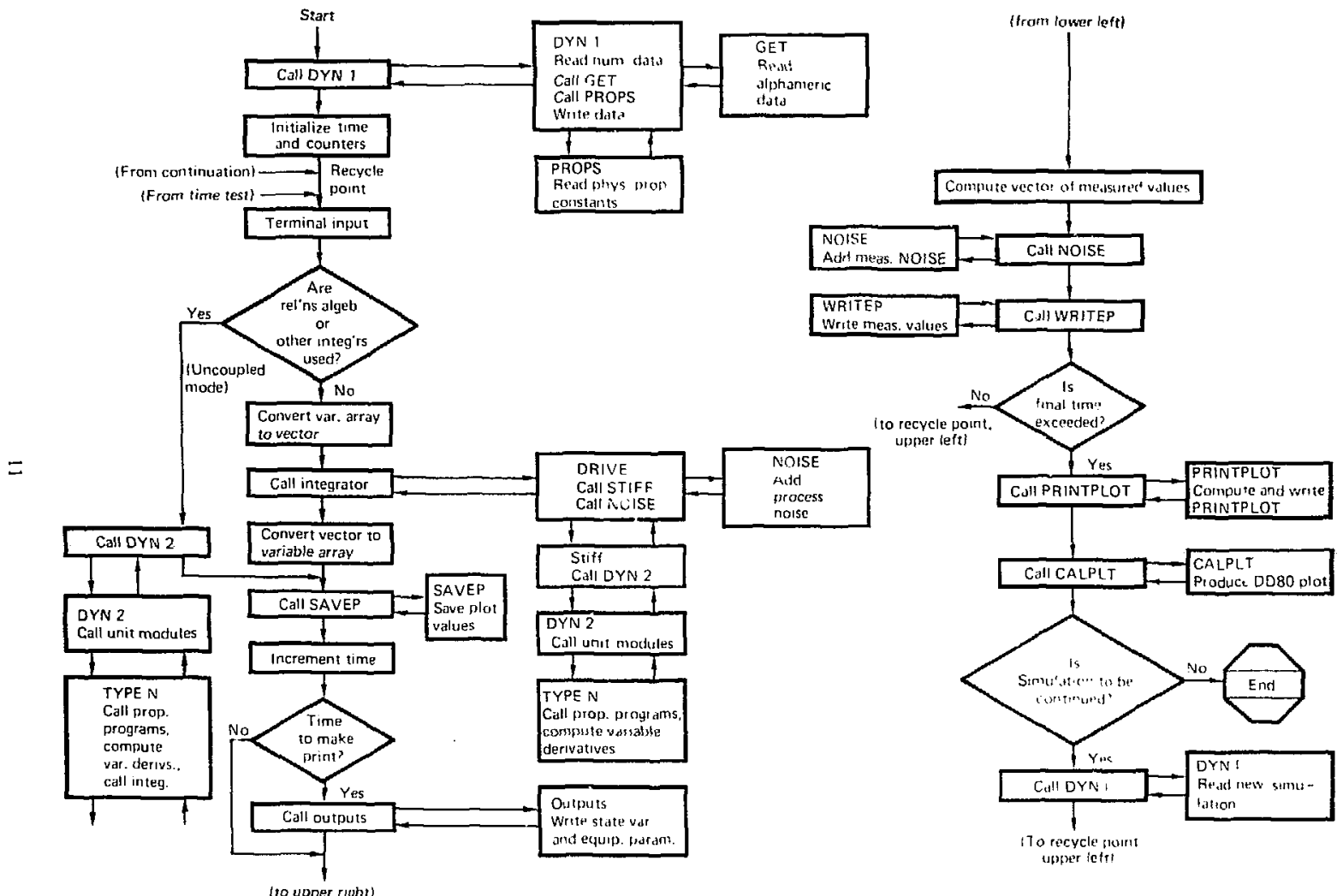

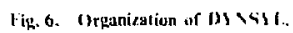




\section{ERROR MESSAGES}

The error messages generated by DYNSYL are a conbinateon of those from the original DYNSYS and from the (il:AR-inlegrator code. The messatges are usually elear, but the proper remedy masy not be. The IJY XSYS manual' and the integrator marrual th contain suggested responses to tach of tise error messages that may be generated by the code.

\section{PROGRAM EXECUTION}

Ipon execulion of the DYNSYI. code, the coded inpul values are firss read by subroutines DYNI and (il:). The user is then immediately informed of the liMli vilute and asked for the TIMI: value for the first change-in-stute variables. Such changes may be made to simulate process upsets, material diversions, or control actions. The requested TIMP value is input through the terminal. and execuljon resumes.

The user in informed when it is time for a change, and is asked to type the number of state virriables $t o$ be changed. He is then asked for the strean and variable numbers as well at the new viriable value for each thange. The proper format for the inpul is alwayn given. After all changes are given, the user is asked for the next TIMt: value for which a change will be made. Whenever that IIMI: value exceeds the simulation time execution is completed.

The files containing nunterical and eriphoual output are crealed when the execution is completed. The outpul deviees from which thene files maly be obtained by the user depend on computer inpe and user specifications.

\section{JNPUT}

The form of the input specifications for DYNSYl is mearly the same as in DYNSYS. (For a very complete description of the input system used, the DYNSYS manual I may also be consulted.) To ilIustrate the input format and some of the variations, the example input shown js, ligg. 7 will be discussed below. The example is for simulation of a 14-stage extritctor, which is shown schematically in Fig. 8.

One may analyze the necessary format for the input dita hy reviewing DYNl, given in the listing in Appendix $B$. There are several default values that are first established. These are listed in Table $I$.

To override the default values, input values may be read as shown in Table 2 [FORMAT (3A4. F(2.5)].

A new unit module not already included as part of the module library can be inserted into the code through the input data. The alphameric LIBRARY signals new module-nume input as shown in the example in Fig. 8. The number uffer LIBRARY is the number of new modules. The next lines are the new module names and their numbers. Note that all numerical input is floating-point even when the numbers are used as islegers. The formats are all (3^4, F12.5).

The alphameric PROCESS signals the biginning of equipment and process data. An input listing is required. consisting of at unit module name and the unit number followed by the steam numbers (in and out of the unit) and equipment parameters in the proper order (specified by the unit module subprogramy. If unit numbers are negatlive. equipment paranelers will be output. If stream numbers are negative, stream flows are outward. The formals are $(3 \mathrm{~A}+, 1-12.5)$ for unit name and number and $(12 \mathrm{X} .51 \% 2.5)$ for the stream numbers and equipment paramelers.

After the ilphameric 1 is). the number of 5:. wams and stream variable values at sere time are iripul. The stream variable values should be given in the order indicated for the state variable array discussed previously, with the stream number and flatg as the first two. If the stream flag is negative, output is suppressed. Again the format is $(12 \mathrm{X}, 5 \mathrm{~F} 12.5)$. The alphameric EXPL.ICIT or SPECIAl. comes before euch strum sariable list. FXPLICIT indicates that values for il particular stream are listed. SPFCIAL followed by a stream number indicates that values for that stream number through the stream number indicaled in the array list are given. SPECIAL allows input of common values for a number of streams using one input list.

After the alphameric END. PROPERTIES indicates that coefficients for several different 
proper!s correlatom equatiuns for tach component will be read. The number of property tyuations depends on the number following PROPERTIES. If -1 or -2 follows PROPERTIES, property coefficients for waler or alr, respectively, ars assumed by the program. The property equation coefficient input in DYNSYI. is the same as in DYNSYS: sete Rel. I for details. The formdts are (3A4. F12.5) and (12X. it 12.5).

a fier another alphameric IEND, GRAPH indicates with a ubseyuent number several graple lines (nariablest to he ploted by (AL_PI.T or I.Plot. The tho numbers on the next line indicale the abscossit inerement and the lineplot wide. If the second number is 1,0 , the lineplot is 50 spaces wide: if it is 0.0 . the lintplot is 100 spaces wide. The next ines give the specifications for tach variable graphed. the stream number and variable. and the lower and upper ordinate limits.

If the user desires to continue with a different number of iterations in a given time, or with a different number of iterations between printing. he follows the procedure that we showed in the example. The eme given is the final time, and the number of iterations is the number as if beginning at zero time. The prompt END must be given after each antinuation specification and also to end the simulation.

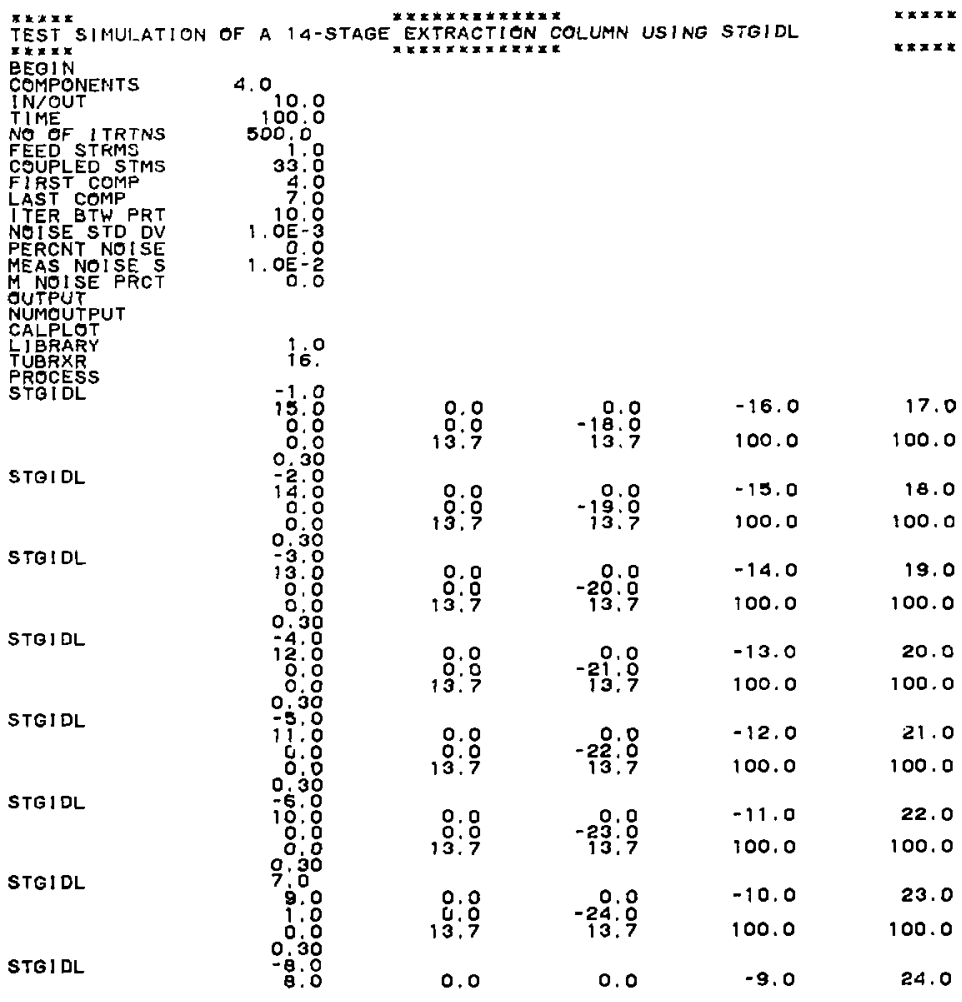

Fip. 7. Example input for a 14-stuge extractor with diversion on input line. (Continued on next page) 


\begin{tabular}{|c|c|c|c|c|c|}
\hline \multirow{3}{*}{ STOIOL } & \multirow{3}{*}{$\begin{array}{r}0.0 \\
0.0 \\
0.30 \\
-9.0 \\
7.0 \\
0.0 \\
0.0 \\
0.30\end{array}$} & \multirow{3}{*}{$\begin{array}{r}0.0 \\
13.7 \\
0.0 \\
0: 0 \\
13.7\end{array}$} & \multirow{3}{*}{$\begin{array}{r}-25.0 \\
13.7 \\
-29.8 \\
-26.9\end{array}$} & \multirow{3}{*}{$\begin{array}{r}100.0 \\
-0.0 \\
100.0\end{array}$} & \multirow{3}{*}{$\begin{array}{r}100.0 \\
25.0 \\
100.0\end{array}$} \\
\hline & & & & & \\
\hline & & & & & \\
\hline STOIOL & $\begin{array}{r}-10.0 \\
6.0\end{array}$ & 0.0 & 20.0 & -7.0 & 26.0 \\
\hline & 0.30 & 13.7 & 13.7 & 100.0 & 100.0 \\
\hline STGIOL & $\begin{array}{rl}-11 & 1 \\
5 & : 0 \\
0 & 0\end{array}$ & O. & -30.0 & -6.0 & 27.0 \\
\hline & 0.30 & 13.7 & 13.7 & 100.0 & 100.0 \\
\hline STOIOL & $\begin{array}{r}-12.0 \\
4: 0 \\
0: 0\end{array}$ & 0.8 & $\begin{array}{r}0.0 \\
-25: 0\end{array}$ & $-5,0$ & 28.0 \\
\hline & 0.00 & 13.7 & 13.7 & 100.0 & 100.0 \\
\hline STOIDL & $\begin{array}{r}-13: 0 \\
3: 0 \\
0: 0\end{array}$ & 0.0 & $-30: 0$ & -4.0 & 29.0 \\
\hline & 0.30 & 13.7 & 13.7 & 100.0 & 100.0 \\
\hline STOIDL & $\begin{array}{r}-14.0 \\
2 .: 0 \\
0.0\end{array}$ & $\begin{array}{l}0.0 \\
0.0\end{array}$ & -3i:o & -3.0 & 30.0 \\
\hline & 0.30 & 13.7 & 13.7 & 100.0 & 100.0 \\
\hline STGIDL & $\begin{array}{r}-15.0 \\
32.0 \\
0.0 \\
0.0 \\
0.0\end{array}$ & $\begin{array}{l}\text { g.: } \\
\text { a:o } \\
0 .: 1\end{array}$ & $\begin{array}{rl}r 1 & 0 \\
0 & 0 \\
0.0\end{array}$ & $\begin{array}{r}-33.0 \\
0.0 \\
0.0\end{array}$ & $\begin{array}{l}0.0 \\
0: 0 \\
0.0\end{array}$ \\
\hline 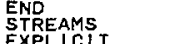 & 33.0 & & & & \\
\hline EATLIOI & $9 \cdot 0$ & 4.78 & 7.28 & $\begin{array}{r}25.0 \\
0.0\end{array}$ & 1.0 \\
\hline GTCUL & 16.0 & 1.0 & 2.43 & 25.0 & 1.0 \\
\hline SPEC IAL & $\begin{array}{l}17.0 \\
23.0\end{array}$ & 1.0 & 1.02 & 25.0 & 1.0 \\
\hline SPECIAL & $\begin{array}{l}24.0 \\
31.0\end{array}$ & 1.0 & 8. 3 & 25.0 & 1.0 \\
\hline EXPLIC1T & $\begin{array}{r}32.0 \\
9.5\end{array}$ & 4.78 & $\begin{array}{r}7.28 \\
3.1\end{array}$ & 25.8 & 1.0 \\
\hline EXPLICIT & 33.0 & 1.0 & 0.0 & 25.0 & 1.0 \\
\hline $\begin{array}{l}\text { END } \\
\text { PROPERTIES }\end{array}$ & -1.0 & & & & \\
\hline $\begin{array}{l}\text { END } \\
\text { GRAPH }\end{array}$ & $\begin{array}{r}4.0 \\
2.0 \\
16.0 \\
31: 0 \\
190\end{array}$ & $\begin{array}{rl}0.0 \\
7 & 0 \\
7 & 0 \\
3 & 0 \\
7.0\end{array}$ & $\begin{array}{l}0.0 \\
0: 0 \\
0: 0 \\
0.0\end{array}$ & $\begin{array}{l}20.0 \\
1.00 \\
10.0 \\
10.0\end{array}$ & \\
\hline $\begin{array}{l}\text { TIME } \\
\text { NO OF I TRTNS } \\
\text { ITER B TW PRT } \\
\text { END } \\
\text { CONTINUE } \\
\text { TIME NU I TRTNS } \\
\text { NO OG ITER BTW PRT } \\
\text { ITER BTW } \\
\text { END } \\
\text { END }\end{array}$ & $\begin{array}{r}1000 . \\
100 \\
2: \\
1000 \\
1000.0 \\
500.0 \\
10.0\end{array}$ & & & & \\
\hline
\end{tabular}

Fig. 7. (continued) 


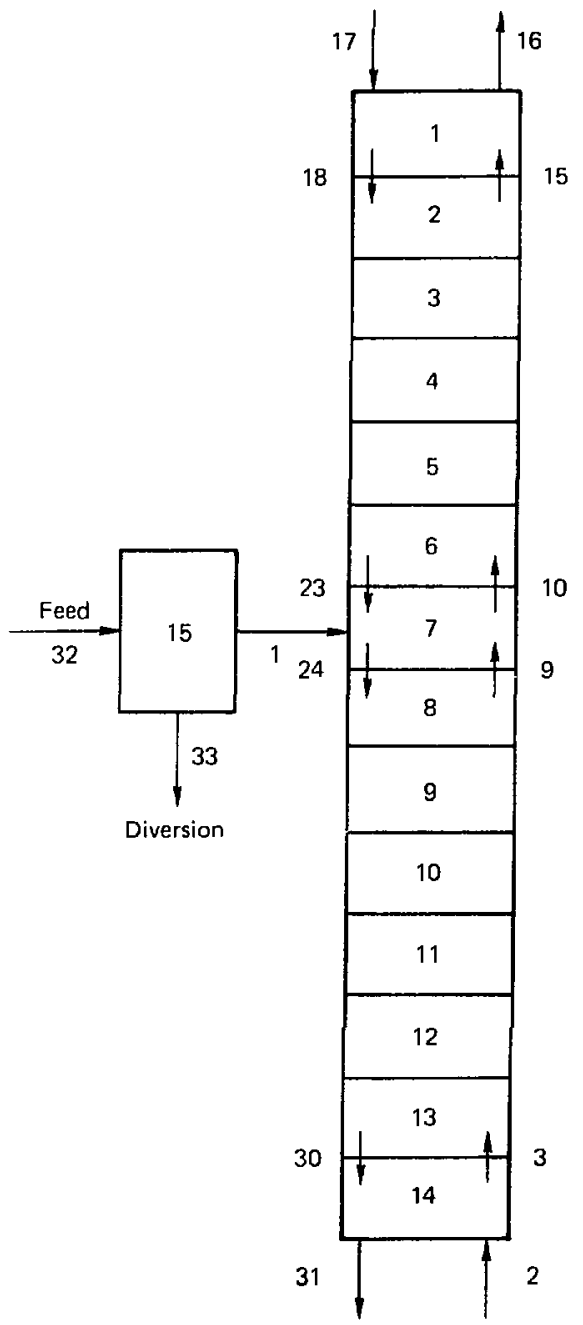

Fig. 8. Diagram of example extraction column. I'nit 15 is of nearly zero colume for diversion simulation. 
Table 1. Input defoult values.

\begin{tabular}{|c|c|}
\hline Input varialsles & Derijnition \\
\hline $\operatorname{EMC}=0.0$ & Argument for random number generatur \\
\hline NIST $=1$ & First variable ta lue a state vartahle \\
\hline $\mathrm{NIF}=6$ & L.ast variable lo be a state variable \\
\hline$N B=1$ & Number of menvariable streams \\
\hline $\mathrm{MAXNE:}=35$ & Maximum number of unit \\
\hline TIMI: $=0.0$ & Initial time valut \\
\hline ISTIIFF=2 & Stiff method of integration: I for Adatas . Yethod \\
\hline MrSJ:H $=2$ & Numerically computed Jacolsian: see JKIVI: in listing \\
\hline $\mathrm{NCOMP}=1$ & Number of components \\
\hline$N M I^{2}=5$ & Maximum number of stretams in and ant l of a unit \\
\hline $\mathrm{H}=\mathbf{1} \cdot \mathbf{8}$ & Initial time increment \\
\hline$T H A X=10.0$ & Simulation time \\
\hline$N P R=1$ & Number of iterations to be run \\
\hline $1: P S=0.001$ & Convergence criteriun \\
\hline NOUTPT $=0$ & No printout of state variable values \\
\hline NNUSOO-0 & Non printerut of measurement vectors valmes \\
\hline NI.INE: $=0$ & No linejplot \\
\hline$N C A L=0$ & No DD80 plot \\
\hline NOMISS $=0$ & No flonfatal error messagch \\
\hline $\mathrm{STD}=0.0$ & Standard deviation of process moise \\
\hline PRC.NT=0.0 & STU as pereent of state variable value \\
\hline EMSTD $=0.0$ & Standard deviation of measurement mise \\
\hline FMPRCT $=0.0$ & EMSTD as percent af metsured variable value \\
\hline NRPI $=1$ & Number of iterations between printing. \\
\hline
\end{tabular}


Table 2. Input values.

\begin{tabular}{|c|c|c|}
\hline Input variabla & Numerical valase & Definition \\
\hline UIGIN & & Slarts input process: all preceeding lobels are ignored \\
\hline NONSTHFI: & & Causes nunstifr integration \\
\hline COMPONENTS & & Nutnber of components \\
\hline INOT1 & & Number of streams in and out of unit \\
\hline H:LD STRI-AMS & & Number of nonvariable streams \\
\hline FIRSI VAK & $x$ & First variable to be a state variable \\
\hline LASI VAK & $\mathbf{x}$ & Last varialıle to be a state varialsle \\
\hline DI.J & $x$ & Initial time inctement \\
\hline Tist: & $x$ & Simulation time \\
\hline No $O F \cdot I T H K$ & $x$ & Number of interations to be run \\
\hline TOLH:RANCI & $x$ & Convergence criterion \\
\hline ITER HTW J'RI & $x$ & Nember of iterations between printing \\
\hline Noisl- & $x$ & Standard deviation of process noisc \\
\hline M:RCLEI XOIS & $x$ & Above as percent of state variable value \\
\hline MI:AS NoISR: & $x$ & Standare deviation of measurement noise \\
\hline A NOISF JRCJ & $x$ & Ahuve as percent of state variable value \\
\hline OLTPLI & $\mathbf{x}$ & Print state variable values \\
\hline 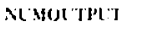 & $x$ & Print measurement vecter values \\
\hline I.INI:L'ILI'T & $x$ & Print plot to be output \\
\hline CALPIOI & & DD80 plost to be produced \\
\hline No.11!SSAC;! & & 'rint nonfatal errur messiges \\
\hline IATI:G; M:TIIOD & $\dot{x}$ & Value of MlTEK in integrator. \\
\hline
\end{tabular}

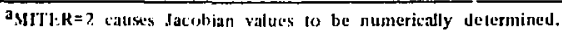




\section{OUTPLT}

The oulput of ISYNSYl. begins with an echo of the input in almost the same form, as shown in Fig. 9. At each print time beginning with IIMI: $=0.0$, all stream variables except for suppressed streams fogative stream number in STRI:AMS input) are prinled. Figure 9 show a sampling of output at various times. lor each negitive unit number the equipmem specilications are also geven at each print time. Any changes in stream varables or equipment parameters by interactive inpul are, of courst. rellected in the output at the next print ume.

If a lineplot is specified, it uill follow the state variable and equipment parameler output after tach simulation time omilad time and eaclo continuations. Some cilre must be exeresed in specifying the tims. fabscissa) spacing. as the lineplot can become excessivedy long.
The I)DXo nlot produced by (MI.PI.I is plated on disk under the name PI IX A separate plot is produced for each contunuation of the simulation. That form of us:put is frequentis the most useful for comparisons of sarious run condi. tions. I kitmples for the input and eutput are shoun in Jigs. 10 through 12.

Anotiser form of output produced by DYXSYI. is tailored for online data analysis methods such as Kaimin filters. I he meisurement datat vec. tor is computed in the MAlN program. The vector may conlatin any consecutive lise of variables from catsts stream that is not an inpul (constant) stream. Vilucs from each interaction of $M 1 \mathrm{AIN}$ are printed

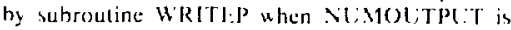
specified in the inpul. The form of the output is simply the tume value followed by each state variable value in order with a format of (6) 12.3$)$.

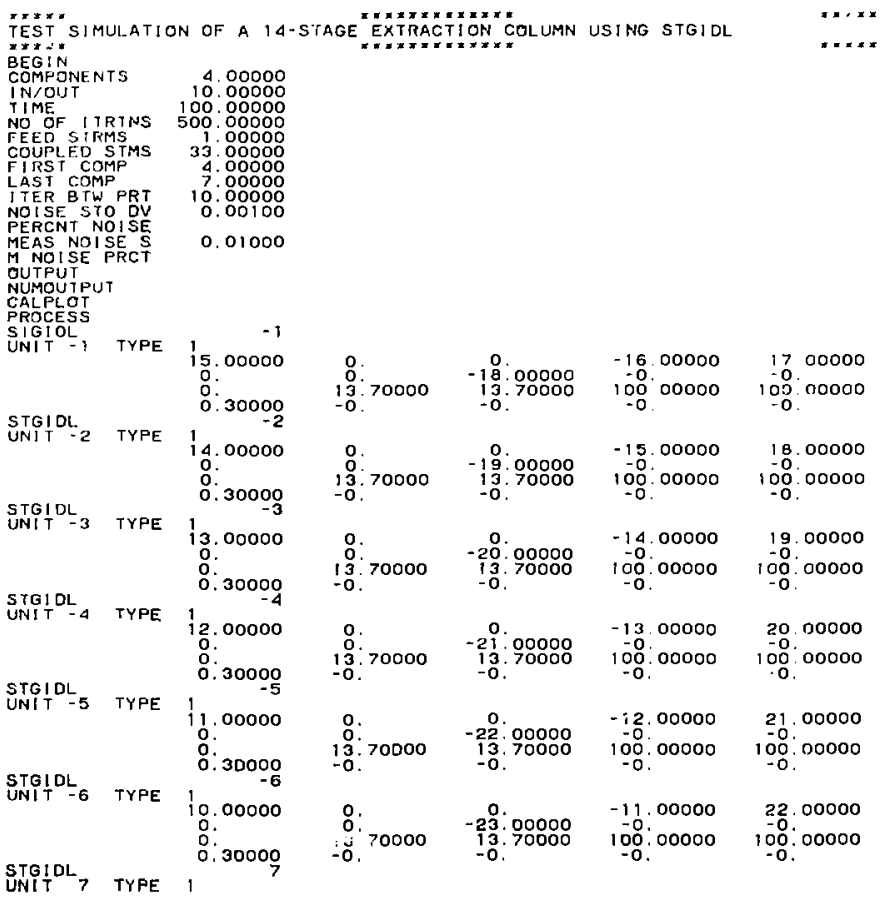

Fig. 9. Jixumple output for \& 1+stage exiractor with divertor on input line. (Continued on next puge) 


\begin{tabular}{|c|c|c|c|c|c|c|}
\hline & & $\begin{array}{l}9.00000 \\
1.00000 \\
0.30000 \\
0.300\end{array}$ & $\begin{array}{l}0 . \\
\text { o. } \\
13 \\
13 \\
-0\end{array}$ & $\begin{array}{r}0.00000 \\
-24.00000 \\
13.70000 \\
-0 .\end{array}$ & $\begin{array}{r}-10,00000 \\
-0.00000 \\
100.0000 \\
-0 .\end{array}$ & $\begin{array}{r}23,00000 \\
-0,00000 \\
100,00000 \\
-0,\end{array}$ \\
\hline STGIDL & TYPE & $\begin{array}{l}1 \\
8.00000 \\
0: \\
0: 30000 \\
0.9\end{array}$ & $\begin{array}{l}0 . \\
0 . \\
13 \\
1.70000 \\
-0\end{array}$ & $\begin{array}{l}0 . \\
-25,000 n n \\
13,70000 \\
-0 .\end{array}$ & $\begin{array}{l}-9,00000 \\
-0.0000 \\
100.00000 \\
-0 .\end{array}$ & $\begin{array}{r}24.00000 \\
-0.00000 \\
100.00000 \\
-0 .\end{array}$ \\
\hline UNIT' & TYPE & $\begin{array}{l}1.00000 \\
7.9 \\
0: \\
0.30000 \\
0.10\end{array}$ & $\begin{array}{l}0 . \\
0 . \\
13.70000 \\
-0.7\end{array}$ & $\begin{array}{l}0 . \\
-26 \\
13 \\
-00000 \\
-0.700 C 0\end{array}$ & $\begin{array}{l}-8,00000 \\
-0.00000 \\
100 \\
-0 .\end{array}$ & $\begin{array}{r}25.00000 \\
-0.00000 \\
100: 00000 \\
-0 .\end{array}$ \\
\hline STGIDL, & TrPE & $\begin{array}{l}1 \text { 6. } 00000 \\
\text { o. } \\
0 . \\
0.30000 \\
-11\end{array}$ & $\begin{array}{l}0 . \\
0 . \\
13.70000 \\
-0.7\end{array}$ & $\begin{array}{l}0 . \\
-27,00000 \\
13,70000 \\
-0\end{array}$ & $\begin{array}{l}-7.00000 \\
-0.0000 \\
100.00000 \\
-0.0\end{array}$ & $\begin{array}{r}26.00000 \\
-0.00000 \\
100.00000 \\
-0 .\end{array}$ \\
\hline $\begin{array}{l}\text { STGIDL, } \\
\text { UNIT: }\end{array}$ & TYPE & $\begin{array}{l}1.00000 \\
0.000 \\
0.30000 \\
0.30\end{array}$ & $\begin{array}{l}0 . \\
0.7 \\
13.70000 \\
-0 .\end{array}$ & $\begin{array}{r}0.0000 \\
-28.00000 \\
13.70000 \\
-0 .\end{array}$ & $\begin{array}{r}-6.00000 \\
-0.00000 \\
100 \\
-0.00000\end{array}$ & $\begin{array}{r}27.00000 \\
-0.000000 \\
100.00000 \\
-0 .\end{array}$ \\
\hline $\begin{array}{l}\text { STGIDL } \\
\text { Ul.1 }\end{array}$ & $\because \forall \beta E$ & $\begin{array}{l}1.12 \\
4.00000 \\
0.0 \\
0.30000 \\
0.13\end{array}$ & $\begin{array}{l}0 . \\
0 \\
3 \\
-3 \\
-3\end{array}, 70000$ & $\begin{array}{l}0 . \\
-29.70000 \\
13.70000 \\
-0 .\end{array}$ & $\begin{array}{r}-5.00000 \\
-0.00000 \\
100.0000 \\
-0.0\end{array}$ & $\begin{array}{r}28.00000 \\
-0.00000 \\
100.00000 \\
-0 .\end{array}$ \\
\hline Untio! & TrFP & $\begin{array}{l}3.13 \\
3.00000 \\
0 . \\
0.30000 \\
0.14\end{array}$ & $\begin{array}{l}0 . \\
0 . \\
13 \\
0 .\end{array}$ & $\begin{array}{l}-0 . \\
-30.70000 \\
13.70000 \\
-0 .\end{array}$ & $\begin{array}{r}-4.00000 \\
-0.00000 \\
100.0000 \\
-0 .\end{array}$ & $\begin{array}{r}29.00000 \\
-0.00000 \\
100.0000 \\
-0 .\end{array}$ \\
\hline DNA & $\therefore P E$ & $\begin{array}{l}1,14 \\
2 \\
0 \\
0 \\
0 \\
0\end{array}$ & $\begin{array}{l}0 . \\
0 \\
13 \\
13 \\
0 .\end{array}$ & $\begin{array}{r}0 . \\
-31 \cdot 00000 \\
13: 70000 \\
-0 .\end{array}$ & $\begin{array}{rl}-3 & 00000 \\
-0 & 000 \\
100.0000 \\
-0 .\end{array}$ & $\begin{array}{r}30.00000 \\
-0.00000 \\
100.00000\end{array}$ \\
\hline $\begin{array}{l}\text { STGIOL, } \\
\text { INNIT: }\end{array}$ & Trpit & $\begin{array}{ll}1 & -15 \\
32 & 00000 \\
0 & \\
\mathrm{a} & \\
\mathrm{a} & \end{array}$ & $\begin{array}{ll}0 & \\
0 \\
0 \\
o \\
0 \\
0 & 10000\end{array}$ & $\begin{array}{l}-\dot{a}, 00000 \\
o \\
o \\
-0\end{array}$ & $\begin{array}{c}-33.00000 \\
0 \\
-0\end{array}$ & $\begin{array}{l}0 . \\
: \\
0:\end{array}$ \\
\hline $\begin{array}{l}\text { END } \\
\text { SFREAMS } \\
\text { EXPLIT, } \\
\text { SPECIAL } \\
\text { SPECIAL } \\
\text { SPECIAL } \\
\text { EXPLICIT } \\
\text { EXPLICII }\end{array}$ & & 33 & & & & \\
\hline $\begin{array}{l}\text { END } \\
\text { STRE AM }\end{array}$ & & 1.00000 & 1.0000 & 7280 & 2500000 & 1.00000 \\
\hline STREAM & & $\begin{array}{r}9.50000 \\
2.00000 \\
-0 .\end{array}$ & $\begin{array}{rr}4780000 \\
-1 \\
-0\end{array}$ & $\begin{array}{r}\text { 3. } 10000 \\
\text { 2. } 43000\end{array}$ & - $5^{\circ} 00000$ & 1.00000 \\
\hline STREAM & & 3 anaoo & -0.00000 & 2. 43000 & 2500000 & 100000 \\
\hline STREAM & & 4.00000 & 1.00000 & - $2: 43000$ & 25.00000 & 1.00000 \\
\hline STREAM & & 5,00000 & -0.00000 & ${ }^{-0} \cdot 43000$ & 2500000 & 1.0000 \\
\hline STRERM & & $\begin{array}{c}-0 \\
-0 \\
-0\end{array}, 00000$ & 1.00000 & $\begin{array}{l}-0 \\
2\end{array} 43000$ & 25.00000 & 1.00000 \\
\hline STREAM & & 7.00000 & 1.00000 & -a. 43000 & 2500000 & 1.00000 \\
\hline STREAM & & $\begin{array}{r}-0,00000 \\
-0,0\end{array}$ & $\begin{array}{l}-0.00000 \\
-0.000\end{array}$ & - & $\begin{array}{l}-0 \\
25\end{array} 00000$ & 1.00000 \\
\hline STREAM & & 9,00000 & 1.00000 & $\frac{2}{2}: 43000$ & 2500000 & 1. 00000 \\
\hline STREAM & & $10: 00000$ & ${ }_{-0}^{1}: 00000$ & $-4: 43000$ & 25,00000 & 100000 \\
\hline STREAM & & 11.00000 & 1.00000 & $2 \cdot 43000$ & 2500000 & 1.00000 \\
\hline STREAM & & $\begin{array}{l}-0.00000 \\
12.000\end{array}$ & -0.00000 & $-4: 43000$ & 2500000 & 1.00000 \\
\hline STREAM & & 13.00000 & 1.00000 & 43000 & 25.00000 & 1.00000 \\
\hline STREAM & & 14.00000 & 1. oaooa & -0.03000 & 25.00000 & 1.00000 \\
\hline STREAM & & 15.00000 & 1.00000 & $\begin{array}{l}0.0 \\
2\end{array} 43000$ & 2500000 & 1.00000 \\
\hline STREAM & & $16: 00000$ & $\begin{array}{l}1: 00000 \\
-0 .\end{array}$ & $\begin{array}{l}2.43000 \\
-0.4\end{array}$ & $\frac{25}{-0}: 00000$ & 1.00000 \\
\hline
\end{tabular}

17ig. 9. (Continued) 


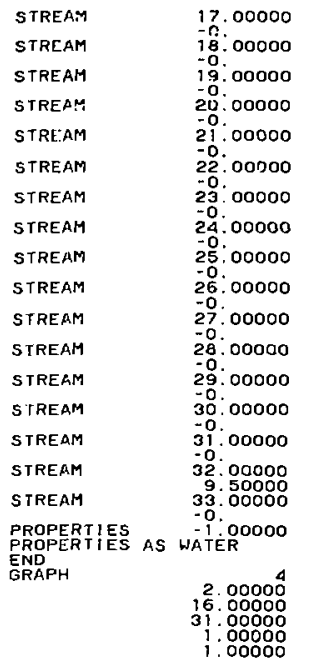
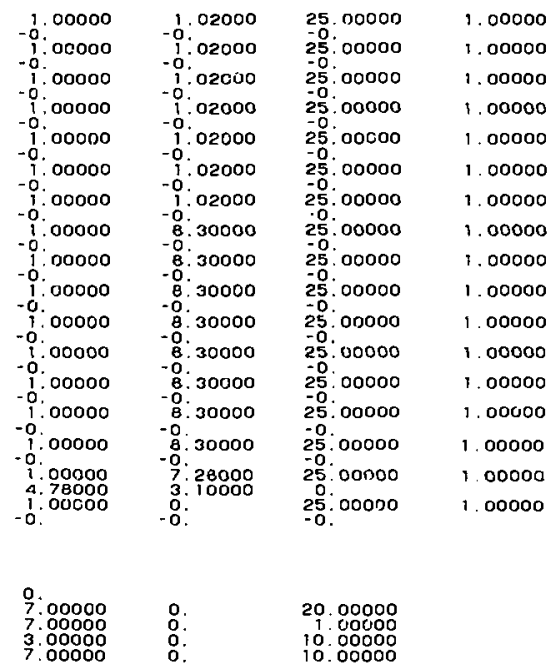

20.00000
1.00000
10.00000
10.00000

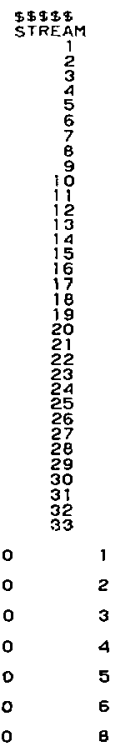

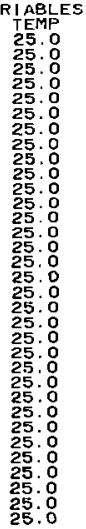

0 .

0.

0 .

o.

o.

0 .

0 .

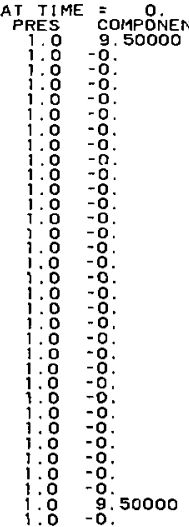

13.70000

13. 70000

13, 70000

13. 70000

13. 70000

13. 70000

13.70000

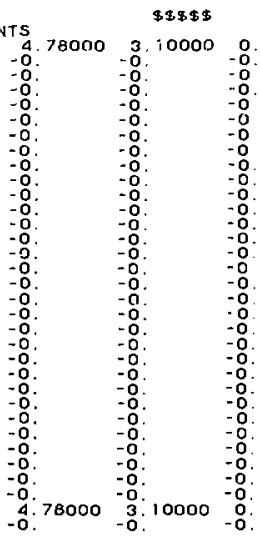
13. 70000
13. 70000
13. 70000
13. 70000
13. 70000
13. 70000
13. 70000

100.00000

100,00000

100.00000

100.00000

100.00000

100.00000

100.00000
100.00000

100.00000

100.00000

100.00000

100.00000

100.00000

100.00000

Fig. 9. (Continued) 


$\begin{array}{ll}0 & 9 \\ 0 & 10 \\ 0 & 11 \\ 0 & 12 \\ 0 & 13 \\ 0 & 14 \\ 0 & 15\end{array}$

13.70000
13.70000
13.70000
13.70000
13.70000
13.70000
0.10000

13.70000
13.70000
13.70000
13.70000
13.70000
13.70000
0.

100.00000

100.00000

100.00000

100.00000

100.00000

100.00000

0 .

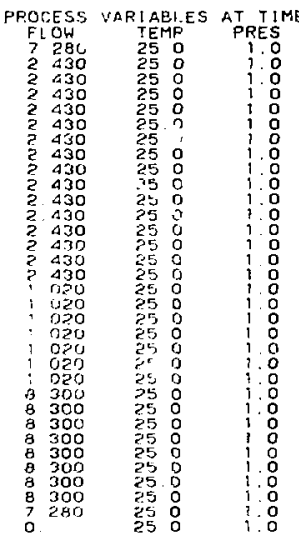

- 00000

- 00000

000006

0 00322

o $i 5890$

7. 21633

7.92722

2. 09803

0 72900

- 22228

o. 05446

o.01110

0.00196

o.
$=2$. OOOOOE+00

COMPGMENTS

9.50000

$0.00107-0.00111-0.00046-0.00119$

$0.00267-0.00048-0.00072 \quad 0.00054$

o.00086 -0.0005i 0.00011 -0.00042

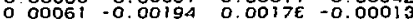

o.00061 0.00090 -0.00010 -0.00009

0.00359 0.00111 0.00142 -0.00057

0.04342 o. 02695 0.01734-0.0011

$0.026950 .0734-0.00112$

$0.43501000440 .11532-0.00120$

$\begin{array}{llll}7.49684 & 3.06977 & 0.33041 & -0.00015 \\ 0.24063 & 0.07834 & 0.01009 & 0.00224\end{array}$

$\begin{array}{llll}0.24063 & 0.07834 & 0.01009 & 0.00224 \\ 0.00548 & 0.00121 & 0.00072 & 0.00057\end{array}$

0. 0007

-0.00130 - -0.00115-0.00125 0.00039

0.0010

o. 00006

$-0.00203$

0.00012 -0 00063 0.00173

$\begin{array}{llllll}-0 & 00032 & 0.00044 & 0.00120 & -0.00023\end{array}$

- 00021 -0.00036 -0.00086 0.00089

0.00089 -0.00216 0.00086 0.00002

$\begin{array}{lrrr}0.00089 & -0.00216 & 0.00086 & 0.00002 \\ 0.00001 & 0.00138 & -0.00052 & 0.00118\end{array}$

0.0006

. 0142

0.00106

$-0.00123$

$-0.00091$

$0.0231\}$

o. 98835

0. 00745

0.00052

$-0.00033$

- 64955

o. 11272

0.77275

0.04683

$1.69446-0.00035$

0.05494

o. 0.3002

0.00457

- 0. 00132

9.50076

\subsection{3}

0.73128

0.25360

o. 25360

$0.01376 \quad 0.01507-0.00122$

0.0026i 0.0032 -0.00234

- 00047 -0.00062 -0 00069

0. $00023-0.00137-0.00116$

4.78004 3. jog86 o. cnoga

13. 70000

13.70000

13. $7 \mathrm{cooo}$

13. 70000

13. 70000

13. 70000

13, 70000

13. 70000

13. 70000

13. 70000

13. 70000

13. 70000

13.70000

o.
100.00000

100.00000

100.00000

100. 00ooo

100 . 00000

100.00000

100.00000

100.00000

100.00000

100.00000

100. 00000

100.00000

ioo ooooo

0 .
100.00000

100.00000

100.00000

100.00000

100. oooor

$100.000 r$.

o.

100.00000

100.00000

100.00000

100.00000

100.00000

100.00000

100.00000

100.00000

100.00000

120.00000

100.00000

100.00000

100.00000

$o$.

Fig. 9. (Comtinued) 


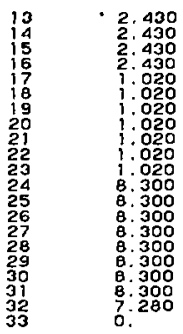

$\begin{array}{rr}25.0 & 1.0 \\ 25.0 & 1.0 \\ 25.0 & 1.0 \\ 25.0 & 1.0 \\ 25.0 & 1.0 \\ 25.0 & 1.0 \\ 25.0 & 1.0 \\ 25.0 & 1.0 \\ 25.0 & 1.0 \\ 25.0 & 1.0 \\ 25.0 & 1.0 \\ 25.0 & 1.0 \\ 25.0 & 1.0 \\ 25.0 & 1.0 \\ 25.0 & 1.0 \\ 25.0 & 1.0 \\ 25.0 & 1.0 \\ 25.0 & 1.0 \\ 25.0 & 25 \\ 25.0 & 1.0 \\ 25.0 & 1.0\end{array}$

$\begin{array}{rr}0.00248 & -0.00026 \\ 0.00101 & 0.00035\end{array}$

0.00031 0.00062 $-0.00035-0.00022-0.00116$ 0.0112 -0.0000 0.00103-0.00036 0.0004 0.000Bo 0.00016 -0.000es 0.00074 - 00050 0.00032 -0.00065 -0.00197 0.00111 -0.00083 -0.00036 $0.00221 \quad 0.00112 \quad 0.00153-0.00117$ $\begin{array}{llll}0.00563 & 0.00301 & -0.00052 & -0.00003 \\ 0.26645 & 0.06431 & 0.00526 & 0.00053\end{array}$

67681

0.7975

0.02283

0.02283

0.01232

0.01111

0.92524

0.15891

0.00095

0.92524

33062

0.00209

0.10106

1. 55268

o. 00043

0.06668

0.40670

o. 00005

0.04627 0.17557 -0.00230

$0.027320 .06770-0.00021$

0.01115 0.02404 -0.00039

0.00391

0. 01115

9. 50028

4.78047

0.00846

0.00033

$\begin{array}{rrr}4.78047 & 3.10133 & 0.00110 \\ 0.00090 & -0.00118 & 0.00029\end{array}$

13. 70000

13. 70000

100 . onวoo

100.00000

o. 00001

13. 70000

100.00600

100.00000

o. 00060

13. 70000

100.00000

100.00000

$0.02 \theta 20$

13. 70000

100.00000

100.00000

1.30073

13.70000

100.00000

100.00000

29. 06150

13.70000

100.00000

100.00000

16. 46328

13. 70000

100.00000

100.00000

3. 82766

13. 70000

100.00000

100.00000

1. 47743

13.70000

100.00000

100.00000

100.00000

o. 76358

13. 70000

100.00000

100.00000

0.40881

13.70000

100.00000

100.00000

o. 19195

13.70000

100.00000

100.00000

0.07620

13. 70000

0 .

o.

100. ooono

o. 10000

o.

Fig. 9. (Continued) 


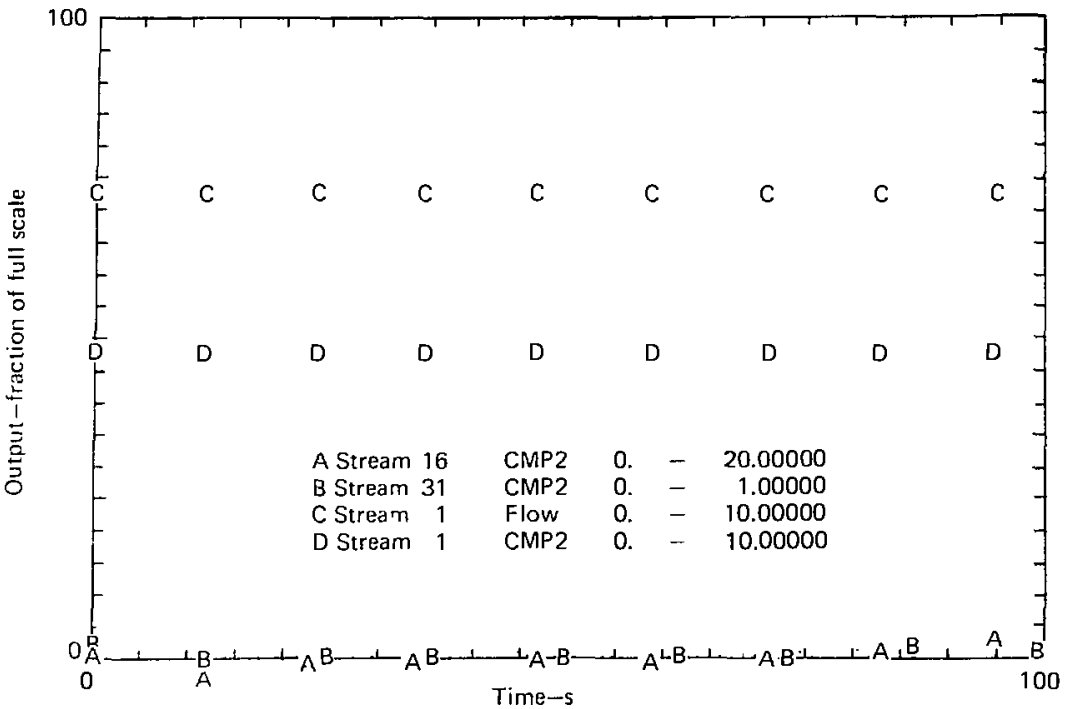

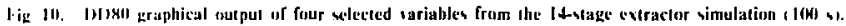

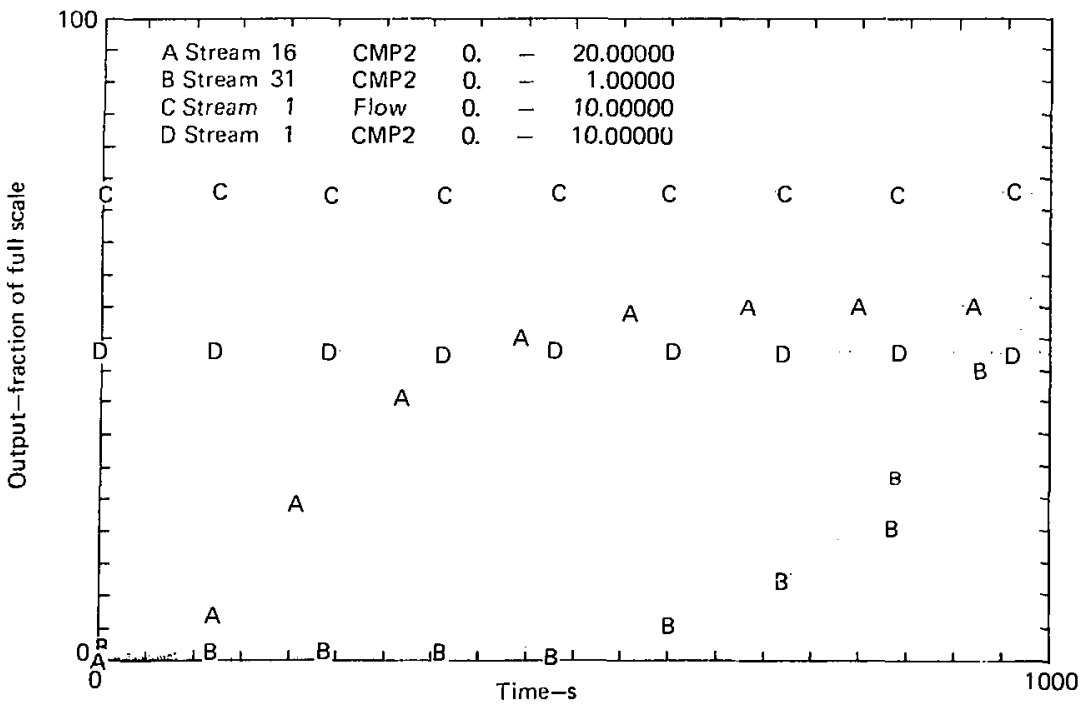

Fip. II. D)[S1) graphical output of four selected tariahles from the 14-stage extractor simalation (1000 s). 


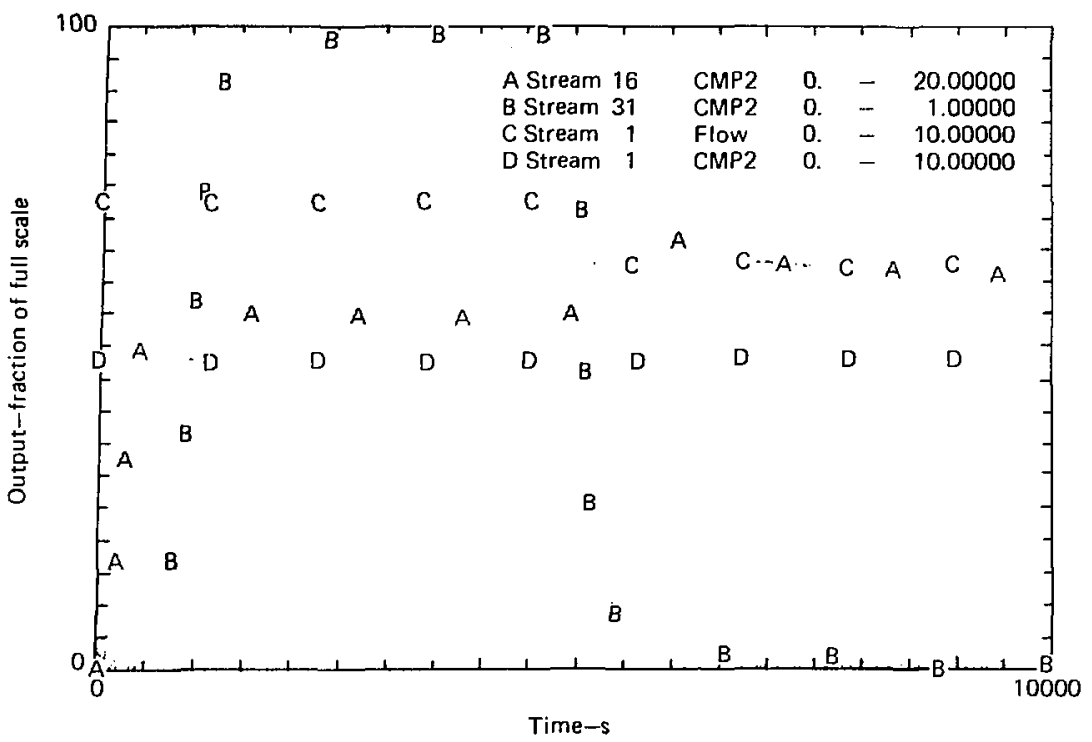

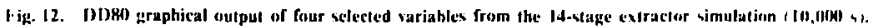

\section{PROGRAM DEFICIENCIES}

The DYNSYL code is now adequate for the dynamic simulation of a short series of units in a chemical plant, particularly one that is composed specifically of the unit modules now available for DYNSYL. The code has several shortcomings, however, that need to be remedied to ensure its most effective use. Some of these are summarized as follows:

- Ir some units are to be coupled and others not, at least two integrator programs are now required, because the GEAR-integrator is not written for such mixed operation.

- The code produces no llowsheet schematic in its output to key into the numerical and graphic output. Such a schematic would aid greatly in providing permanent documentation of the results.

- The graphic and interactive parts of the code are specific to the 1 1.1. computer system and must be rewritten for other computer systems.

- For general use in the chemical industry many mote unit module subprograms must be written. The new modules should be written to be as general as possible with specific applications determined by attached subroutines.

DYNSYL has already proven ukeful in producing sinulated dynamic data for evaluation of online material control methods. Further applications of the code will gradually expand its usefu]ness. 


\section{APPENDIX A \\ UNIT MODULE SUBPROGRAMS}

\section{STGIDI. (Type 1)}

This subprogran as presently written represents one ideal stage for the coextraction of $U+6$ and Pu +4 from other salts. The stage may have two feeds to and two effluents from each phase-organic and aqueous. The volumes of the two phases are constant at predeternined values. The sum of volumetric input flow rattes must, therefore, match the sum of volumetric utput fiow rates. Instantaneous output now raltes are made to match any input llow rate changes. If the aqueous volume is specilied as zero. the module functions as a single-phase, perfutl mixed stage. As structured. the module may very easily be generalized to accommodale chemieal reactions and, therefore, separation of $\mathrm{Pu}{ }^{+3}$ and $U^{+6}$. Also, with a change in ORGPH. the equilibrium program and some statements containing the $U$ and Pu molecular weights, any liquid-liquid extraction may be simulated.

the nomenclature used in the program is as follows:

\begin{tabular}{|c|c|}
\hline V()!.(1) & Organic phase volume (l) \\
\hline VOI.(2) & Aqueous phase volume (I) \\
\hline XTBP & Mass fraction tributyl phosphate in organic solvent \\
\hline KV & Rate constant for approach to equilibrium \\
\hline $\mathrm{KE}: X \mathrm{~T}$ & Rate eonstant for volume change (not used) \\
\hline INSTCiO, INi-1) & Organic input streams $(1.2)$ \\
\hline OUTPRO. OUTSTO & Organic output streans $(3,4)$ \\
\hline INSTCIA. INIDA & Aqueous input streams $(5,6)$ \\
\hline OUTSTA. OUTPRA & Aqueous output streans $(7.8)$ \\
\hline$W(J)$ & Volumetric flow rate for stream $\mathrm{J}$ \\
\hline$T(J)$ & Temperalure for stream $J$ \\
\hline$P(J)$ & Pressure (not a variable) for stream $J$ \\
\hline LIN & Tolal input aqueous flow rate \\
\hline$V 1 N$ & Total input organic llow rate \\
\hline L.OUT & Total output aqueous flow ratt \\
\hline volit & Total output organic flow rate \\
\hline$X \mid N(1)$ & Average input aqueous concentration of component 1 \\
\hline XOUT(I) & Average output aqueous concentration of component 1 \\
\hline$Y I N(1)$ & Average input orginic concentration of component I \\
\hline YOUT(l) & Average output organic concentration of component I \\
\hline TIN & Average input temperature \\
\hline TOUT & Average c utput temperature \\
\hline YIDEAI.(I) & Equilibrium organic concentration of component I \\
\hline DERY(J) & Rate of change of $Y(I)$ \\
\hline DERX(I & Rate of chunge of $X(I)$ \\
\hline $\operatorname{DISCO}(\mathrm{I})$ & Ratio of YIDEAL (I) to $X(I)$ \\
\hline
\end{tabular}

The equipment parameters, EP(IM,J), are as follows:

$\operatorname{EP}(\mathrm{IM} .1)$

$\mathrm{EP}(\mathrm{IM}, 2)$

$\mathrm{EP}(\mathrm{IM}, 3)$

EP(livi,4)

$E P(I M . j)$

EP(IM,6)
Plutonium holdup, grams

Organic phase volume (I)

Aqueous phase volume (I)

Rate constant for volume change

Rate constant for approach to equilibrium

Weight fraction tributyl phosphate in organic phase 


\section{CONTIR (Type 2)}

This module is a simple ilgebraic proportional-plus integral control element. Its input streum $X$ is information from a stream (temperature, concentration. Now rate). The output is a signal to a control device, such as a valve (VAI.V). If the deviation from a set point is $\mathrm{F}=(\mathrm{X}$-set point)/range, the output signal $\mathrm{Y}$ is given by $\mathrm{dy} / \mathrm{dt}=\mathrm{K}_{p}\left(\mathrm{dl} / \mathrm{dt}+\mathrm{K}_{\mathrm{l}} \mathrm{I}\right)$, where $\mathrm{K}_{p}$ and $\mathrm{K}_{\mathrm{I}}$ are proportional gain and integral conslant, respeclively. The module simulates this equation without using an outside inlegrator.

The nomenclature used in the module is as follows:

$\begin{array}{ll}\text { PI } & \text { Proportional gain } \\ \text { P2 } & \text { Integral constant } \\ \text { IN } & \text { Inpul slream } \\ \text { OITT } & \text { Output signal } \\ \text { K } & \text { Variable index } \\ \text { OI.I) } & \text { Old value of variable } \\ \text { I:RR } & \text { Deviation from set point } \\ \text { OI.IXI:R } & \text { Old deviation }\end{array}$

The equipment parameters to be specified are:

$1: P(13,1)$

$\operatorname{t.P(IM.2)}$

[FP(IM, 3)

I:P(IM.4)

I.P(IM.5)
Controlled variable index

Range of controlled variable

Set point of controlted variable

Proportional gain

Inlegral constant

\section{PIPl: (Type 3)}

Simulation of flow through a pipe is, for purposes of dynamic plant modeling. cescintially time-delay w ith dispersion. If the pipe is relatively long, the dispersion may be ignored. so this module is a pure delay $\mathrm{It}$ is a modilication of $D 1 . A Y$ in the original DYNSYS program. The delay ime mat be fixed or based on pipe length and lluid velocily. The delay is atcomplished by storing values of the stream variables for the correte number of time inerements, and then using the values previously stored as input (exit from the pir. $\because$ ). It is possible to delay only a selected part of a stream. making a crude sinulation of dispersion possible. Vo intepration is involved in this module.

The nomenclature is as follows:

$\begin{array}{ll}S X(I, J, K) & \text { Matrix of delayed stream values } \\ I & \text { Index of past times in } S X \\ J & \text { Matintilined as I in } S X \\ K & \text { Index of stream variables } \\ \text { IN } & \text { Input stream (output from upstream unit) } \\ \text { OUT } & \text { Outpul stream (input to downstrean unit) } \\ \text { TLACi } & \text { Value of delay time } \\ \text { BYP } & \text { Fraclion of stream not delayed } \\ \text { NY } & \text { Number of storage spaces in de'sy vector } \\ \text { HH } & \text { Time increment }\end{array}$

The equipment parameters that musi be specified are: 


\section{EXTRTR (Type 5)*}

This is a module for multistage extraction and chemical reaction simulation. It is bused on a program written by L. 1: Burkhart of lowa State University, and is capable of simulating plutonium-uranium scparation or coextraction with variable phase volumes. The module does not account for any backmixing or dispersion effects. The number of equilibrium slages may be specified up to 20: the feed stage may also be specified. The stage volumes are uniform and are determined from the specified column volume. It is also pussible to specily the time constant for changes in the phase volume ratio. Integration occurs within this module. so the integrator portions of DYNSYL are not used.

$A$ sehematic of the extraction column modeled is shown in Fig. $\mathrm{A}-1$ with names of some of the variables shown. The nomenclature used in the module is as follows:

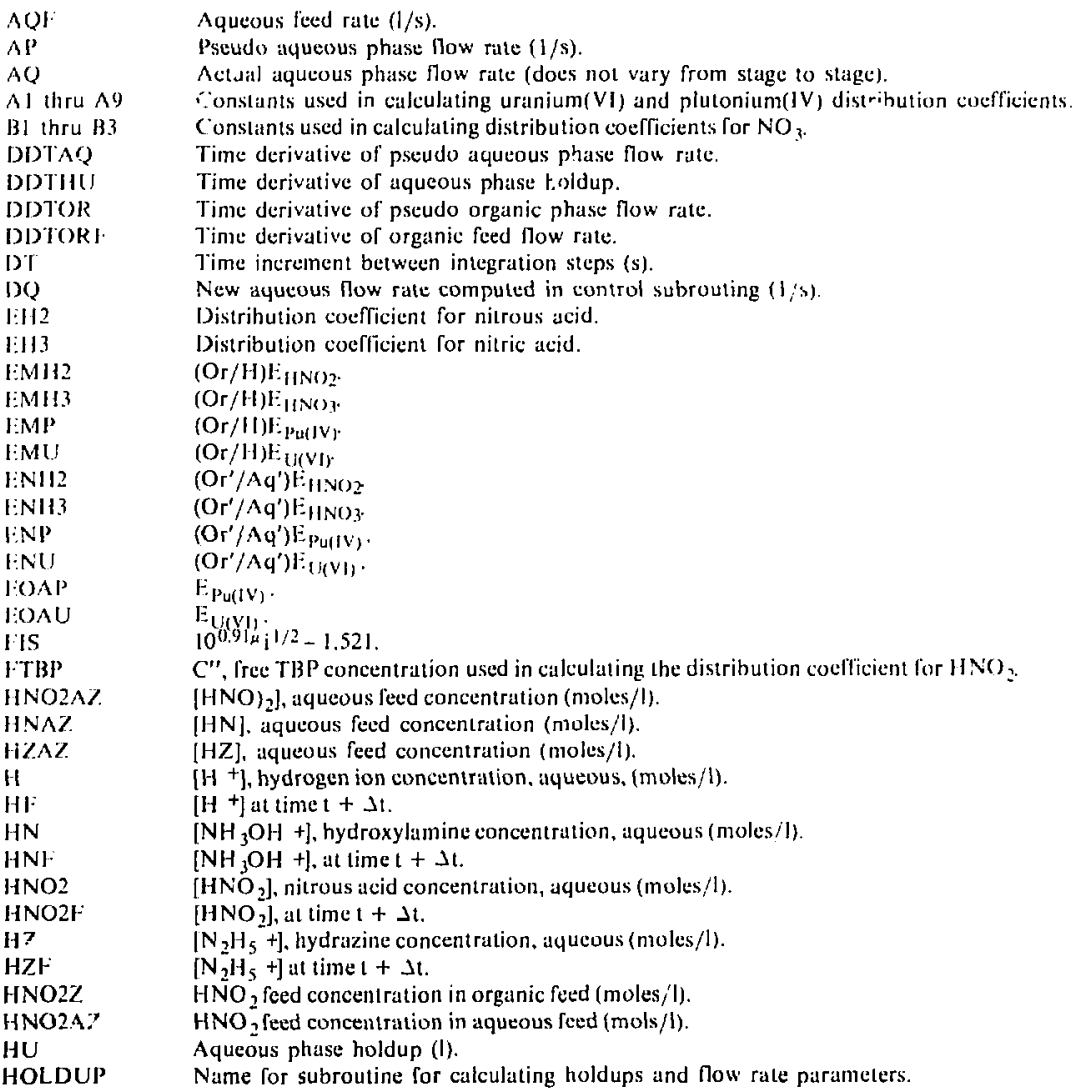

-There is no lype 4. 


\begin{tabular}{|c|c|}
\hline $\mathrm{KH}$ & $K_{1}$, ionic strength function for $[H+]$. \\
\hline KP & $k_{\text {Purli: }}$ \\
\hline $\mathrm{Kl}$ & $k_{1} / 1 r$ \\
\hline $\mathrm{Kl}$ & Rate constant for reaction 1 . \\
\hline K2 & Constant used in TEMA in material balance calculations. \\
\hline K? & Rate constant for reation 3 . \\
\hline K- & Ralte tonstant for reaction 2 . \\
\hline K.5 & Rate constant for reaction 4 \\
\hline I. & Number of equilibrium stages in the column. \\
\hline$\$$ & $\begin{array}{l}\text { Si mbol for fictitious stige }\left(1_{-}+1\right) \text {. used to specify inputs and outputs at the } \\
\text { buttom stige (L.). }\end{array}$ \\
\hline 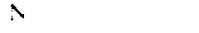 & Number of iterations or time steps specified. \\
\hline NI. & leed slage number. \\
\hline $\mathrm{NO}$ & $V()_{3}$ conkentration in aqueous phase (moles /J). \\
\hline $\mathrm{N}(0.30$ & Vi) concentration in organic phase (moles $/$ l). \\
\hline $\mathrm{N}(031$ & $|>0$,$| at time 1+\Delta 1$. aqueous. \\
\hline $\mathrm{S} 0,3 \%$ & $\mathrm{Sew} \mathrm{VO}_{3}$ concentration in organic fered (noles;l). \\
\hline$\times 0,37$ & 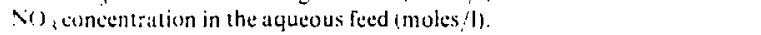 \\
\hline$\therefore 7$ & (NI -1). stage hefore feed stage. \\
\hline$x 8$ & $(N I+1)$, stage after feed stage. \\
\hline 0 & Pseude organic phase flow rate. \\
\hline C & () Fitule phase thow rale (does not vary from stage to stage) $(1 / \mathrm{s})$ \\
\hline ORI & Organic feed flow rate immediately preceding a flow rat. upset $(1, s)$. \\
\hline (נR। & Organic phase flok rate in scrub section (1/s). \\
\hline$\left(3 R_{2}\right.$ & 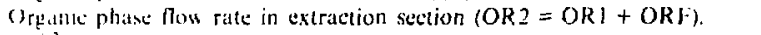 \\
\hline 13.1 & P'u ${ }^{3}$ concentration in aqueous phase (moles $/ \mathrm{l}$ ). \\
\hline 9331 & fut al time $t+\lambda t$ aqueous. \\
\hline$p+1$ & $\mathrm{Pu}^{+}+$concentration in aqueous phase (moles $/ \mathrm{l}$. \\
\hline$P A N I$ & $P_{u}+4$ at time $t+\Delta t$ \\
\hline$p^{2}+()$ & Pu ${ }^{+4}$ concentration in organic phase (moles $/$ ). \\
\hline $13+0)$ & $\mathrm{Pu}+4$ concentration in organic feed (moles/l). \\
\hline$P+10 \mathrm{M}$ & $\begin{array}{l}\mathrm{Pu}^{+4} \text { concentration in urganic feed immediately following a Pu feed concen- } \\
\text { fration upset (moles/1). }\end{array}$ \\
\hline P'BM & Revidual from overall plutonium material balance over the column. \\
\hline $\mathrm{RN}$ & $-1 . A_{4} \cdot\{\}$ \\
\hline RXVI Iiru RXVis & $\begin{array}{l}\text { Incremental thange in muterial gained or lost to reaction for each time step } \\
\text { as fetermined by the kinetic rate equations. }\end{array}$ \\
\hline 1.30 & $i_{4}$. interstaze time constant for pseudo aqueous flow rate $\left(s^{-1}\right)$ \\
\hline TOR & Tor. interstage time constant for pseudo organic flow rate $(s-1)$ \\
\hline$(0)$ & $\left[\left(V^{\prime} l 1\right)\right]_{(\text {r. }}$ organic feed concentration (moles $\left./ 1\right)$ \\
\hline 13 & {$[1(1]]_{\mathrm{Aq}}$ itqueous feed concentration (moles $/ I$ ). } \\
\hline$l^{\prime} x$ & {$[\mid(1 \mathrm{l})]_{\mathrm{Ay}}$, aqueous feed concentration (noles/l). } \\
\hline l'sl & {$[l(V)]_{\mathrm{Ay}^{4}}$ at timet + $\mathrm{ti}$} \\
\hline ( 0 & {$\left[\mathrm{~L}(\mathrm{~V} /)_{\mathrm{O}_{\Gamma}}, \mathrm{U}+4 \mathrm{concentration}\right.$ in the organic phase (moles/1). } \\
\hline 103 & $\begin{array}{l}\text { Lranium concemtration in feed at steady-state immedjalely preceding an up- } \\
\text { set in uranium feed concentration (moles/l). }\end{array}$ \\
\hline $\mathrm{VOH}$. & Volume of a single theoretical stage (I). \\
\hline
\end{tabular}

The equipment parameters required by this module are:

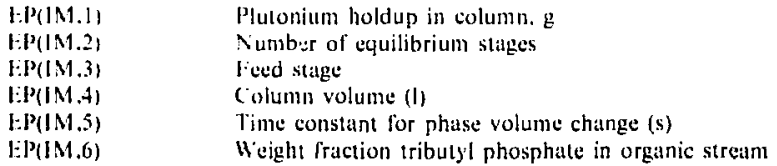




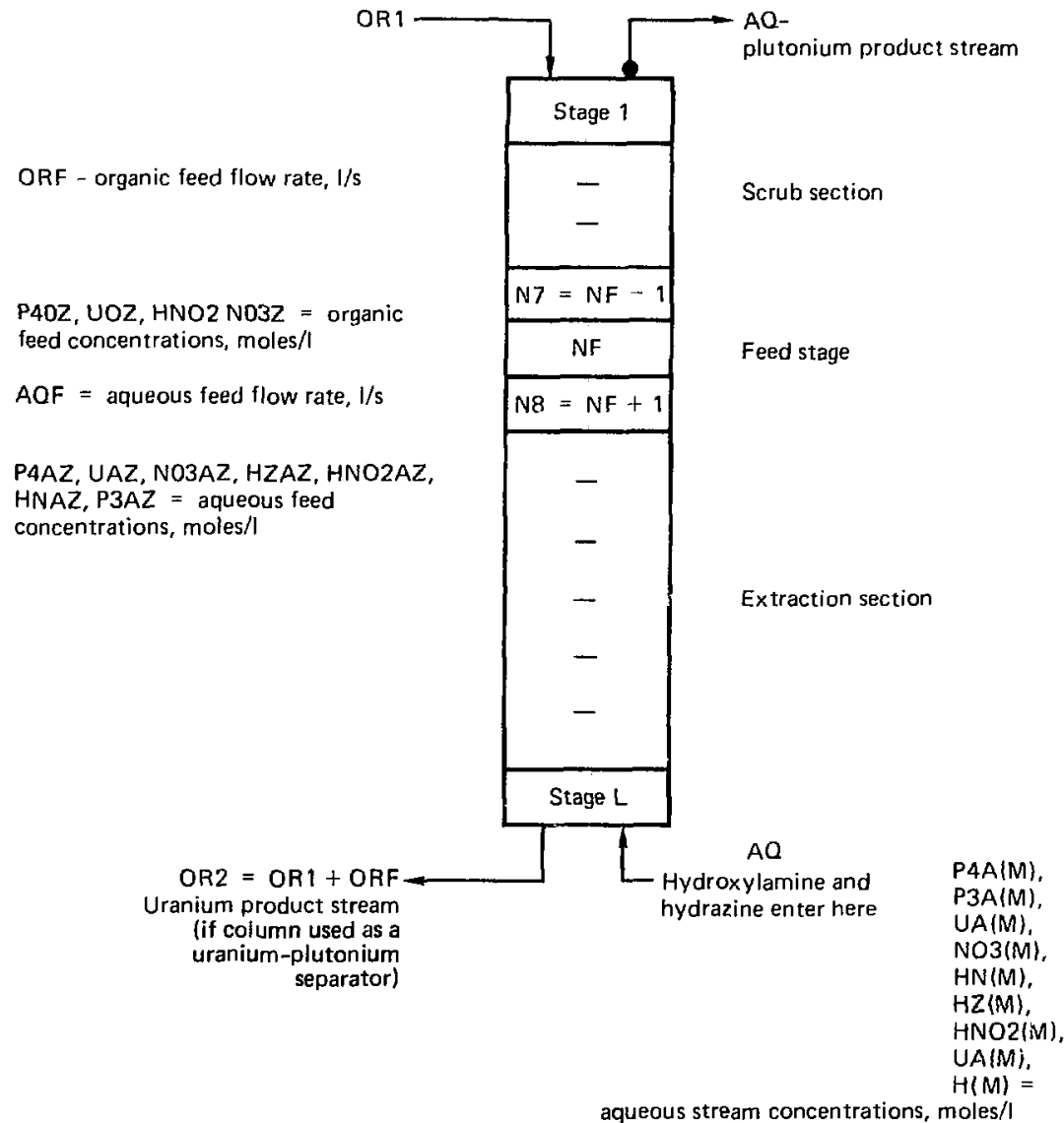

Hife. A-1. Fixtruction column sbowing location of streams and principal variables used in computer program. 


\section{PRECIP (Type 6)}

This module simulates the dynamic behavior of a precipitator-reactor for the precipitation of piutonium as oxalate. i major component of the simulation is involved in the computation of nucleation and growth rates of the precipitated $\mathrm{Pu}\left(\mathrm{C}_{2} \mathrm{O}_{4}\right)_{2}$ particles. Reference 15 details the derivition of the equations used to model the particle nucleation and growth process in the reactor. To use the module it is only necessary to specify the reactor volume as the nucleation and growth rate constants are specified in the program. Two streams nust enter the reaclor, one containing the plutonium solution, the other the oxalate solution. Only one stream exits the reactor-a mixture of filtrate and precipitate.

The nomenclature used in the moule is as follows:

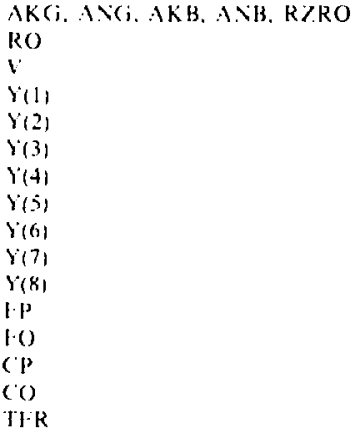

$A K C ;, A N(i, A K B, A N B, R Z R O$

Constants in nucleation and growth equations

$\mathrm{Pu}\left(\mathrm{C}_{2} \mathrm{O}_{4}\right)_{2}$ particle density (g/cc)

Reactor volume (l)

Concentration of plutonium in reactor solution (filtrate), (g/l)

Filuate flow rate $(\mathrm{l} / \mathrm{h})$

Concentration of $\mathrm{NO}_{3}^{-}$in ruactor solution (moles/l)

Concentration of $\mathrm{C}_{2} \mathrm{O}_{4}$ in reactor (moles/l)

Zero moment of distribution

first moment of distribution

Second moment of distribution

Third moment of distribution

Plutonium-solution feed rate $(i / h)$

Oxalate-solution feed rate $(\mathrm{l} / \mathrm{h})$

Concentration of plutonium in feed strum $(\mathrm{g} / \mathrm{l})$

Concentration of oxalute in oxalate-feed stream (moles/l)

Tolal feed rate $(1 /$ h)

The equipment parameters are:

$\begin{array}{ll}\text { I:P(IM.1) } & \text { Plutonium holdup (g) } \\ \text { I:P(IM.2) } & \text { Reactor volume (l) }\end{array}$

The output ruriables are as follows:

S(2.NOUT.6)

S(2,NOUT.7)

S(2,NOUT.8)

S(2.NOUT.9)

S(2.NOUT.10-12)
Rate of change of foed flow rates

Rute of change of plutonium concentration in filtrate

Rate of change of $\mathrm{NO}_{3}^{-}$-concentration in filtrate

Rute of change of $\mathrm{C}_{2} \mathrm{O}_{4}^{-}$-concentration in filtrate

Rates of change of particle size distribution moments

\section{EVAPTR (Type 7)}

This module is the simplest possible version of an evaporator module that may be used as a plutonium-solution concentrator simulation, It is an adaptation of the perfectly-mixed evaporator writsen by Rudolf Rozsa at LLL. The solution properties were all internally generated and were fixed in the earlier program. This program was generalized to use variable properties (density, heat of vaporization, heat capacity) computed by the property subroutines in DYNSYL. We integrated analytically in this module because the balance equations were linear. 
The nomenclature used is as follows:

$\begin{array}{ll}\text { IN } & \text { Input stream } \\ \text { OUT } & \text { Output stream } \\ \text { ViSP } & \text { Vapor stream } \\ \text { STEAM } & \text { Steam stream to heater } \\ \text { MFIN } & \text { Feed rate (moles } / \mathrm{h} \text { ) } \\ \text { XIPU } & \text { Plutonium mole fraction in leed } \\ \text { MSTM } & \text { Steam rate (moles } / \mathrm{h} \text { ) } \\ \text { PVPS } & \text { Vapor presure } \\ \text { PRESS } & \text { Presure in vapor stream } \\ \text { TIEMP } & \text { Temperature of vapor stream } \\ \text { DAVGOUT } & \text { Densily of product liquid } \\ \text { XOPU } & \text { Plutonium mole fraction in product } \\ \text { V } & \text { Evaporator volume (liquid phase) }\end{array}$

The only equipment parameters are:

$\begin{array}{ll}\text { EP(IM,1) } & \text { Plutonium holdup (g) } \\ \text { EP(IM,2) } & \text { Volume (i) } \\ \text { CPi } & \text { Ieat capacity of stream -i } \\ \text { RHOi } & \text { Density of stream -i }\end{array}$

\section{GNTRNS (Type 8)}

This is a simulation of a unit capabie of heat transfor, two-phase equilibrium (liquid-liquid or vaporliquid), and chemical reaction. The module is not yet perfected and more changes will undoubtedly be made. When perfected, the module will be able to simulate the dynamic behavior of ideal extraction and distillation (or flash) stages, mixed reactors, evaporators, heat exchangers, or condensers.

The nomenclature used in GNTRNS is the same as in STGIDL, with some additional terms. These ure as follows:

$\begin{array}{ll}\text { HCOJF } & \text { External heal transfer coeflicient } \\ \text { HAREA } & \text { External heal transfer area } \\ \text { NTYPE } & \text { Zero for extraction; onc for flash } \\ \text { HT etc. } & \text { Heat capacity }\end{array}$

The equipment parameters 10 be specified are as follows:

Plutonium holdup (g)

Total volume (l)

Aqueous or liquid phase volume (l)

Rate constant for volume change

Rate constant for approach to equilibrium

Volume fraction tributyl phosphate

External heat transfer coefficient $\left[\mathrm{cal} /\left(\mathrm{cm}^{2} \cdot \mathrm{s} \cdot{ }^{\circ} \mathrm{C}\right)\right]$

External heat transfer area ( $\left(\mathrm{cm}^{3}\right)$

$E P(I M, 9)$

Stage type: 0 for extraction, I for nish 


\section{VALV (Type 9)}

As written, this module simulates the simplest possible contro' valve or electrical current controller. The output rate is simply proportional to the controller signal or proportional to the maximum controller sigral ( 1.0 in th sase) minus the current signal. Generalization to include other actions would be very simple.

The nomenclature is:

$1 \mathrm{X}$

OL:T

Input signal from controller (CONTLR)

(ontrolled stream flow rate (or current if stectrical)

The unit parameters are:

1.P(IM.1)

Proportionally constant for valve action.

EP(IMI.2)

Posilive causes proportional action: negative causes reverse proportiona' action. 


\section{APPENDIX B \\ PROGRAM LISTING WITH \\ UNIT MODULE SUBPROGRAMS}

The DYXSYl code hat many comments that help explain each section whe uxer. We hate ch. deivored to make the unit module subprogram even more selfexplanatury. The code is listed with the primary sectums in the following order: main and input-output. physical properties. integritor, and unat modules. the arder of the specific subroutines and their purpose is as follows:

\begin{tabular}{|c|c|}
\hline MAIN & (alling progratm \\
\hline DYNI & Numerical input and initializaton \\
\hline (it:T & Alphameric input \\
\hline DYN2 & Linil modulc calling program \\
\hline OHTH:TS & Vumerical output of state variables \\
\hline SAVR:P & Computation of plot variables \\
\hline WRITIP & Oulput of measurement vector \\
\hline I.PIOO'I & I.meplot output \\
\hline CAIPI & DD80 plot routine \\
\hline PROPS & Read constants for physical properlacs \\
\hline MOWJ & Average molecular weight \\
\hline C M & Average liquad heat capacity \\
\hline$(P V A)$ & Average : apor heat capatcily \\
\hline H.NII & Average liquid enthalpy \\
\hline INTV & Average vapor enthalpy \\
\hline I.AMis & lleat ol vaporigation \\
\hline DI:NI. & Average liquid molal densily \\
\hline DINV & Average vapor molal density \\
\hline VAPR & Vapor pressure \\
\hline WII.S & Aclivity coefficients \\
\hline |3【」ß!. & Bubble point \\
\hline KVAI. & Vapor-liquid equilibrium ratio \\
\hline [H:MPl. & I.iquid heat balance \\
\hline TH:MPV & Vapor heat halance \\
\hline DRIVI: & Main program of integrator \\
\hline NOISE: & Process and measurement noise \\
\hline SIII-F: & Integration control program \\
\hline PSIT & Computation with Jacohian \\
\hline INTFRP & Intcrpolation of state variables \\
\hline COSI:T & Integration coefficients \\
\hline DI:C & Matrix triangularization \\
\hline SOl. & Solution matrix equations \\
\hline STCIIDI. & Cieneral ideal stage \\
\hline EXTRTR & Multistage extraction for uranium and plutonium \\
\hline PRIECIP & Precipitator for plutonium oxalate \\
\hline EVAPTR & Evaporative concentrator for $\mathrm{Pu}\left(\mathrm{NO}_{3}\right)_{4}$ \\
\hline CONTLR & Controller \\
\hline PIPE & Flow delay anu dispersion \\
\hline PUMP & Flow driver and head source \\
\hline
\end{tabular}




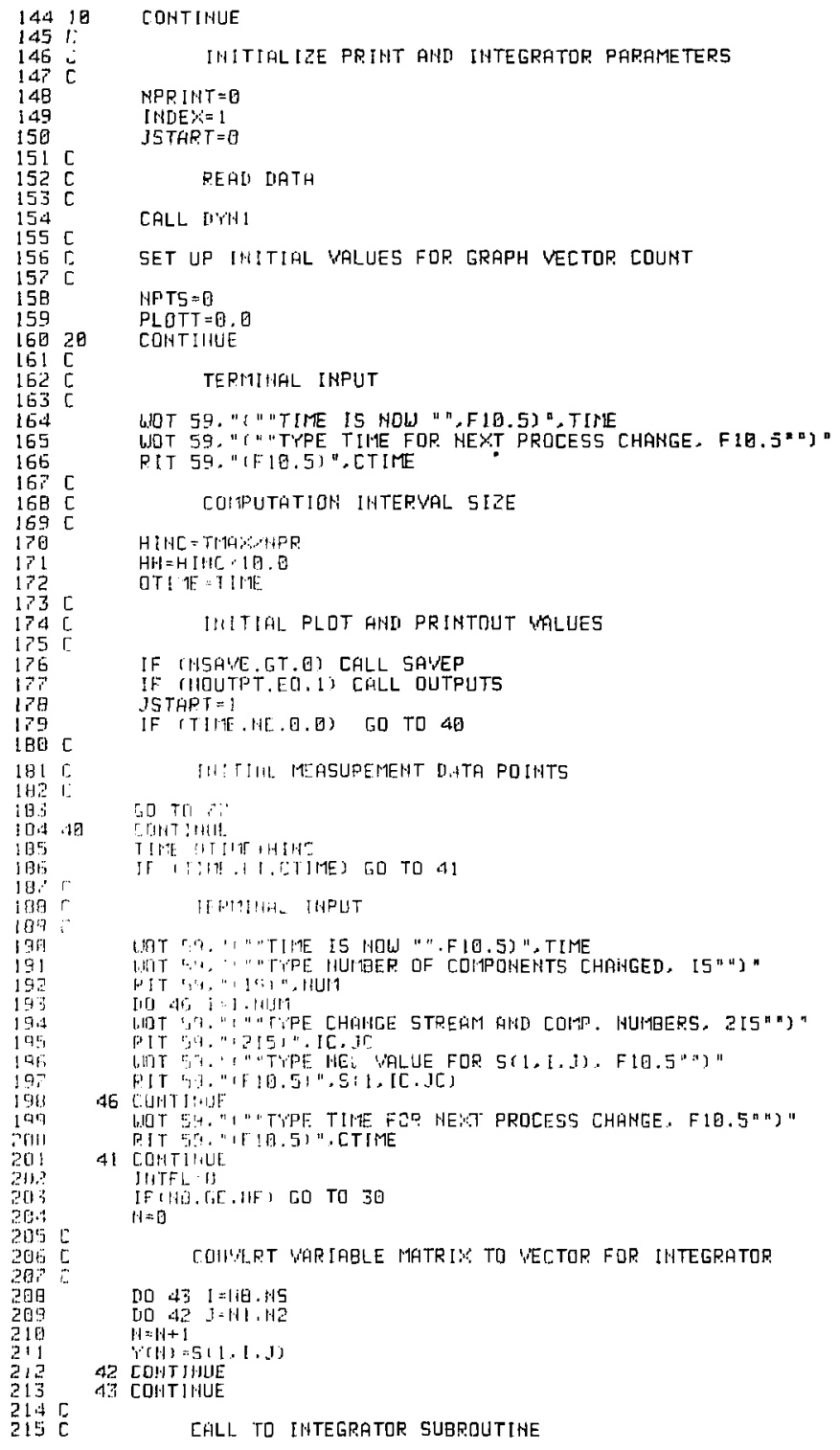




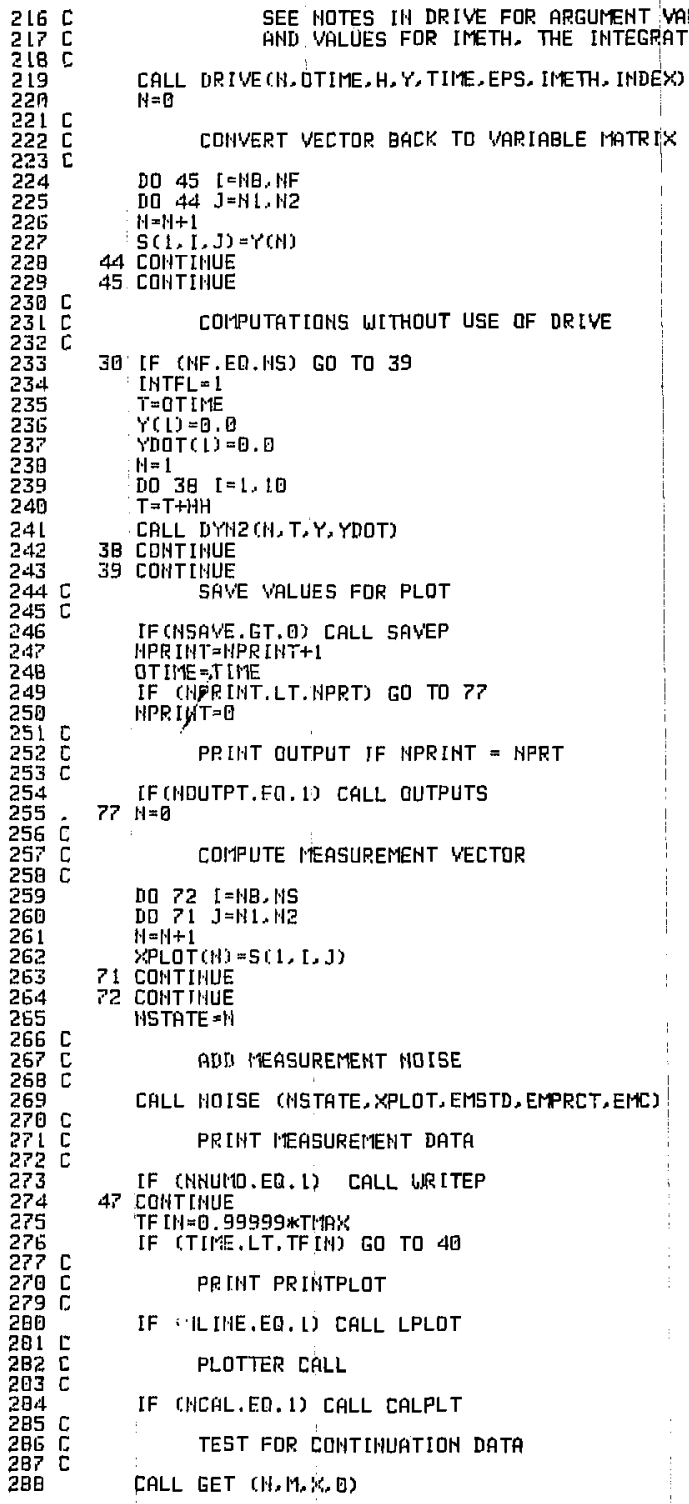




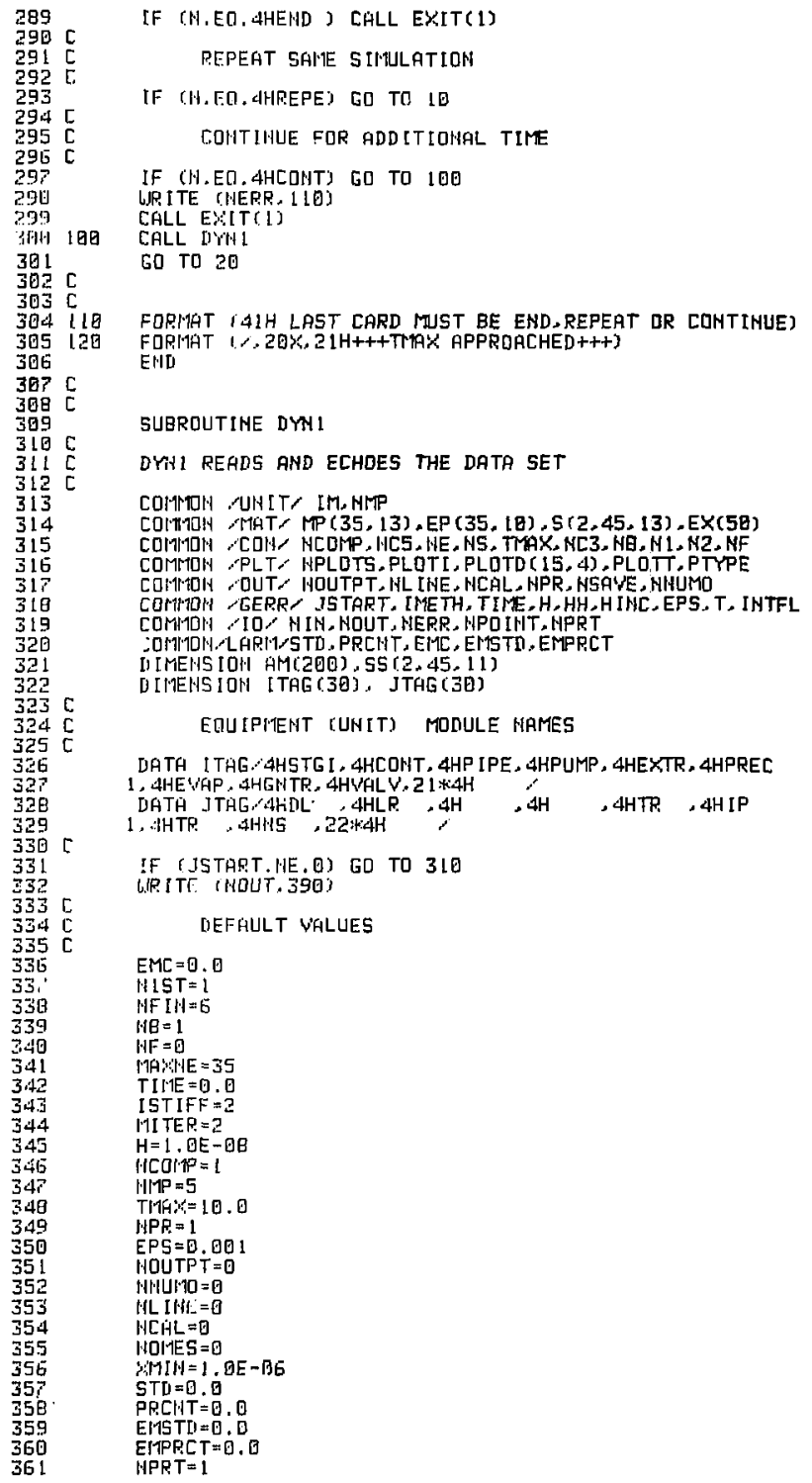




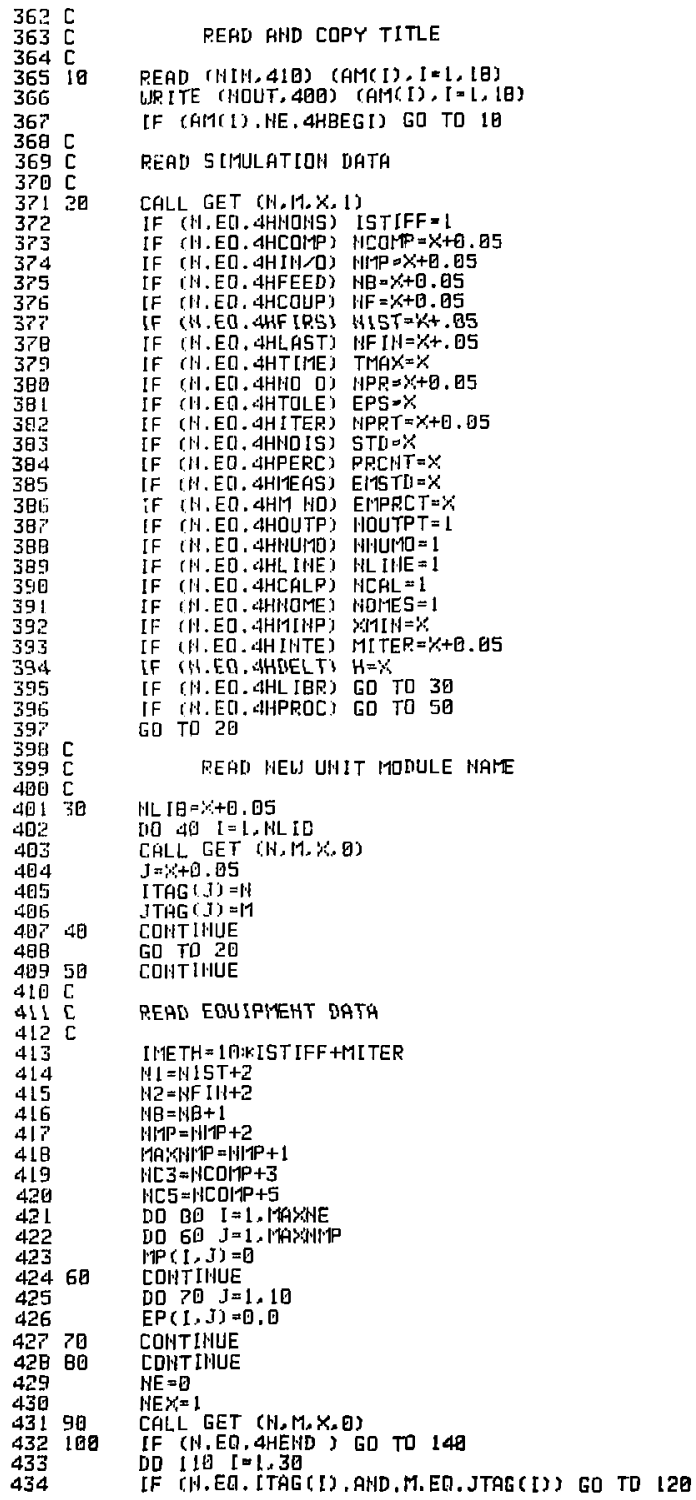




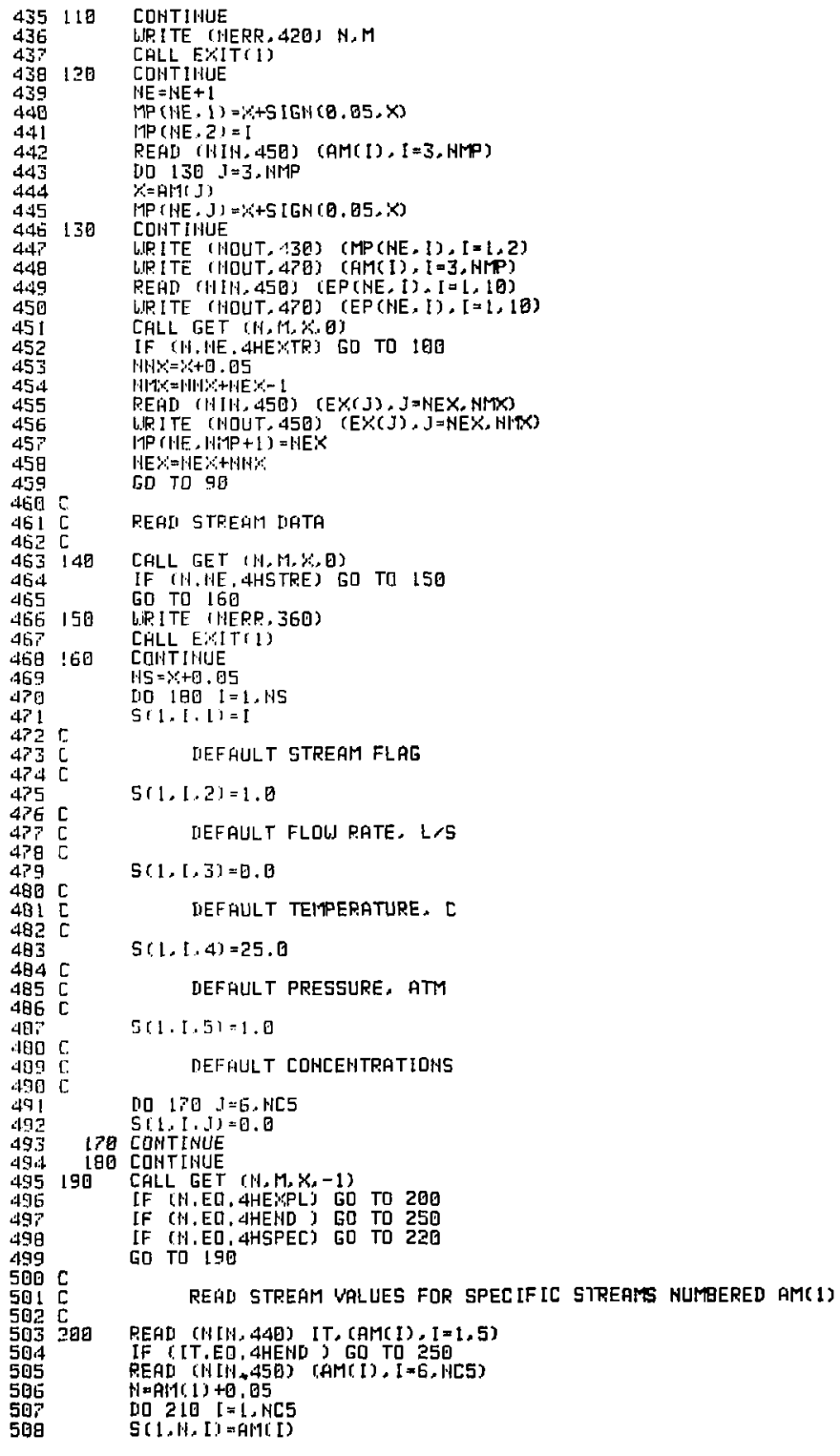




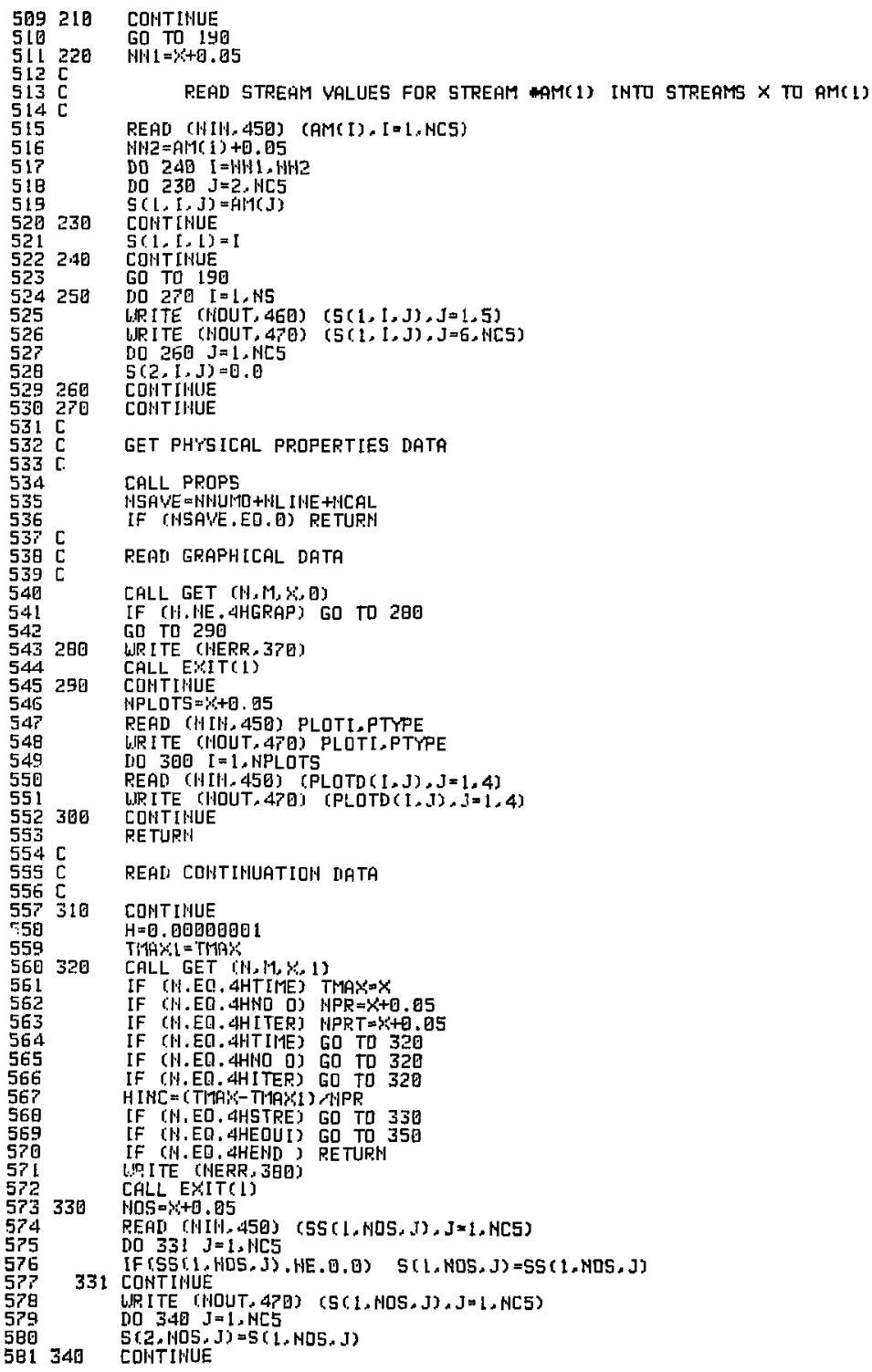




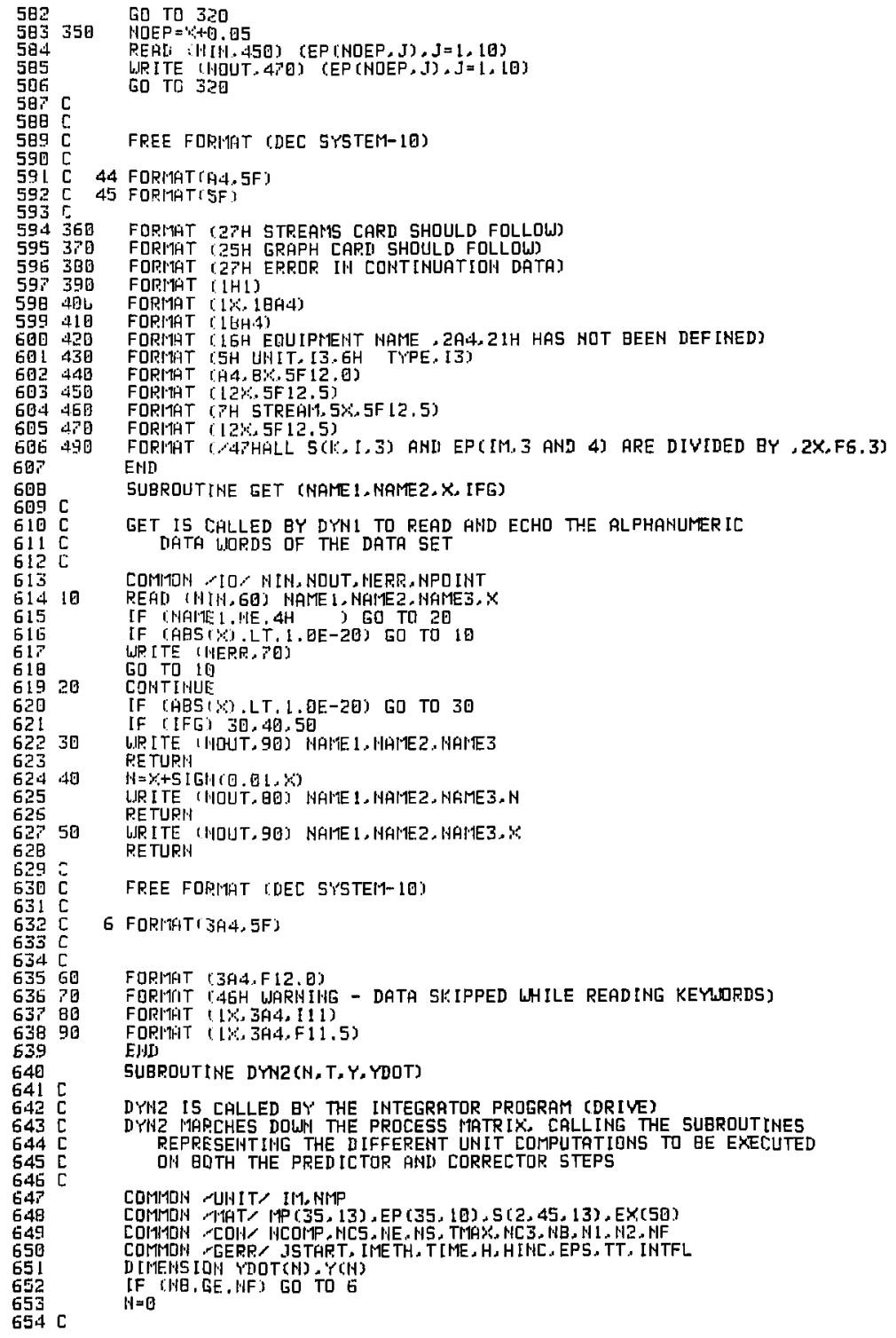




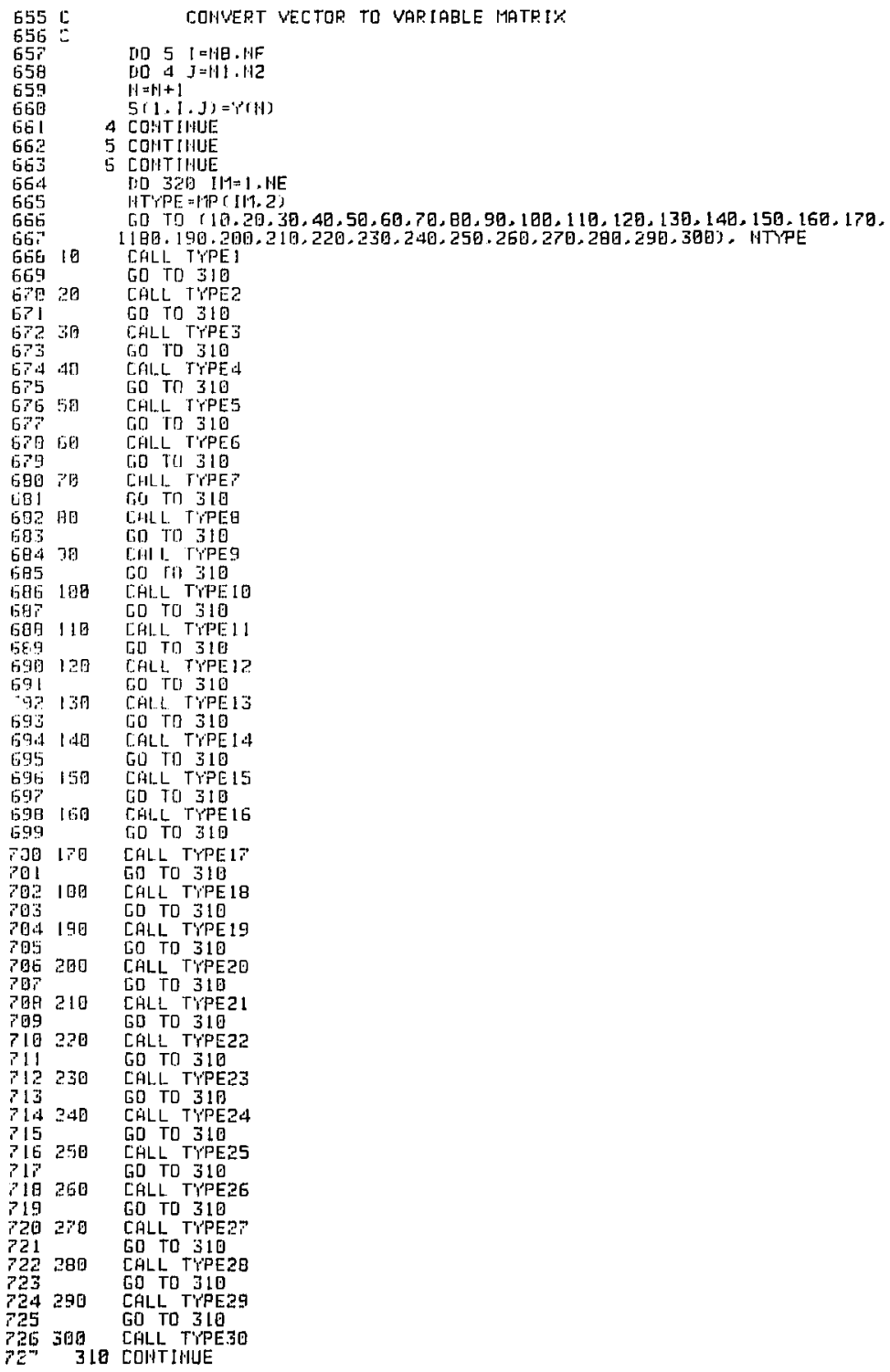




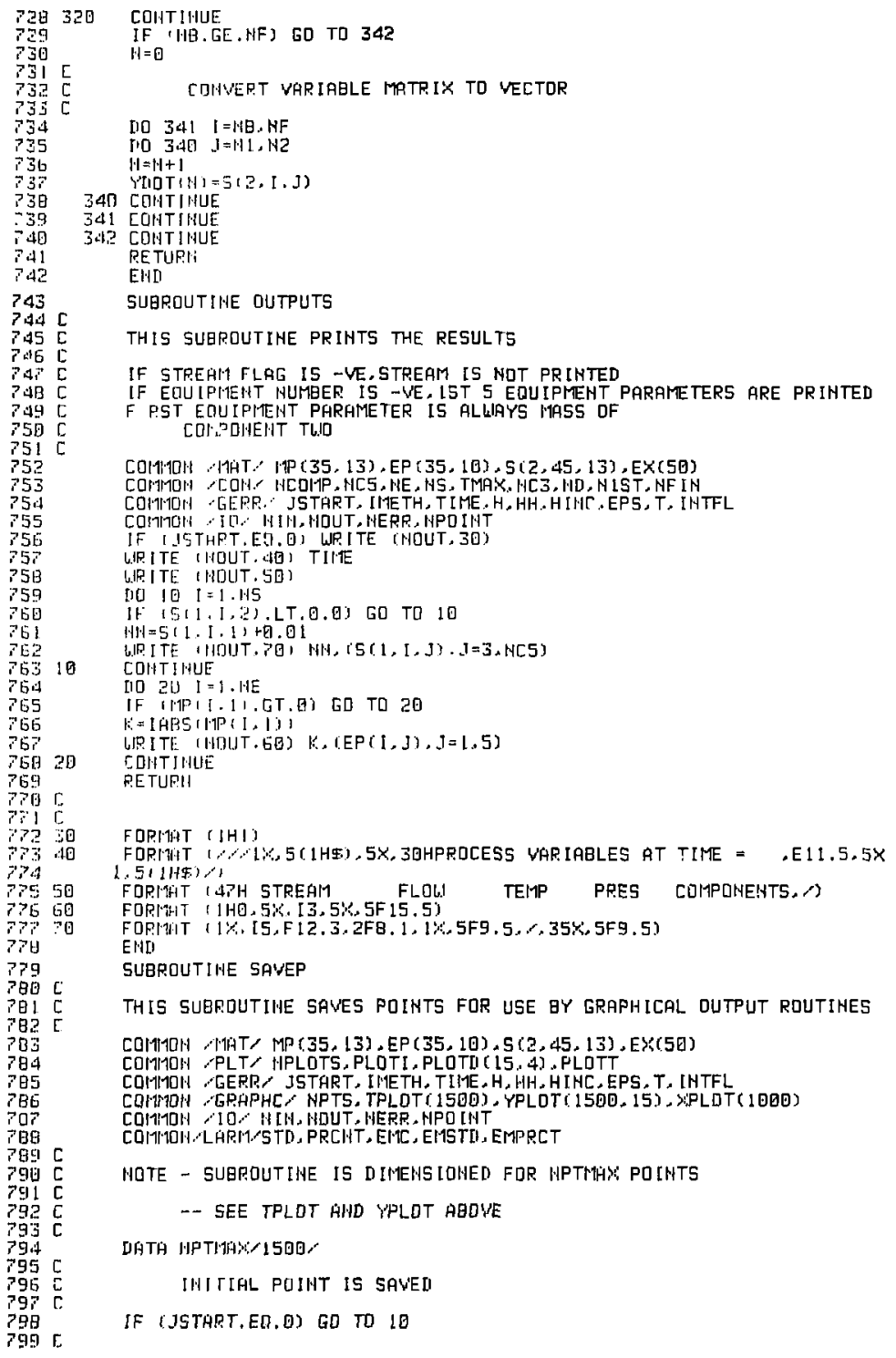




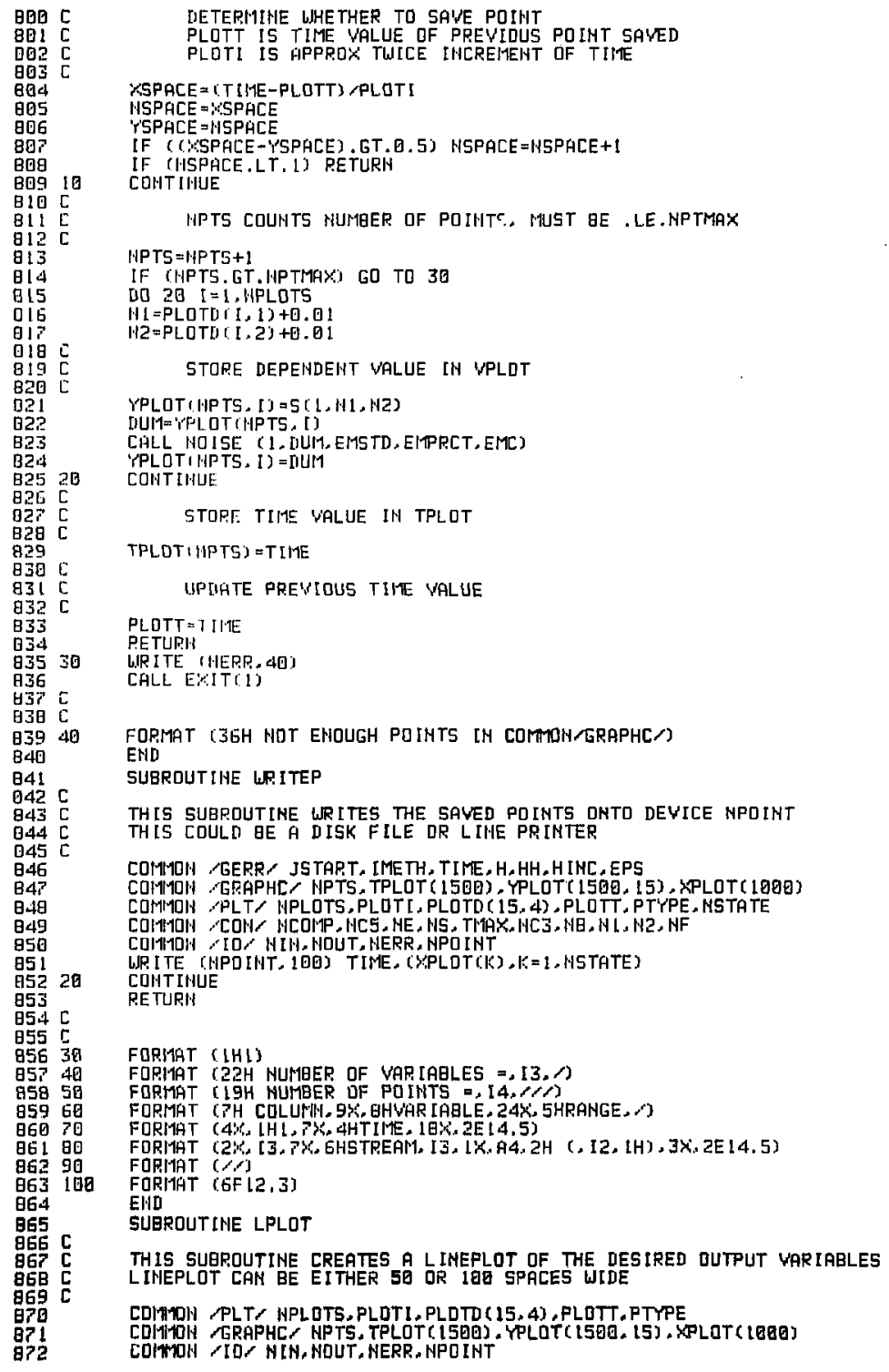




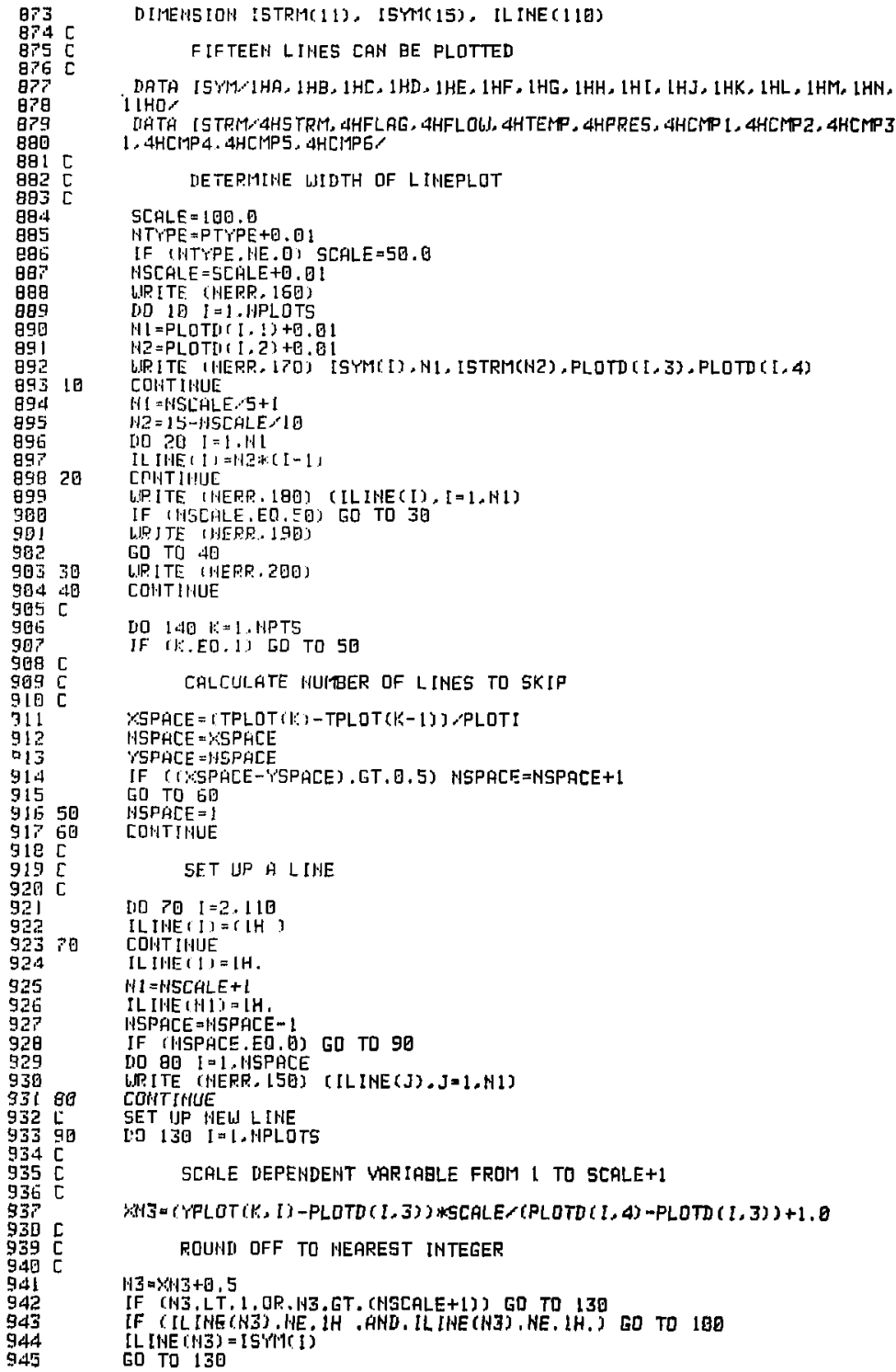




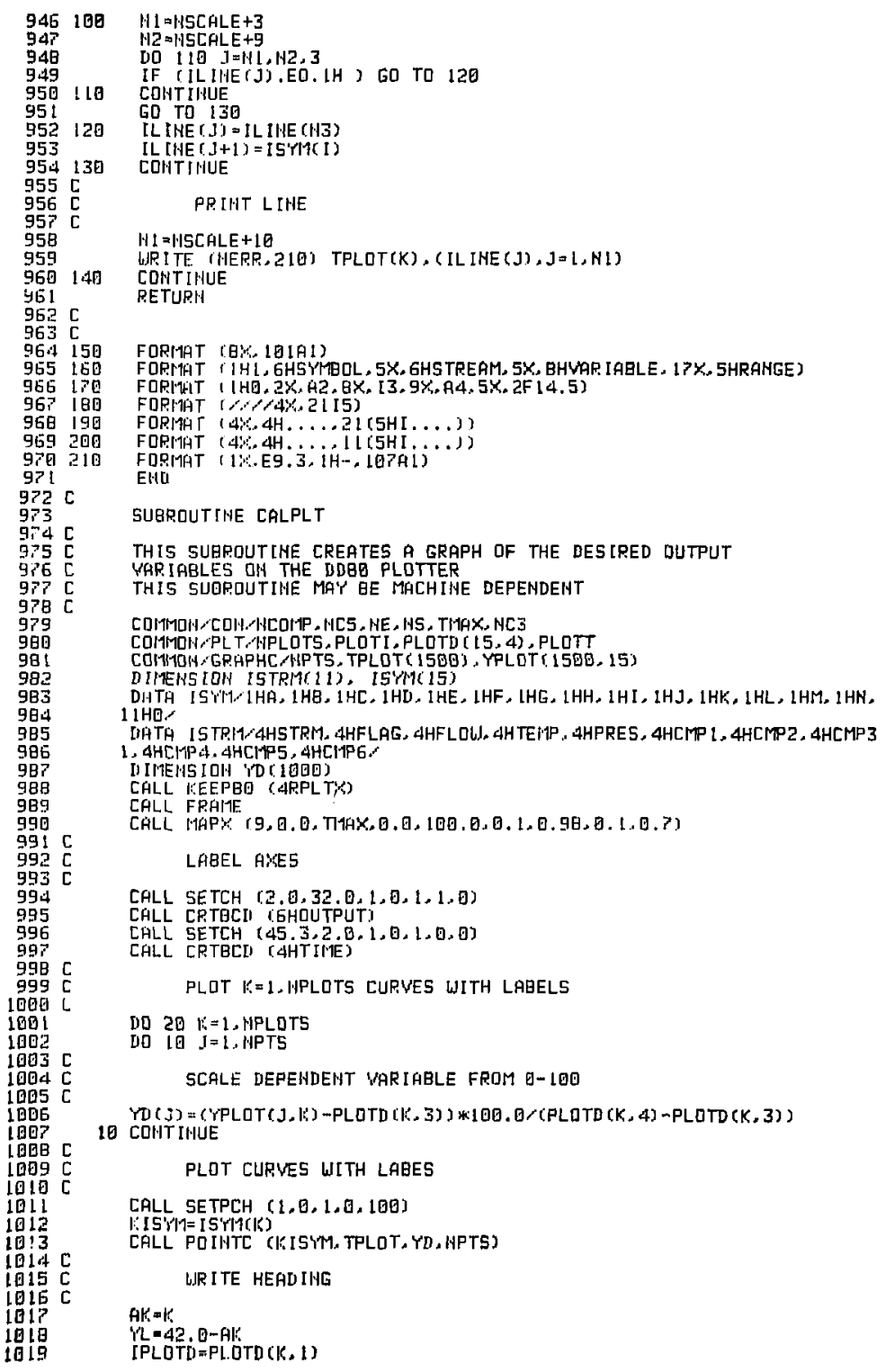


MPLQT =PLOTI (K.2)

CALL SETCH (25,0,YL, L, O, L, 0, G)

LFP ITE (100, 40) ([SYM(K), [PLOTD, ISTRMCMPLT), PLOTD $(K, 3)$, IPLOTD (I:. - 4)

40 FORPHT (H1, QH STREAI , [2,3K, H4,3X,F 12,5.3H -,F12.5)

20 CONTIHUE

CFLL SETCH $(6.0 .30,0,1,0,2,0.0)$

CALL CP.TBC[ (EHLEGEHD)

R.ETURI

EHD

SUBROUTIHL PROPS

THIS SUBROUTINE READS THE CONSTHAITS FOR COMMTH PHYSICAL PPOPERTY CORRELATIOHS PRIPERTIES OF UHTER OR AIP MAY BE SPECIFIED

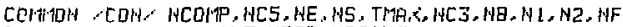

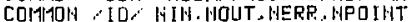

COPTTO IPTAB, TREF. P.

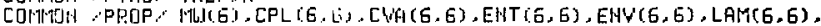

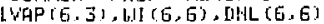

COI1MTH IIPPOP HCP, ME $\therefore$ HEN, HEH'. ILH. IDL

REAL MLL. LAII

DEFALILT YHLUES - TEHP IH C: GAS COISTANT IN L-ATMM, AGMLE

DATH TPEF . R. O.0.0.08205,

C.ALL GET IHAME. 1., X. 1)

HPP:

IF I HAME, NE, AHPPOP, GO TO 290

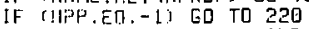

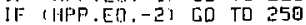

UR TE (NOUT, 320 ) NPP

[I, ?II NTINEE= L. MPP

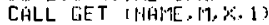

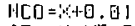

IF (IIHHE.EO, $4 \mathrm{H}$ '1OUE) GO TO 10

IF (HAME.EO. AHCPLI) GO TO 30

IF WHIE. EO . AHEPVA] CO TO 50

IF (WATL. ED. AHEWTL) GO TO 70

IF (WHIF.EO. AHEHTW GO TO 90

IF (HAHE. EQ, $4 H L H Y 1 B$, GO TO 110

IF (HAPE.EO. $\triangle$ HUAPR) GO TO 130

IF (HHIE. EO, SHLIILS) GO TO 150

IF IHAHE.ED.4HDEHL) GO TO 170

IF (HAIT. ED. HHTREF) GO TO 190

IF IHAHE. EQ AHR , GO TO 200

WP [TE (HEPF, 300 ]

STOP

EOH'T IHUE

MOLECULFR UJE [GHT

I0 $20 \quad I=1 ., 1 C 0 M P$

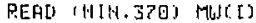

LIR I TE (NOUT, 360 ) (1WII)

CDNHTINE

Go TD 218

COHTIHUE

\section{LITLII HEAT CAPACITY}

$\mathrm{HCP}=+\mathrm{HCO}$

[ID $40 \quad I=1 ., 4 C D M$

REA[ (NIN, 370) (CPL $([]),, J=1, N C D)$

UIR ITE (NOUT, 360) CCPL[C, JJ, I=L, NCO)

COHTLHUE

1009 


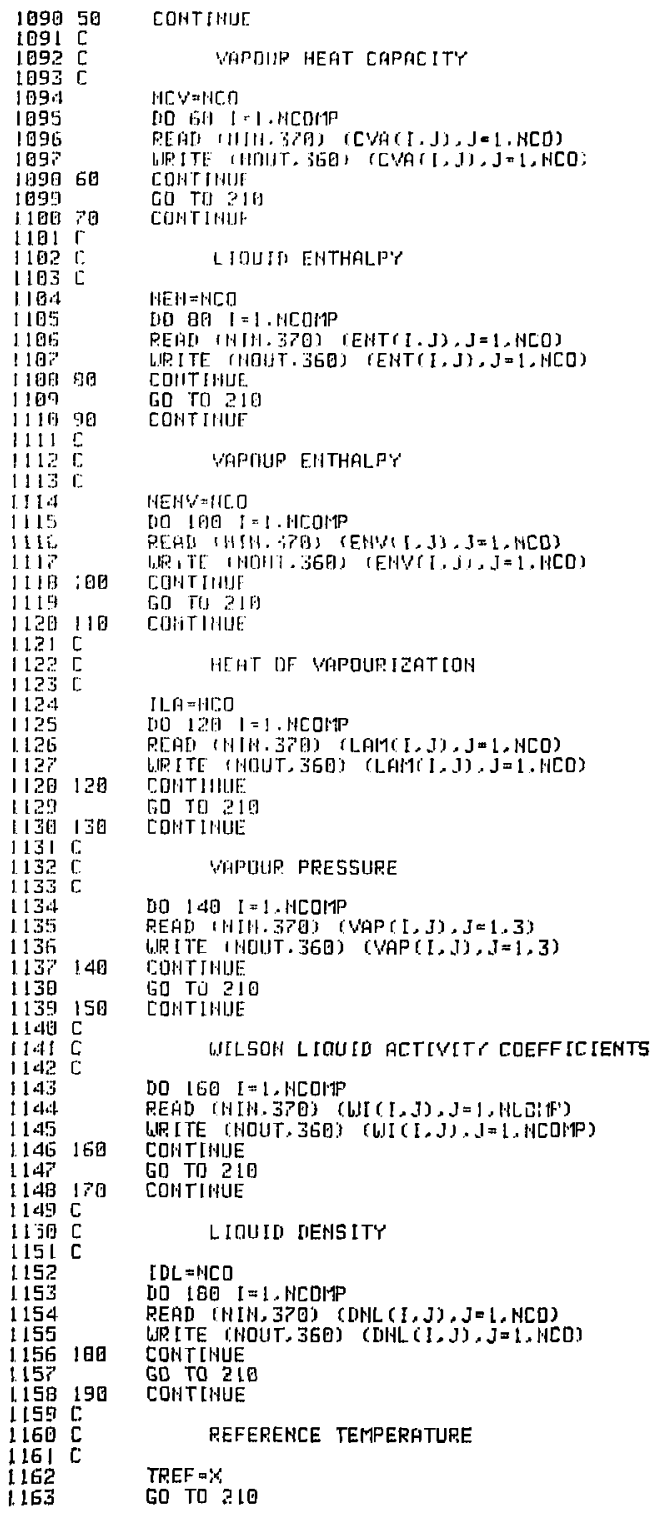




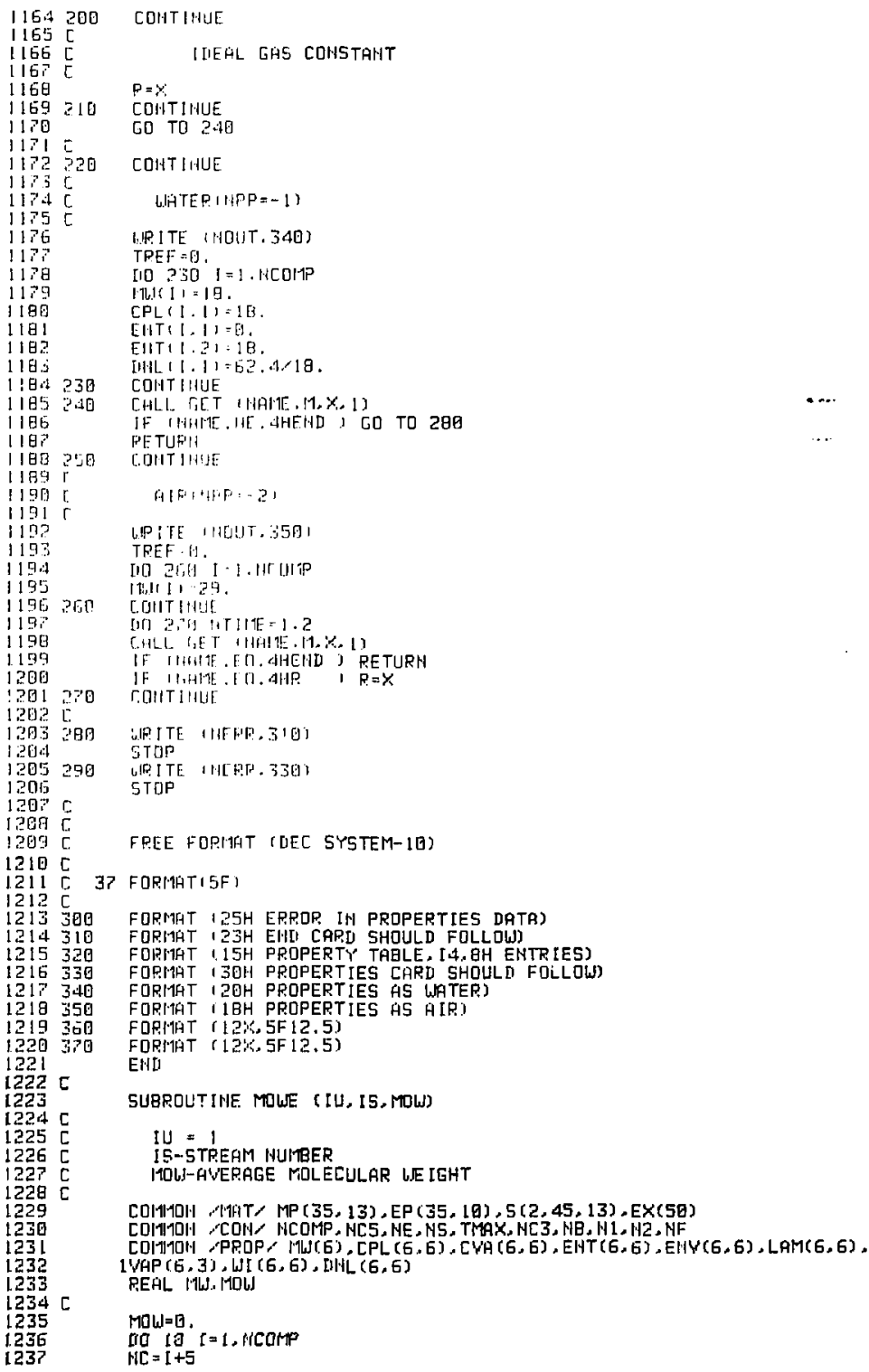




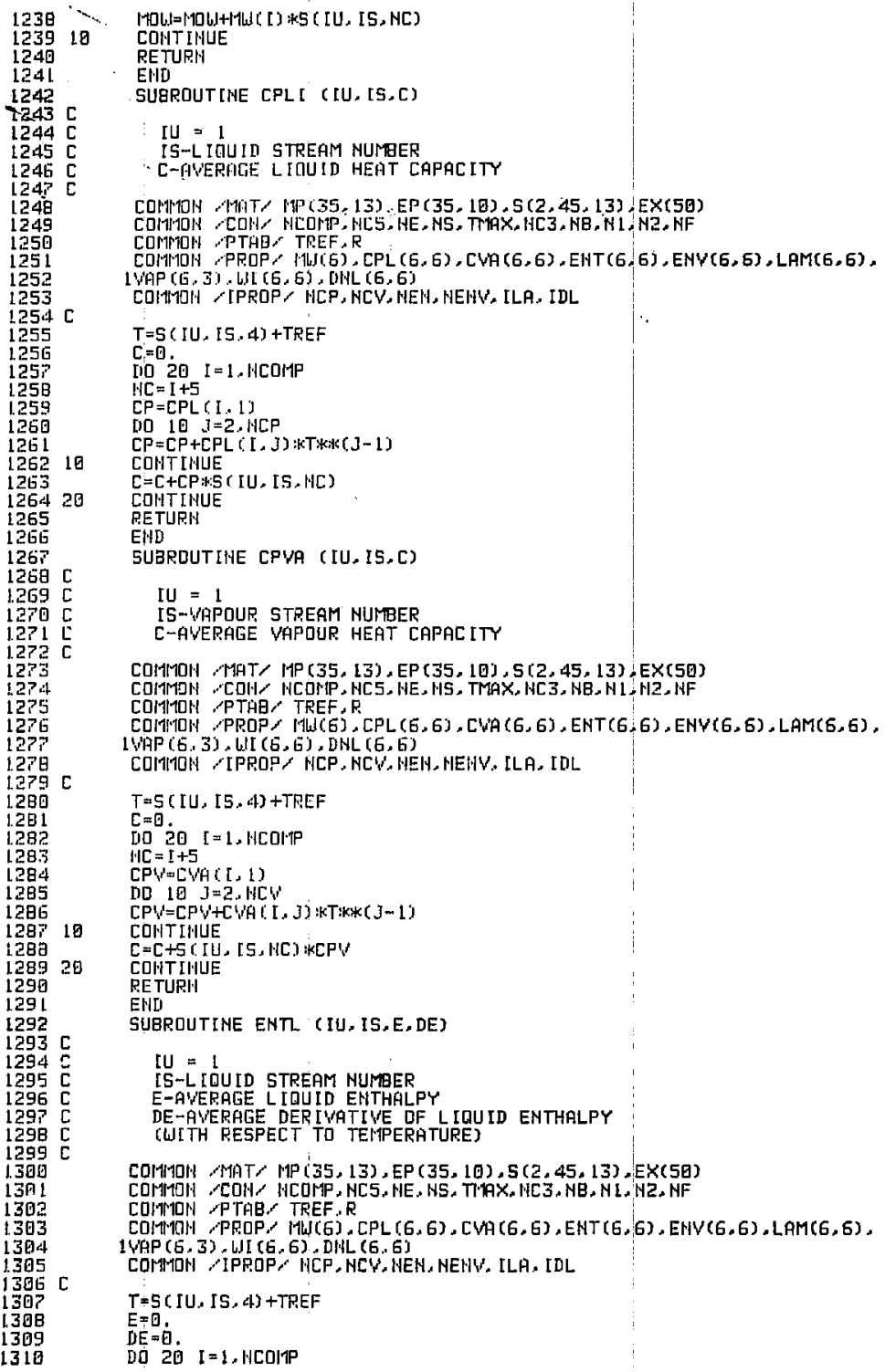




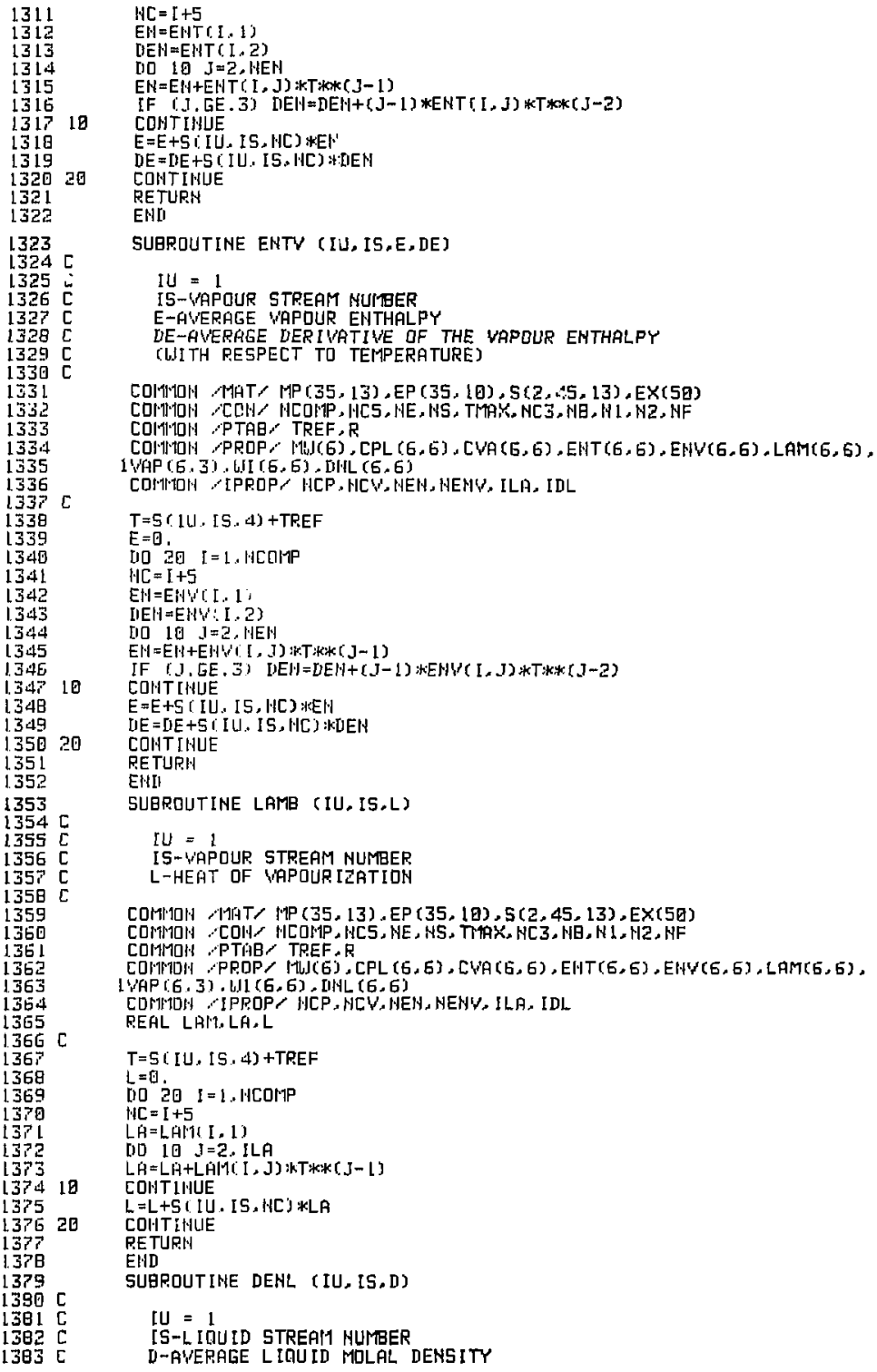




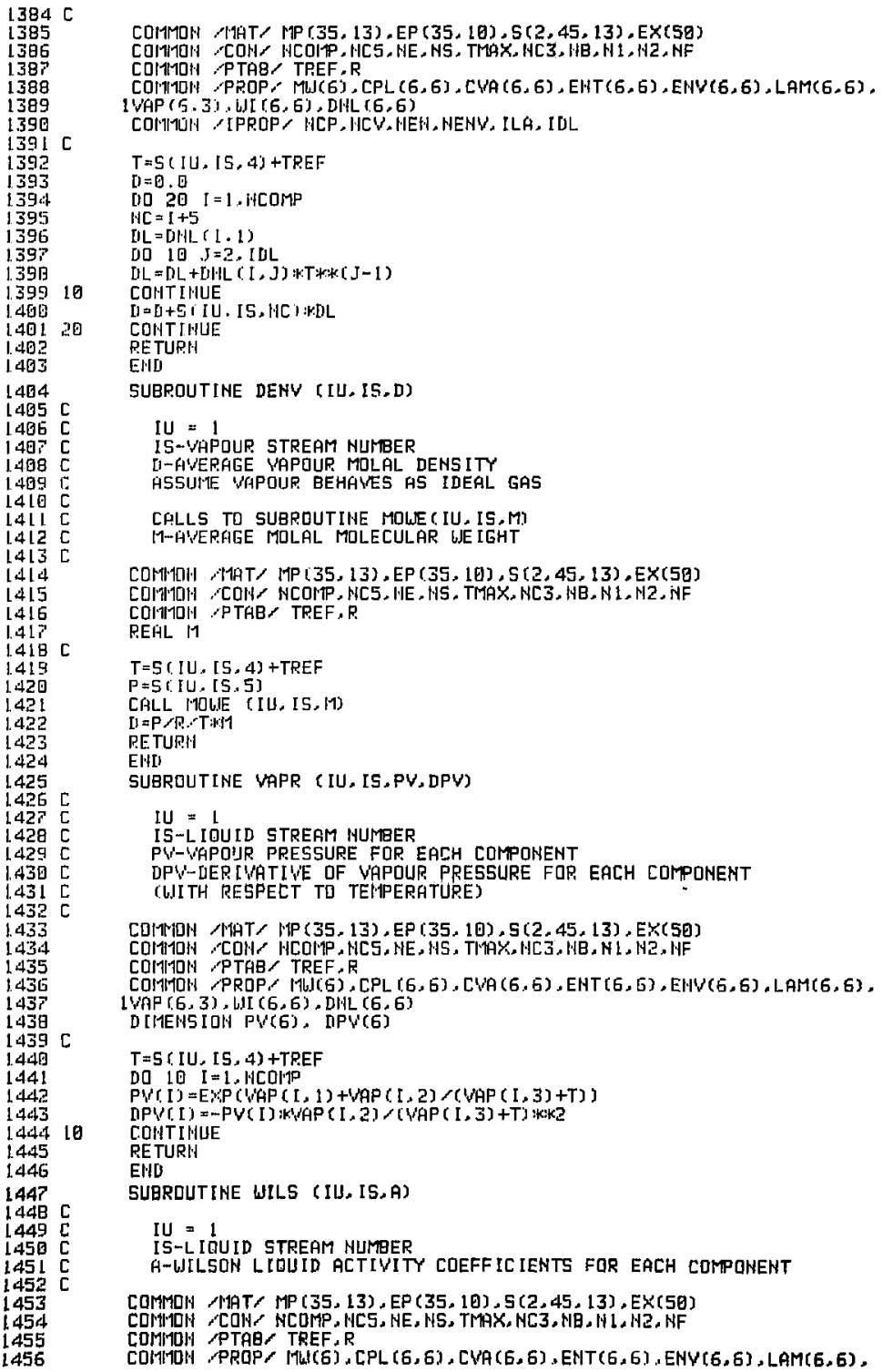




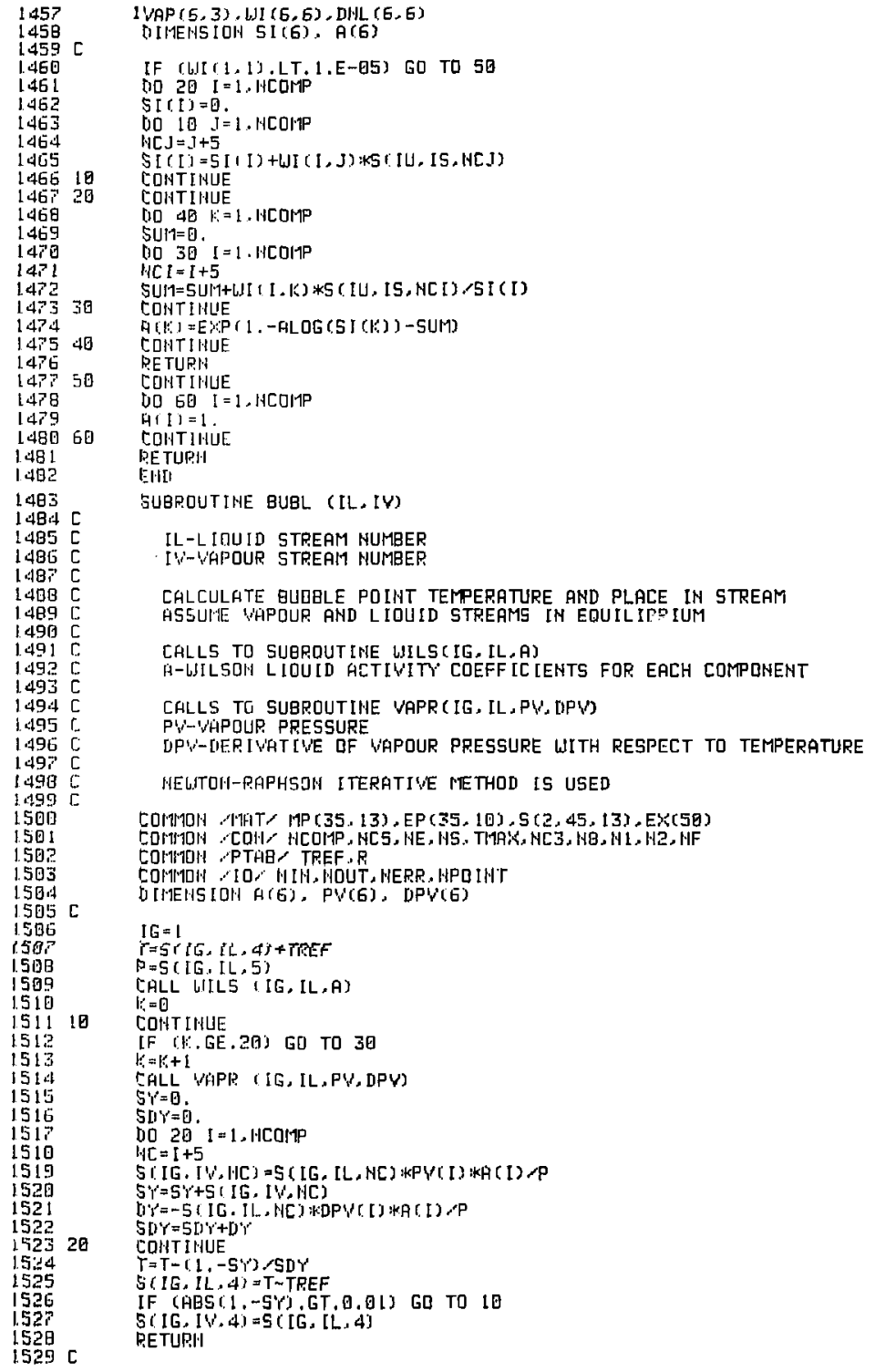




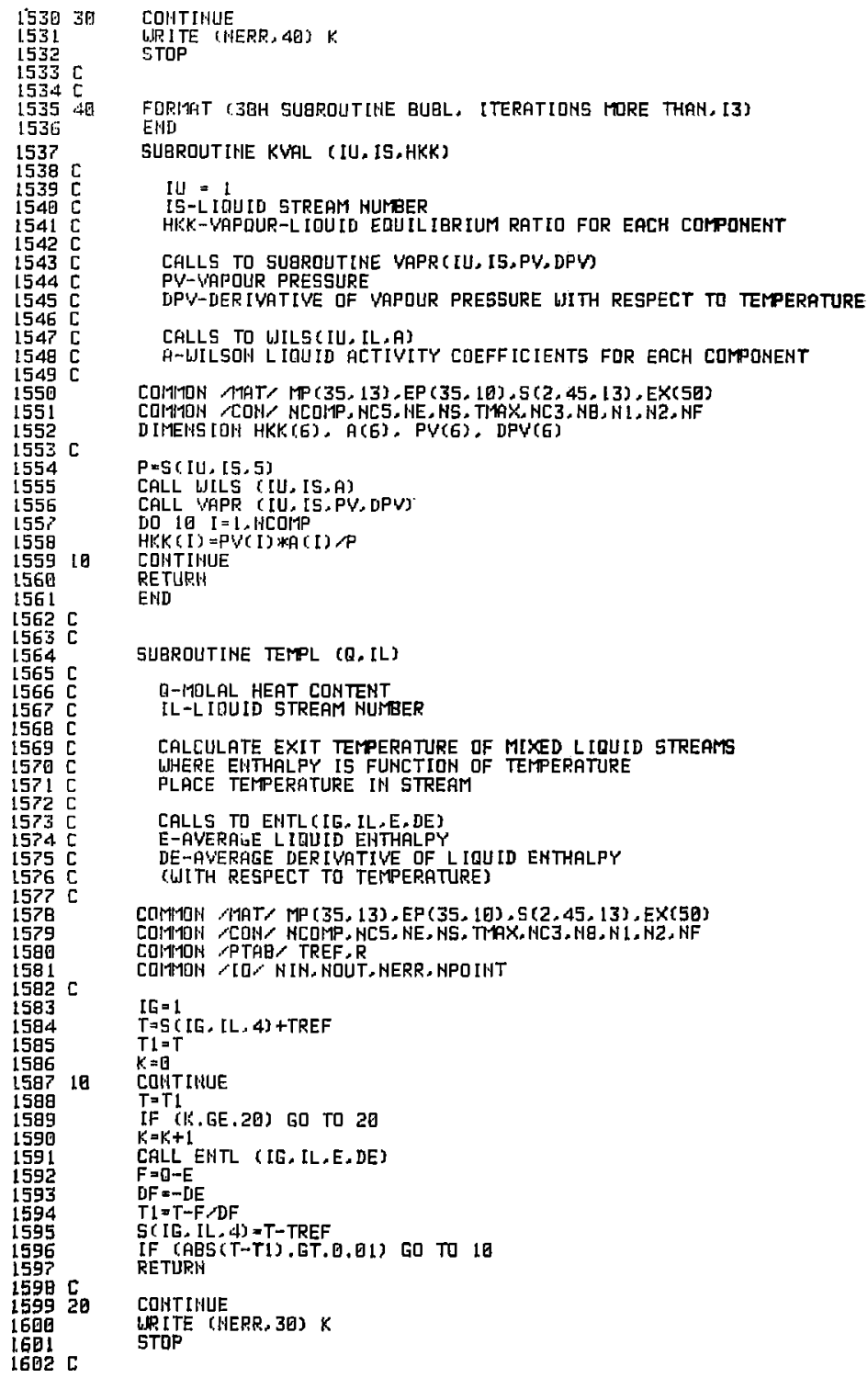


1603

I604 30

1605

1606

1607

1608

1609

$1610 \mathrm{C}$

1611

1612

$1613 \mathrm{C}$

1614

1615 C

$1616 \mathrm{C}$

$1617 \mathrm{C}$

$1618 \mathrm{C}$

1619

1620

1621

1622

1623

$1624 \mathrm{C}$

1625

1625

I62?

1628

L629

1630

1.631

1632

1633

1634

1635

1636

LE3?

1538

1639

1640 C

164120

1642

1643

1644 C

1645

164630

1647

1648

1649 C

$1650 \mathrm{C}$

$1651 \mathrm{C}$

1652 C

$1653 \mathrm{C}$

1654 ᄃ

$1655 \mathrm{C}$

I656 C

$1657 \mathrm{C}$

$1658 \mathrm{C}$

1659

1660

1661

1662

1663

1664

1665

1.666

1.667

1.668

1669

LEPQ C

IEPI

1672

1673

1674 C

i.65 C
FGRMAT (39H SUGROUTINE TEMPL, ITERATIOH MTRE THAH, I3)

ENII

SUBROUTINE TEMPV $(A, I V)$

Q MOLAL HEAT CONTEHT

IW-STPEAM NUMGER

CALCULATE EXIT TEMPERATIRE OF MIYED VAPOUR STREAMS

WHERE ENTHALPY IS FUNCTIOH OF TEMPERATURE

PLACE TEMPERATURE IN STREAM

CALLS TO ENTV(LE, [V,E, DE)

E-AVERAGE VAPOUR ENTHALPY

DE-AVERAGE DERIVATIVE OF VAPOUR ENTHALPY

(UITH RESPECT TO TEMPERATURE)

COMMON MAT, HP $(35,13), E P(35,10), 5(2,45,13)$, EX(5)

COHI10N, CON, HCOMP, NC5, HE, HS, THAX, HC 3, HB, NL, N2, NF

COIN1TON PTAB, TREF, R

COIH1DUN, IO, N IN, HOUT, NERR, MPOINT

I $G=1$

$T=S([G, I V \cdot 4)+T R E F$

$T 1=T$

$\mathrm{K}=\mathrm{O}$

COHTIHUE

$\mathrm{T} \pm \mathrm{T}$ l

IF (K.GE, 20) GO TO 20

$K=k i+1$

CAI.L ENT, (IG, IY,E,DE)

$\mathrm{F}=\mathrm{Q}-\mathrm{E}$

$\mathrm{DF}=-\mathrm{DE}$

$\mathrm{Tl}=\mathrm{T}-\mathrm{F}, \mathrm{DF}$

5 (IG. [ $\psi, 4]=T-T R E F$

IF (AES (TI-T).GT.Q.B1) GO TO 18

RETURN

CONT [HUE

LIR [TE I.NERR, 3B] IR

STOP

FORMAT I39H SUGROUTINE TEMPV. [TERATLONS MORE THAN, [3]

END

SURROUTINE TYPEL

SUGROUTLNE STGIDL

DHE IDEAL STAGE FOR EXTRACTION WITH SPEC IFIED

PHASE VOLUTES - SPEC IF IC TO CO-EXTRACTION OF I AND PU

FROM OTHER SALTS - EACH STAGE MAY HAVE ANY HUMBER OF FEEDS

AND EFFLUENTS UP TO FOUR EACH - CODE MAY GE GENERAL IZED

TO EENERAL EXTRACTION DF ANY SYSTEM AHD TO VAR IAELE PHASE

VDLUITES - EXTENSION TO PLI+3 - UtG SEPARATIOH POSSIBLE

COMTON,AHT,MP (35, 13),EP(35, 10), $\$(2,45,15), E X(50)$

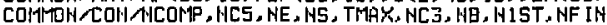

COIH1DH , GERR, JSTART. IME TH, TIME, H, HIMC, EPS, TT. INTTL

COMMON/UIT I IM. HMP

COMMOH IO NIIY, HOUT, NERR , HPO [NT

REAL LIII, LOUT, K'V, KEXT

IHTEGER OUTPRO, OUTSTO, OUTPRA, OUTSTA

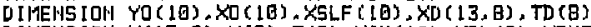

DIMENS IOH K(13,8), W(

D [IEENSION YIN (10), YOUT (10), D [SCO (10), YIDEAL (10),P (B), DERX(10)

DIIENSIOH YO(13,日), XXX(13,日), TRAHS (19)

EQUIPHENT PARAMETERS

1 - PLI HOLDUP, E 


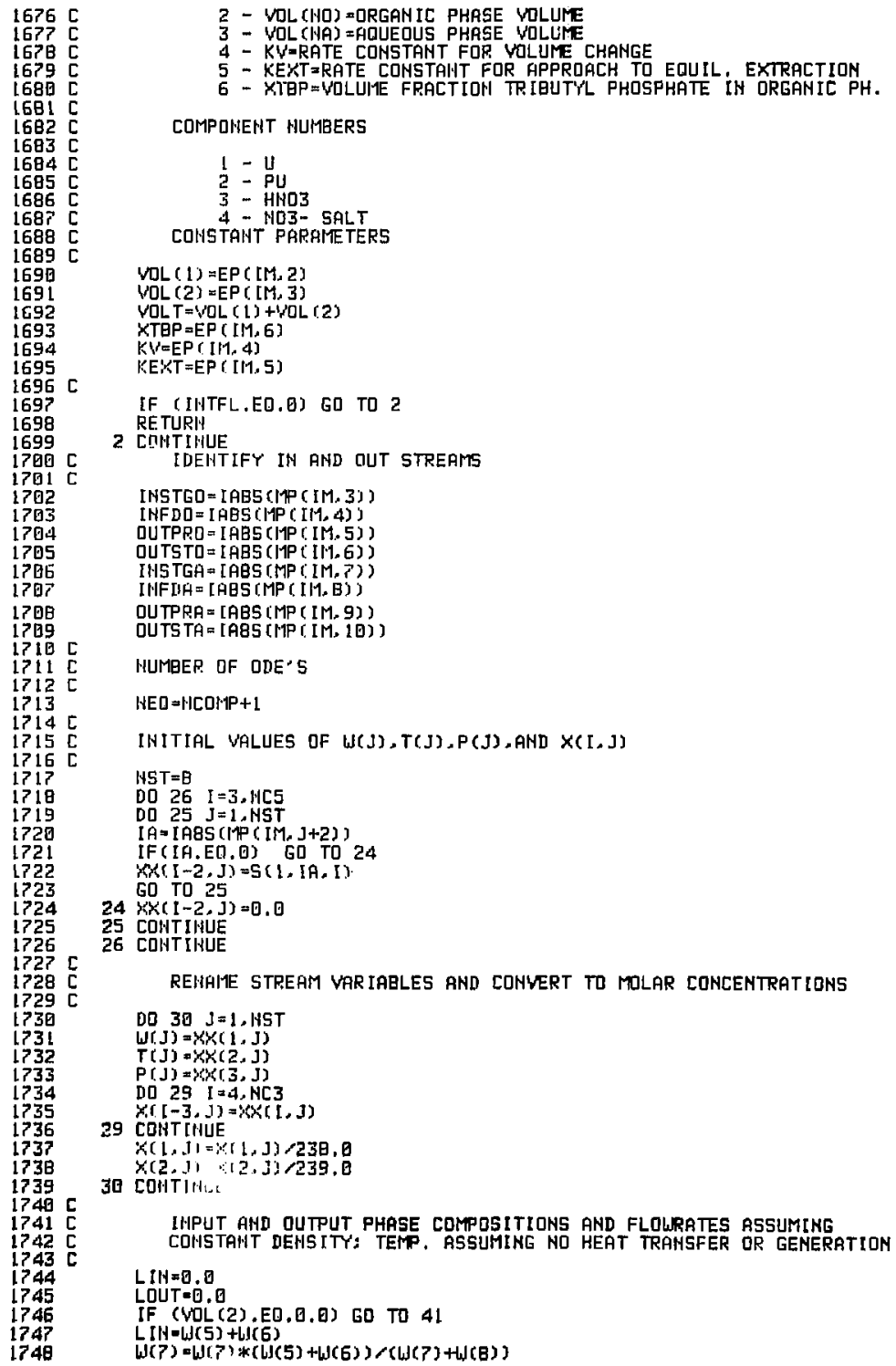




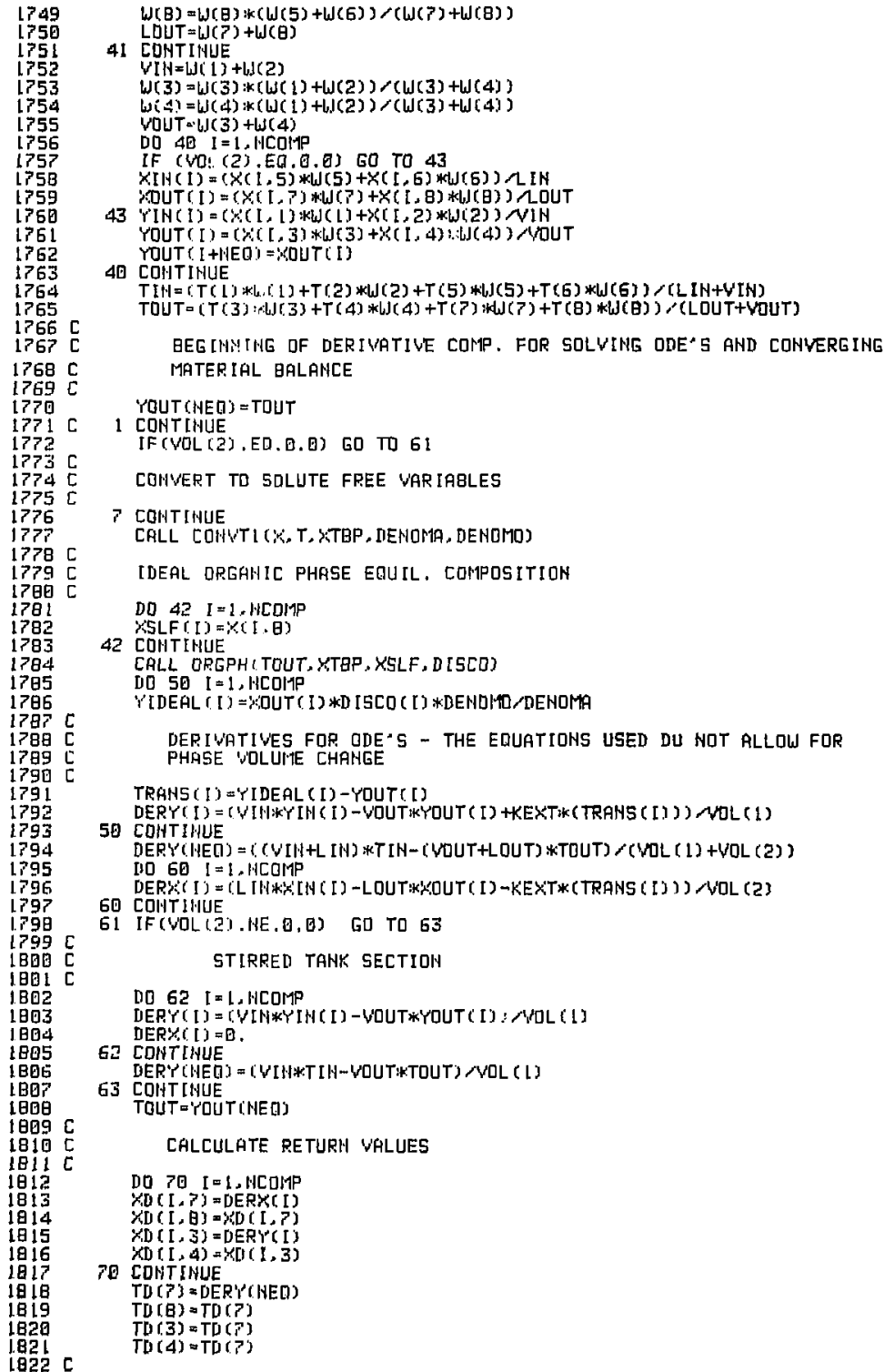




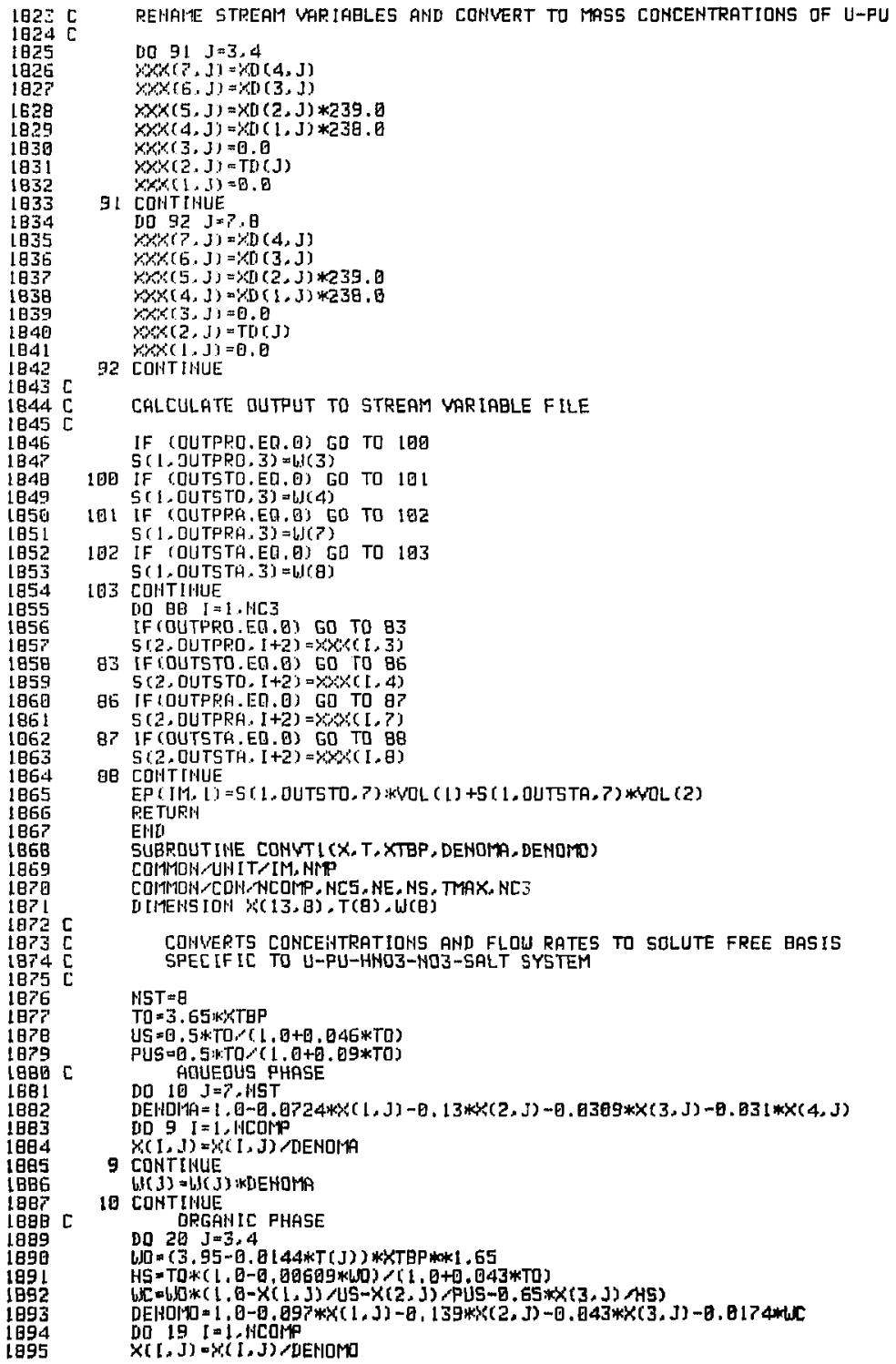




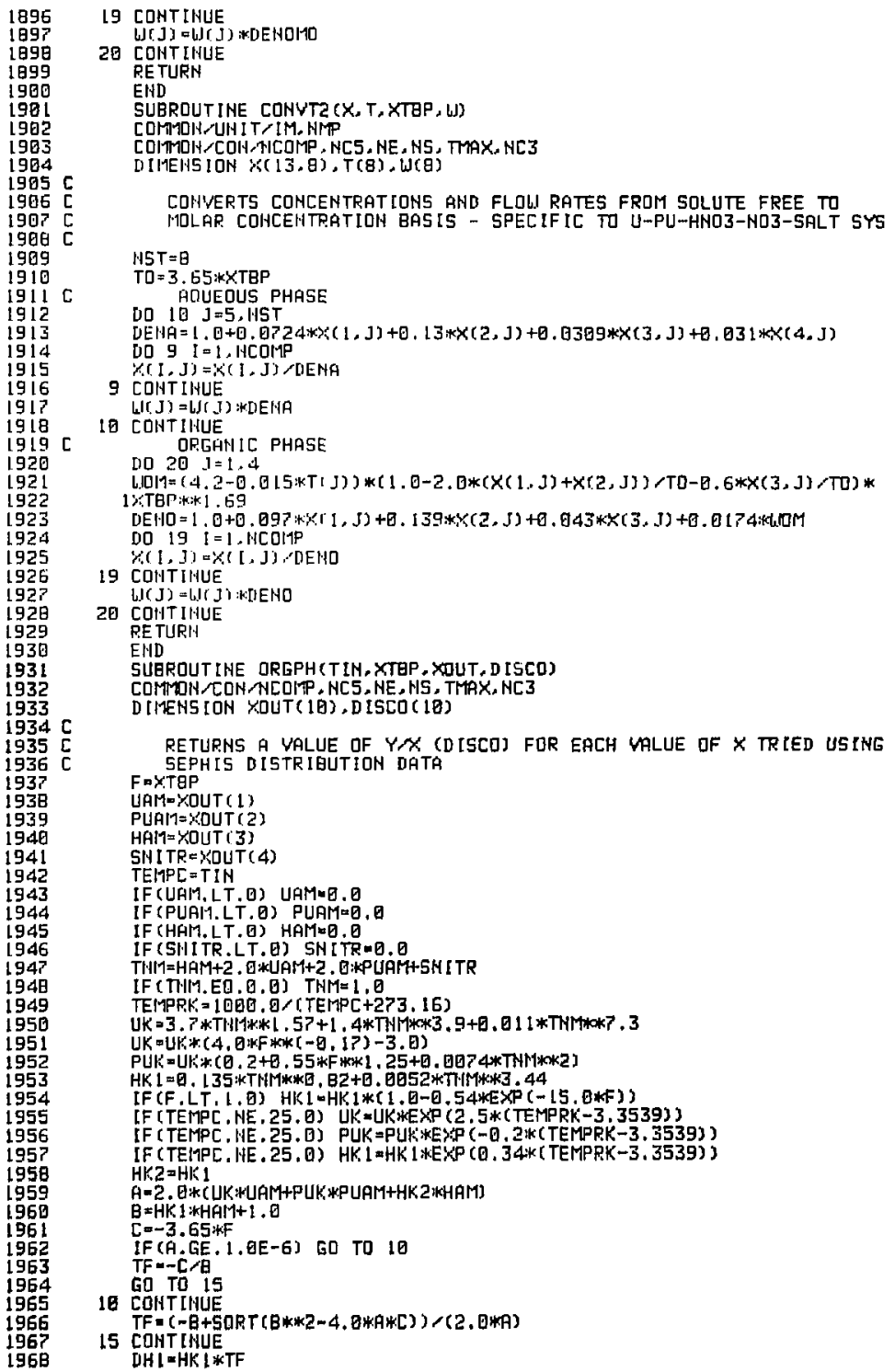




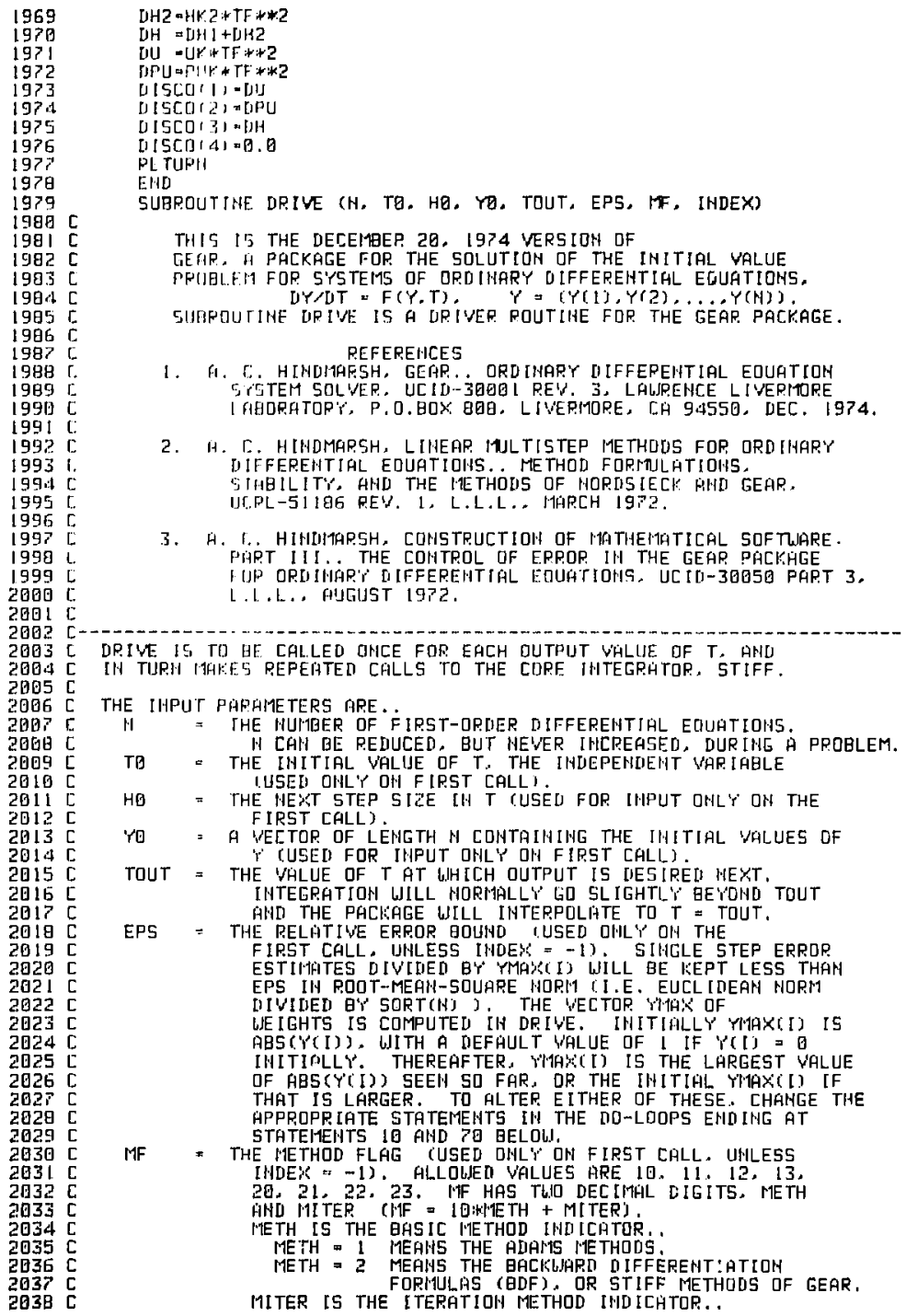




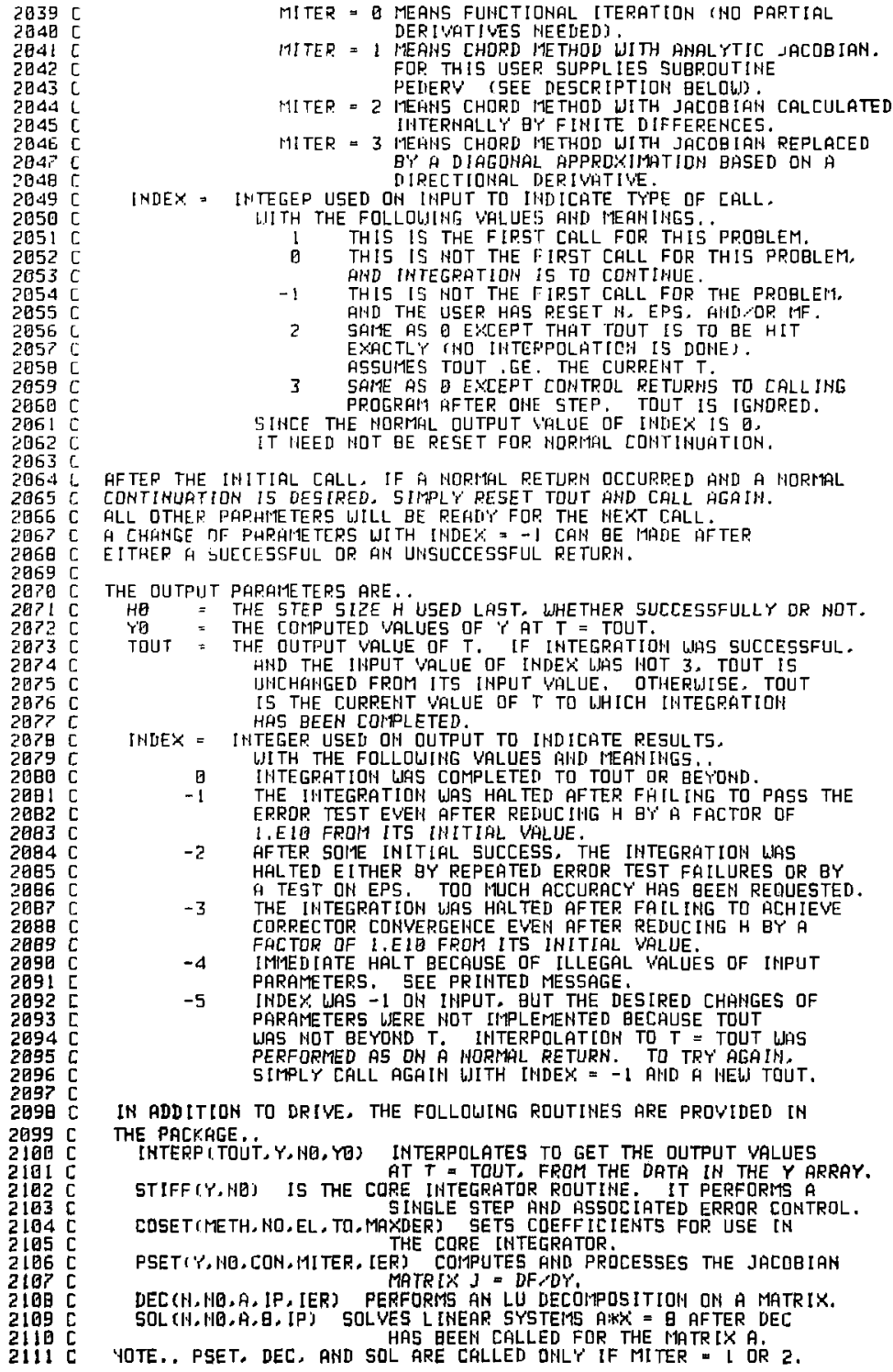


$211 ?$

21131

2114

2115

2116

गा1?

211日

21!95

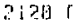

닌.

$2122 \mathrm{f}$

21256

$2124 \mathrm{c}$

?12r.

2lat L

Pl?

2द्य 1 .

$2124 \mathrm{C}$

? 1301.

21311

21320

213.j 1,

213.41

213'

21361.

213i

?191.

ए।

?) 4 il.

?1.41 1

$214=1$.

21430

21441.

2145 C

त) $1.46 \quad \mathrm{C}$

$214 i^{\prime}$

$2148 \quad$ C.

2149 1.C+

2150 1..

$2151 \mathrm{C.}+$

$215 \%$ C.

$2153 \mathrm{CC}$

$21548+$

$21550 .+$

2156 C.C.

2157 ГC.

2. $158 \mathrm{Crt}$

$2159 \mathrm{CC}+$

$2160[\mathrm{C}+$

$2161 \mathrm{CC}+$

2)

$2163 \mathrm{CC}+$

$2164 \mathrm{CC}+$

2165 C.C+

या65 CLt

2. $6{ }^{7} \mathrm{CC}+$

2.160 [C+

2169 CC+

2170 $\mathrm{CC}+$

2IP1 Cट+

2172 CC+

2.173 C.C

2. 174 CC+

2175 CC+

2175 C.C+

$2 \operatorname{lip} C[+$

$2.178 \mathrm{CC}+$

$2179 \mathrm{CC}+$

$2180[C+$

IBI $\mathrm{CC}+$

$21 \mathrm{~B} 2 \mathrm{CC}+$

$2183 \mathrm{CC}+$

3 (日) $C \mathrm{CC}+$

2105
THE FOLLOUIHE, ROUTIHES RRE TO BE SUPPIIED B.T THE USER.

UIFFUHIH, $T, Y$, YDOT, CDIPUTES THE FUHCTIDH YUOT a F(Y,T), THE PIGHT-HAHE SILE DF THE D.D.E. HERE 'Y FHD 'YUOT APE VECTOPS DF LEHGTH N.

PELEPYII, T, Y,PU,HQJ COHPUTES THE H GY H JACOBIAH MATR[X OF PAPTIAL DEPIWATIVES. AHID STORES IT IN PD AS AH HO BY HO ARPAY. PUI [.JI IS TO BE SET TQ THE PHPTIAL DEPIVIHTIVE DF 'TIOTII) LITH PESPE[T TO 'IJJ. PELIERV IS CALLED DHL'Y IF MIIEP = 1 . DTHERGISE A DUHTY PRUTIHE CAH BE SUBSTITUTEL.

THE DIHEWSIOHS IN THE FOLLOLIIHG DELLAPATIOHS HPF SET FOP A MA:IIMIS DF PA EDUATIOHS. IF THE PACKAGE IS TO USEL FOR A LARGER WHLUE OF $M$, THE JIMENSIDHS SHDULD BE HCPEASED ACCORDIHGLY THE

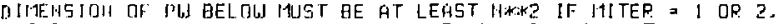
BUT EAH EHE PI EUCED TD II IF MITEP =3. QP. TO I IF IIITEP $=0$.

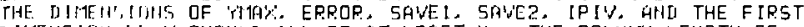
EIIIEHGIOH II $\%$ SHOULII GLL BE AT LEAST H. THE COLUINA LEHGTH OF

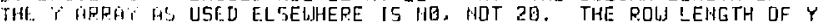
CAII HE PI IIITI [ FPUM 13 TO 6 IF METH $=2$.

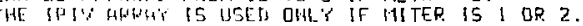

THF COI'WU HI DCK GEARG CAN BE ICCESSEI EXTERHALLY G\% THE USER IF JIESIRI II. IT CONTAIHS THE STEP SIZE LAST USED (SULCESSFULLY), THE UPDI I HE T USEI I GUCCESSFIJLLYI. THE HIIHEP DF STEPS TAHEN

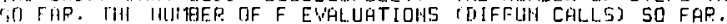

HHI THE HIIHI!: UF JACOBIAHA EUALIJHTIOHS 50 FAR.

IH THE FIIIIISINIT [IFTA STATEIEHT, SET.

ISPIJHIH THE UIIT POUHUOFF OF THE IHCHIHE, 1.E. THE SMALLEST

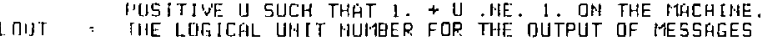
IMP. IHG THE IHTEGRATIOH.

LALJPEYICE L I'VERMORE LABORATORYY

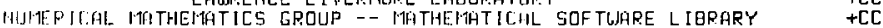

\begin{tabular}{l} 
+CD \\
\hline
\end{tabular}

CLASS DHE ROUTINE: DRIVE

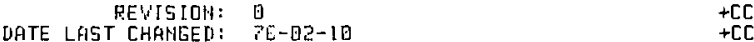

RELEASE STATUS: UNL IIIITED

EACH CLASS OHE ROUTIHE HAS GEEN THOPOUGHLY TESTED GY NITG GND MEETS CERTAIH DIICUIMENTATION HHD PROGPAMMIHE STHMIUARDS.

AT LEHST DHE COHSUL TANT IS HWALLABE TO AHSLER OUESTIOHS AND RESPOND TO REPOFTED ERROPS OR INAUERUACIES IN H CLASS DNE POUTINE.

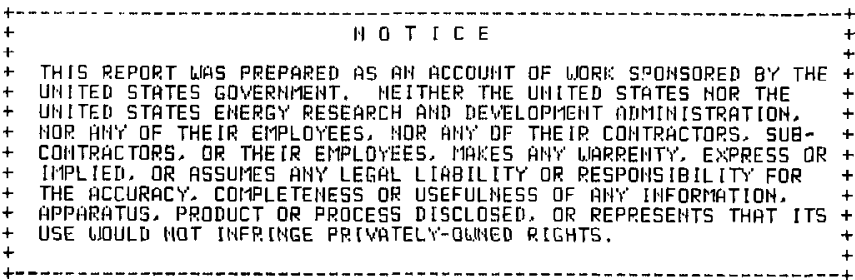

PLEASE REPJRT AHY SUSPECTED ERRORS IH THIS ROUTIHE IPTIEDIATELY TO HMG, EXT. 3049 . 3329, OR 328日. 


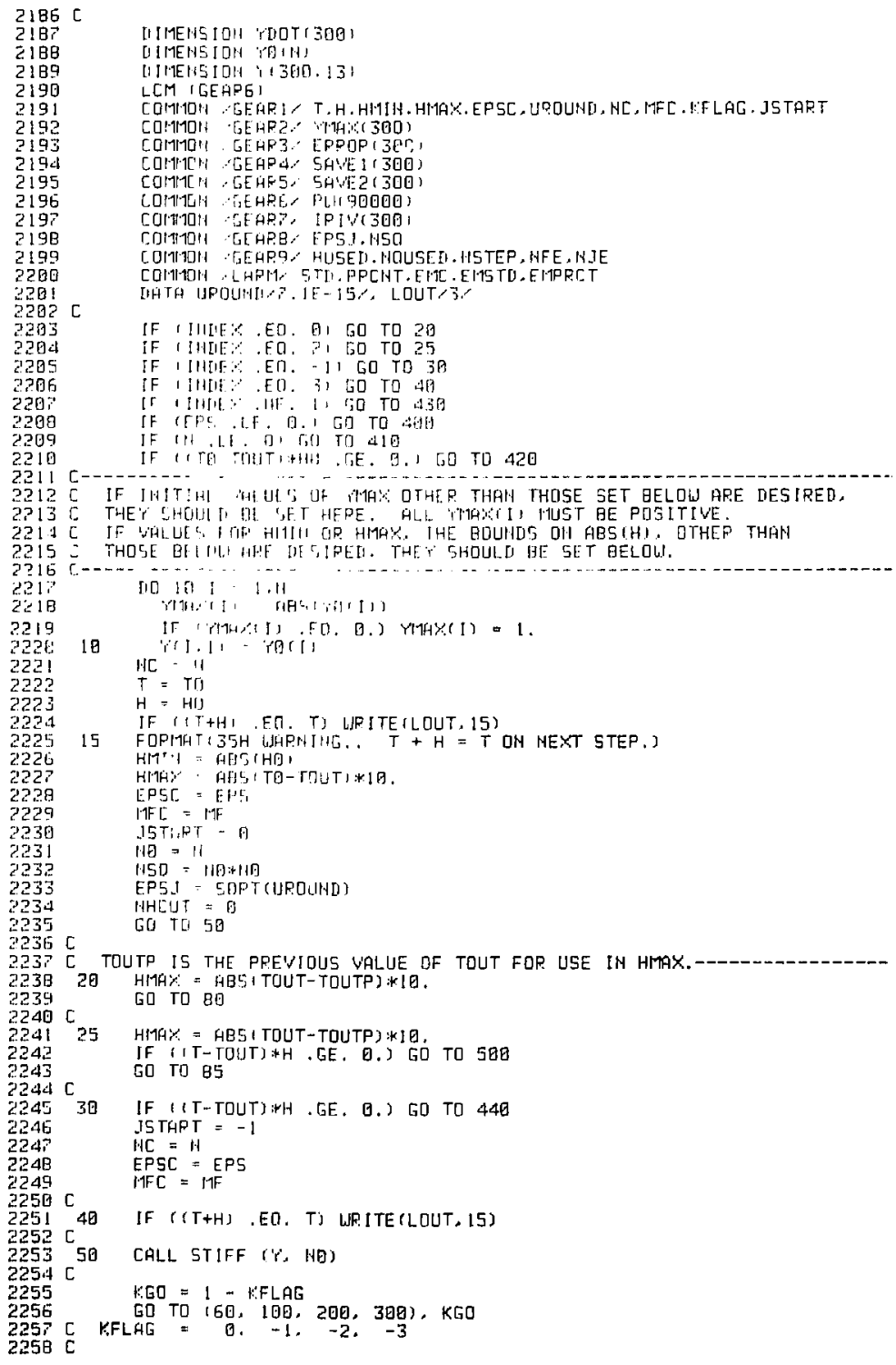




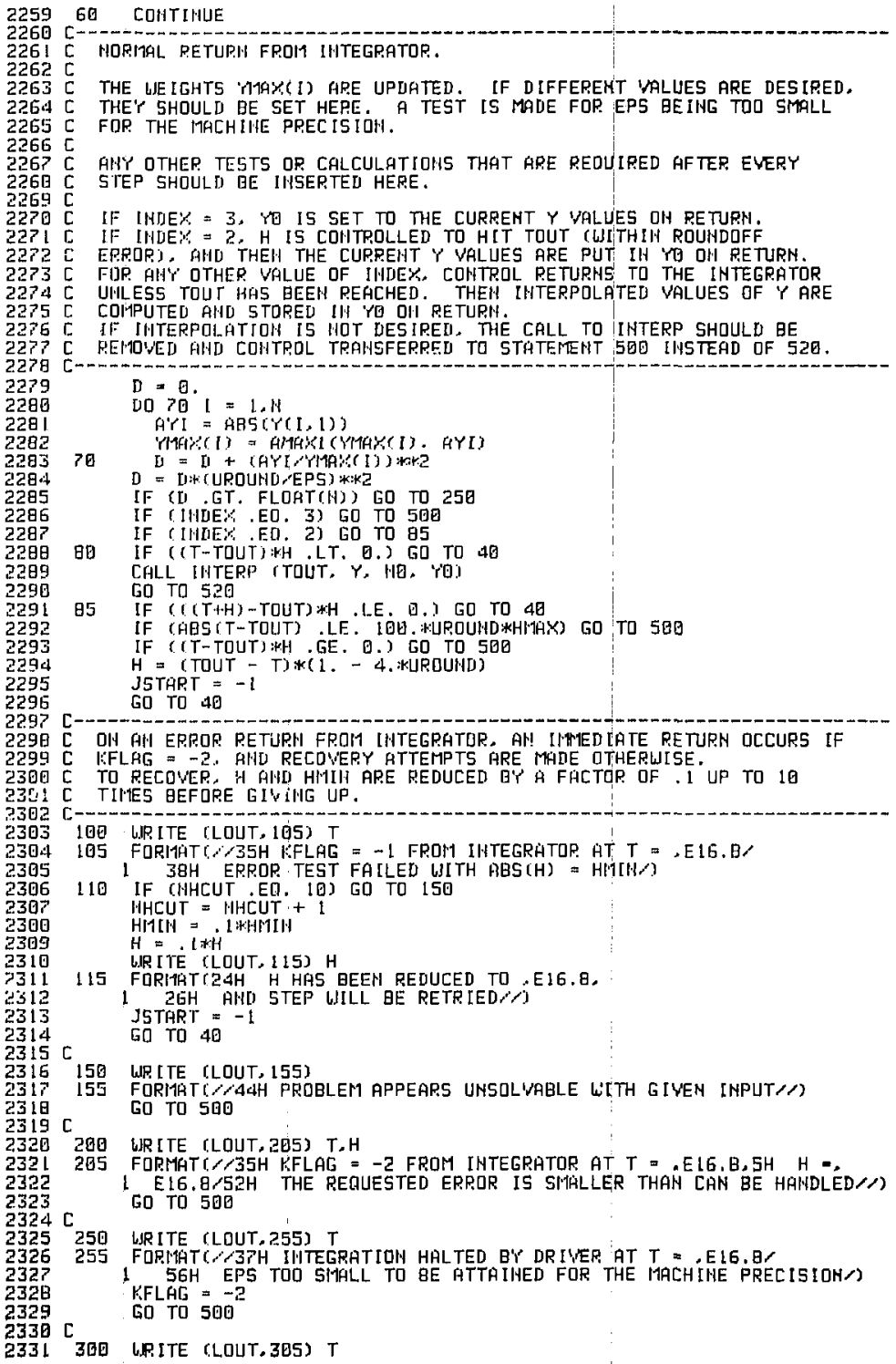




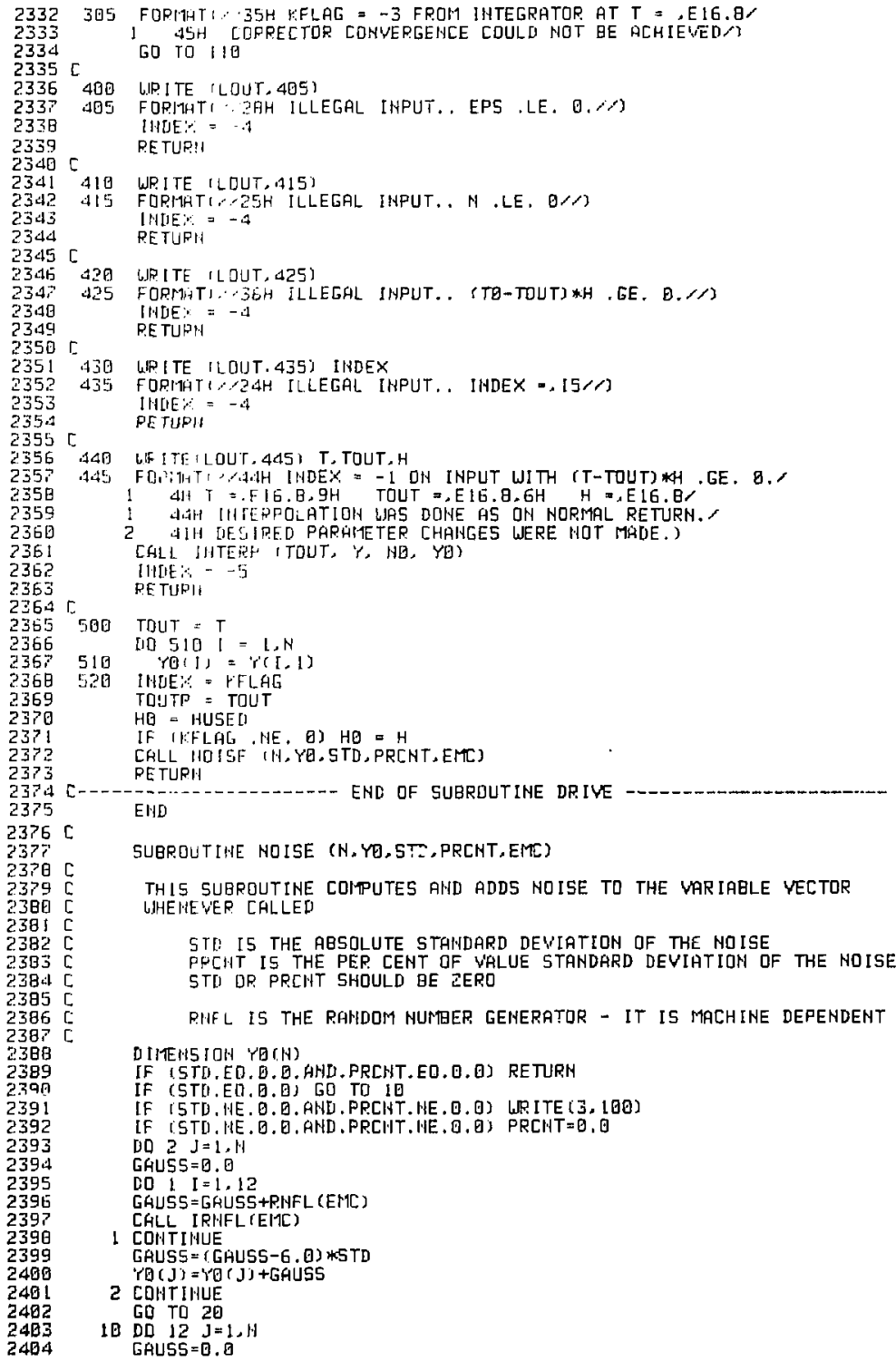




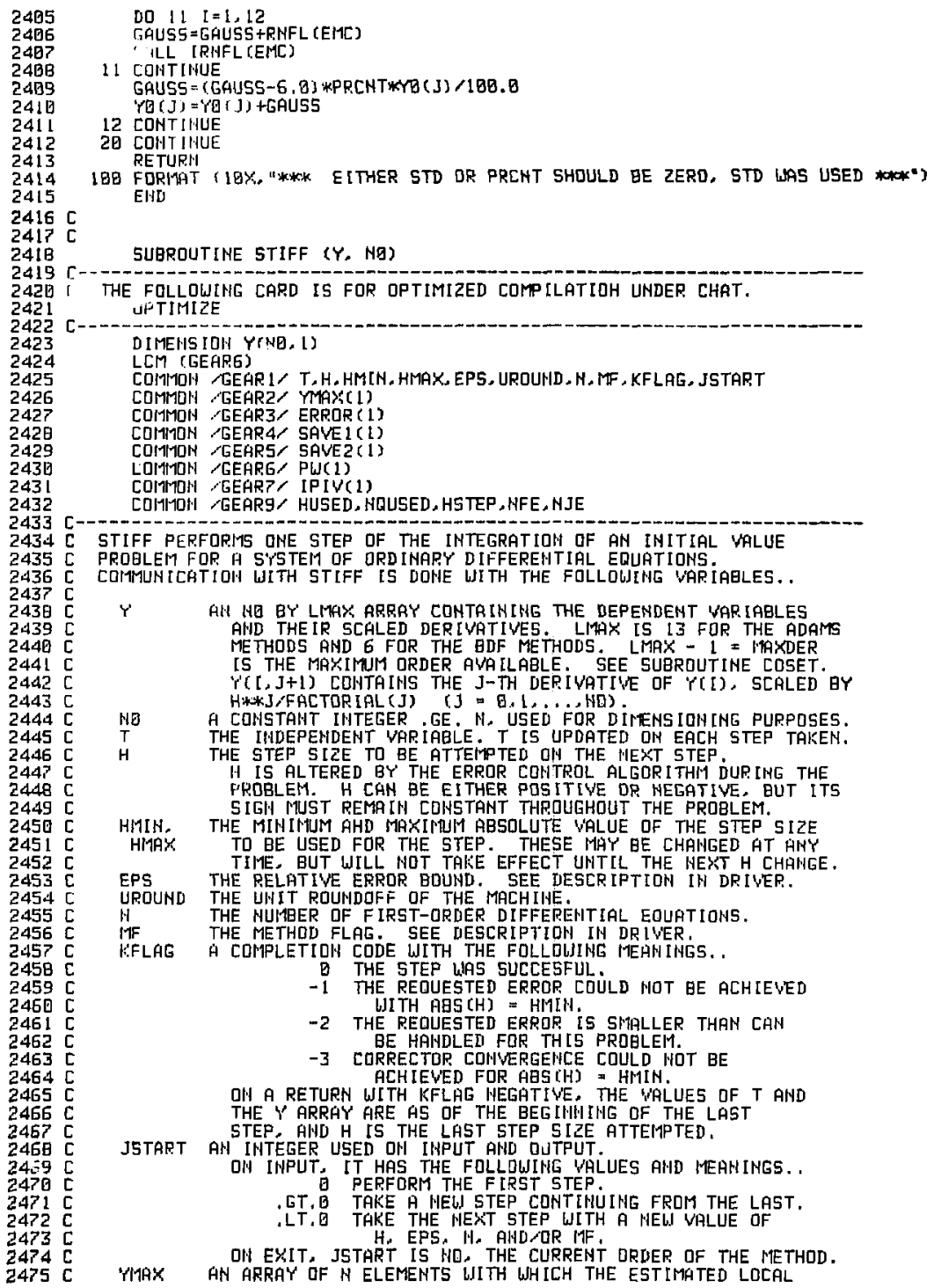




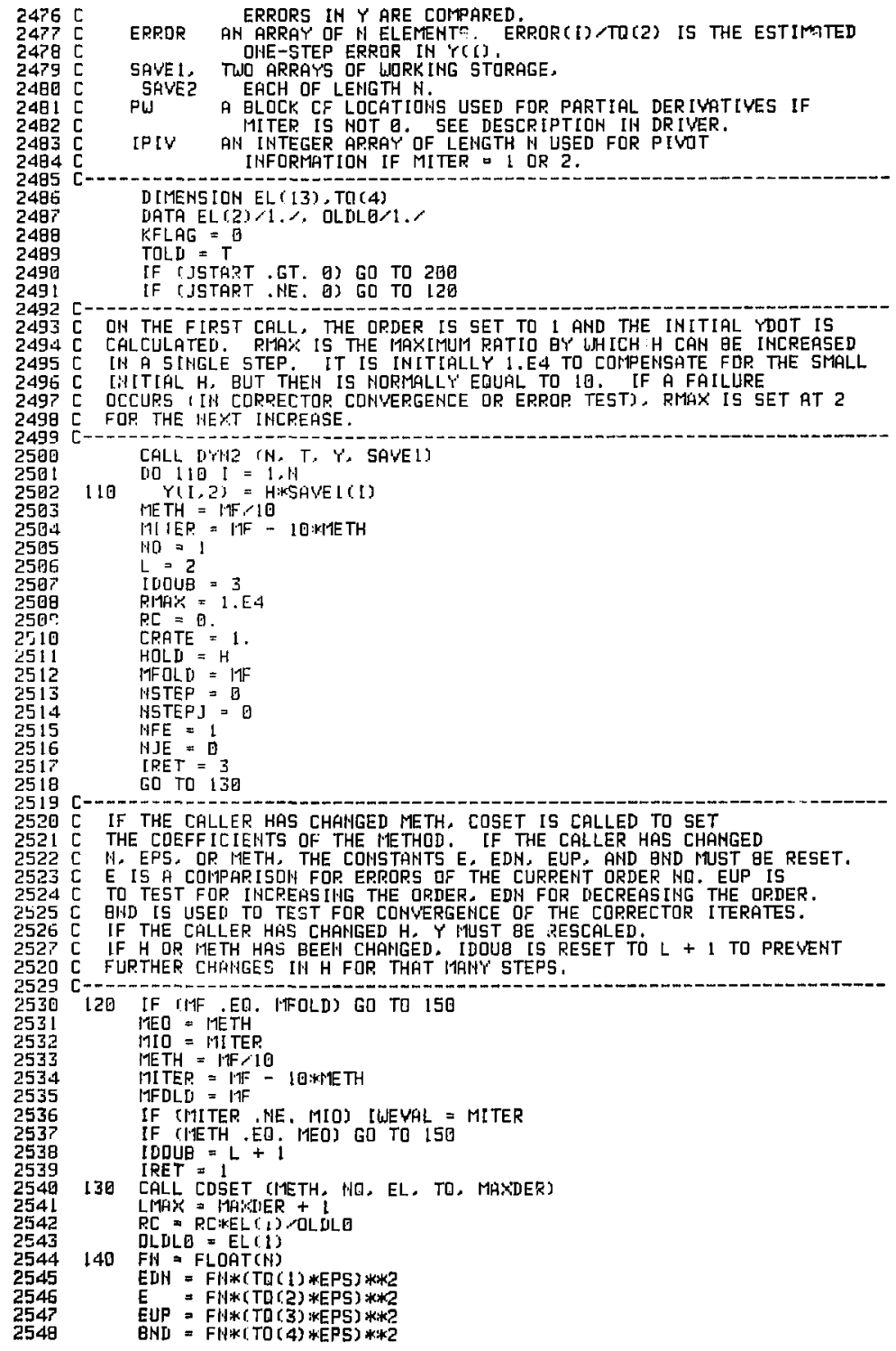




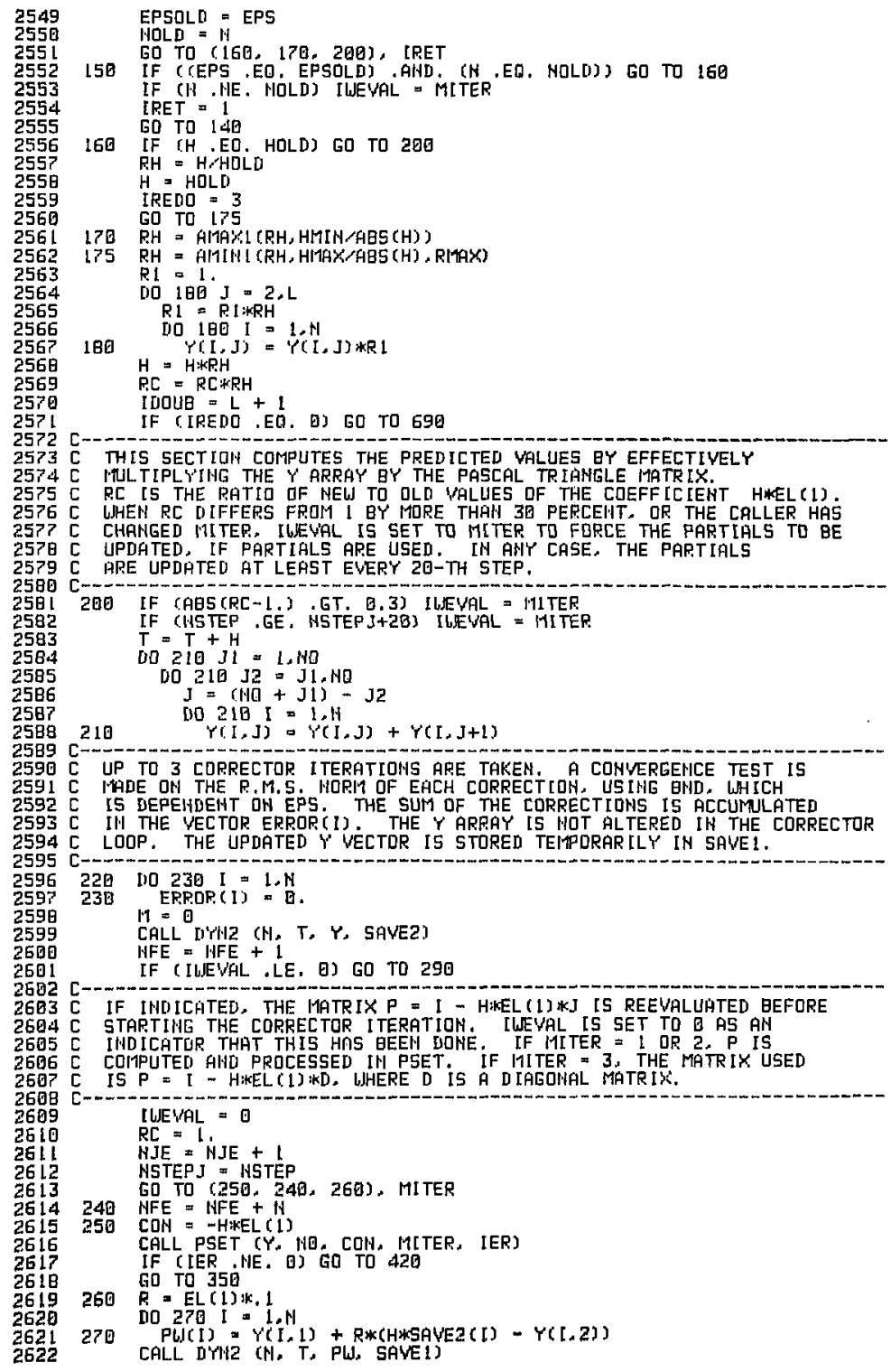




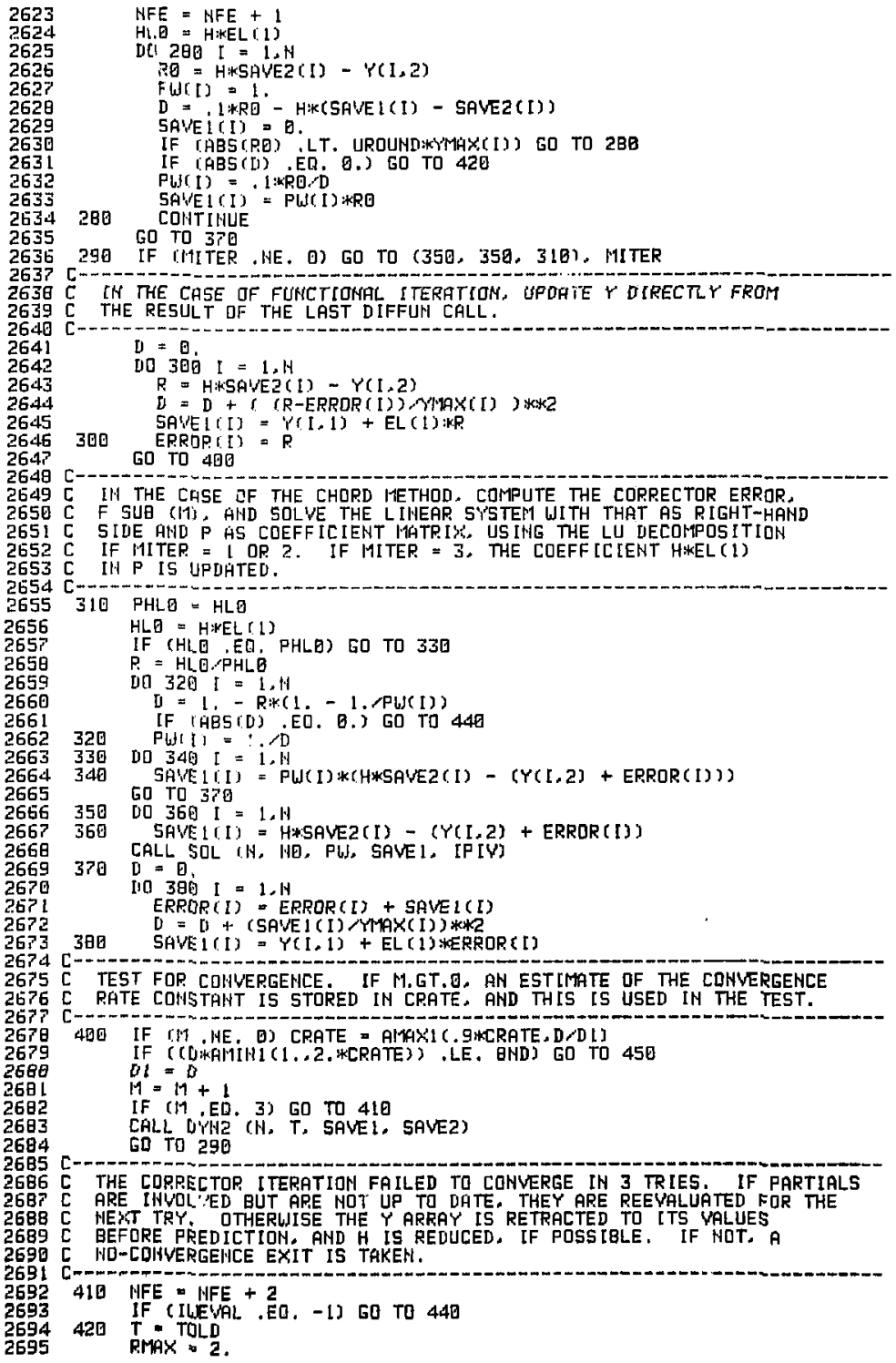




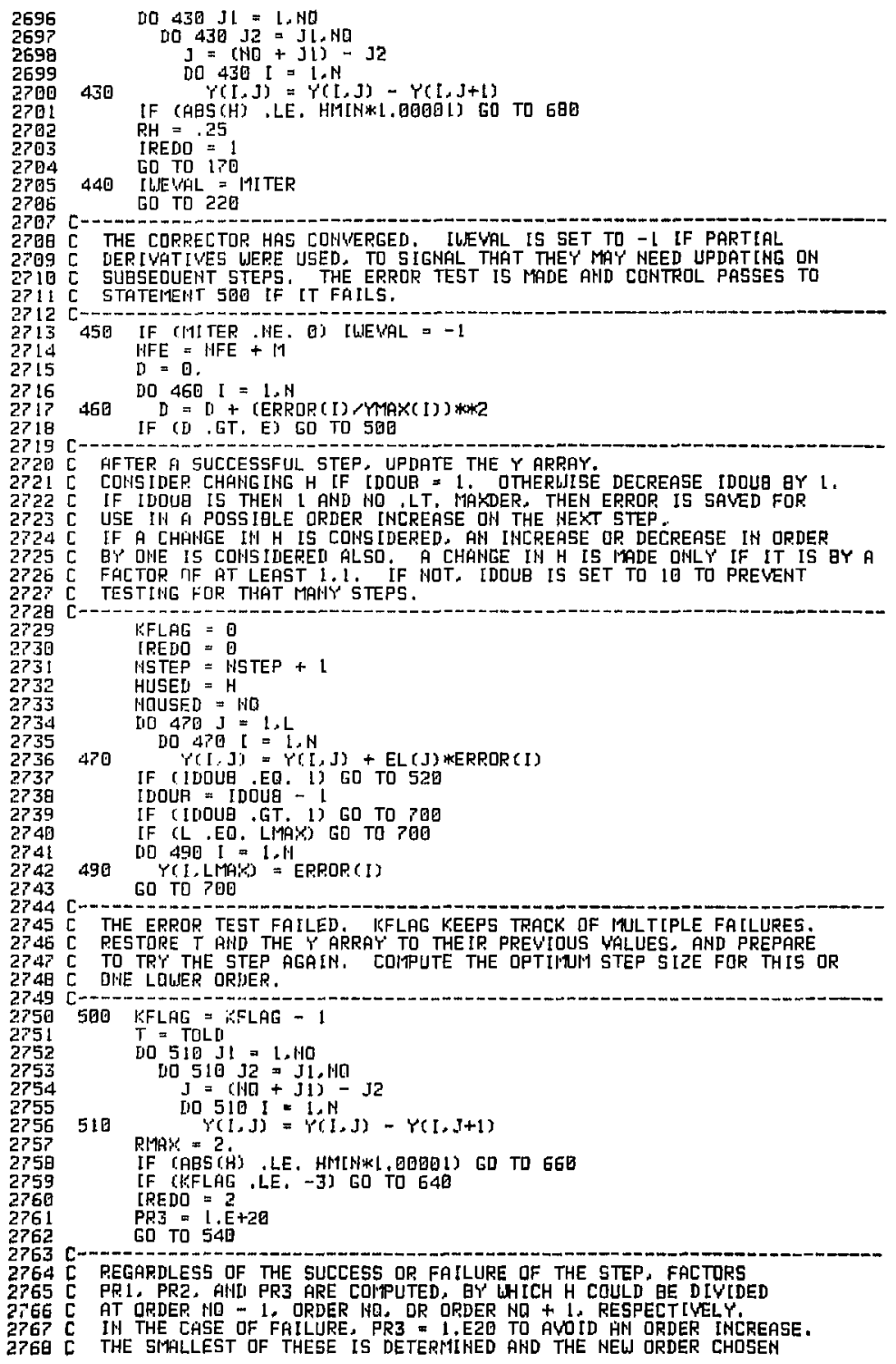


2769 C ACCORDIHELY, IF THE QRDER I5 TO BE IHCREASED, WE COHPUTE DNE

$27 P G$ C AIIDITIOHAL SCALED DERIVATIVE.

2771

2772

2773

2774

2775

2775

2)?

2P?日

27>9

2PBO

27비

2792

2783

2794

2>日5

27日6

27日7

27 B日

2789

2790

2791

2792

2793

2794

2795

2796

2797

2798

2799

2000

2801

2802

2803

2804

2005

2806

2807

2日日 $C$

520 PR3 $=1, E+20$

[F (L ,EQ, LMAY) GO TO 540

$\mathrm{DL}=0$.

DO $530 I=1, N$

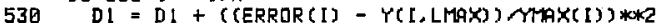

ENDZ $=.5, F L D A T(L+1)$

PR3 3 ( (D L EUP)**ENG3)*L.4 + L.4E-5

540 ENQ2 $=.5 /$ FLOAT(L)

PR2 * ( (D/E) * *ENGI)*1.2 + 1.2E-6

$P R 1=1 \cdot E+2 B$

IF (NQ EQ. I) ED TO 56日

$D=0$.

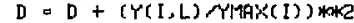

EHQ1 $=.5$, FLOAT (NO)

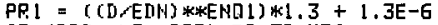

560 IF (PRZ . LE, PR3) EO TO 5PB

IF (PR3 .LT. PRL GD TO 590

GO TO 590

570 IF (PRZ .ET. PRL) GO TO 5BE

NEWQ $=$ NG

$\mathrm{RH}=1 ., \mathrm{PR} \cdot \mathrm{Z}$

ED TO 620

$5 B O$ NELO $=110-1$

$\mathrm{RH}=1, \mathrm{PR}$ :

ED TO 620

NELSO $=\mathrm{L}$

$\mathrm{P} . \mathrm{H}=1 . / \mathrm{PRJ}$

IF (P.H .LT. I.I) GO TO ELO

DO $600 I=1, N$

$Y([$. NEWD+1) * ERRDR (I) *EL (L) /FLOAT(L)

GO TO 630

610 IDOU日 $=10$

E2Q IF (IKFLHG .EQ. O) .AND. (RH .LT. 1.1)) EQ TQ 618

C IF THERE IS A CHANGE OF ORJER, RESET HI. L. AND THE COEFFICIENTS.

2809 C IN AH'Y CASE H IS RESET ACCORDIHE TO RH AHD THE Y ARRAY IS RESCALED

2010

2011

2912

2813

2Q 14

2815

2816

2817

2日1日

2819

2820

2021

2822

2823

2 려

2 B25

2026

2027

2B2B

2829

2830

2B31

2832

$2 \mathrm{B3} 3$

2834

2835

2035

2937

2038

2939

2B40

THEN EXIT FROM 690 IF THE STEP LJAS OK. OR REDO THE STEP OTHERWISE.

IF (NEWI .EQ. NQ) GO TO IPB

HO $=$ MEL, ID

$L=14+1$

[R.ET $=2$ EO TO 130

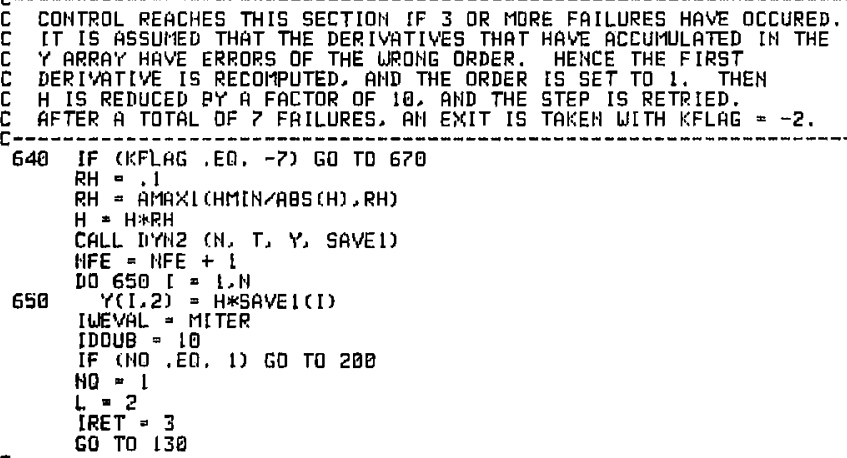


2G4L C ALL RETURHS ARE MADE THROUEH THIS SECTION. H IS SAVED IN HOLD

2042 C TD ALLOU THE CALLER TO CHANGE H OH THE HEST STEP.

2843

2844

2845

2846

2847

2848

2849

2050

2851

2052

2853

2054

2855

2856

2860

660 KFLAG $=-1$

GD TO 700

670 KFLAG $=-2$

GD TO 700

680 KFLAG $=-3$

GL TO 700

$690 \quad R M A X=10$.

PQO HOLD $=\mathrm{H}$

JSTART $=N O$

RETUR

END OF SUBROUTINE STIFF

C

EHI

2日6 1

2862

2863
2864

2日65

2866

2067

286日

2B69

2BP०

2日? 1

2日72

2日73

2日74

2875

2876

2877

2978

2079

2日日0

2日B 1

2882

2883

2884

2895

2日BG

20日7

28日日

2 日9

2091

2892

2893

2894

2895

2096

2097

209日

2899

2900

290 1

2902

2903

2964

2965

2906

2907

290日

2909

2910

2911

2915

SUBRDUTINE PSET (Y. NO, COH, MITER, IER)

C THE FOLLOWIHG CARD IS FOR DPTIMIZED COMILATION UNDER CHAT. DPTIMIZE

DIIENSIOH Y(NO, 1)

LCM (GEAR6)

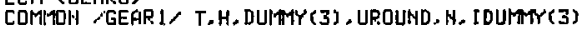

COHMDN /GEAR2, YMAX(L)

COMMOH TGEAR 4/ SAVEL (1)

COMHON /GEARS/ SAVEZ (1)

COIATDH $\rightarrow$ GEARG, PU(1)

COMMDN $/$ GEARP, IPIVCL]

COMMHIT $r$ GEARE, EPSJ,NSG

PSET IS CFLLED EY STIFF TO COMPUTE AND PROCESS THE MATRIX

$P=1$ - H:KEL (1) $*$ J . WHERE I IS AH APPROXIIKATION TO THE JACOBIAN.

$J$ IS COMPUTED. EITHER BY THE USER-SLPPLIED ROUTINE PEDERV

IF MITER = 1 . OP GY F INITE DIFFEREISCING IF MITER $=2$.

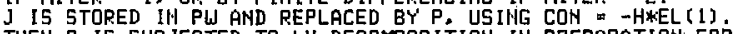

THEW $P$ IS SUBJECTED TO LU DECOMPOSITION IN PREPARATION FOR

LATER SOLUTIOH OF LIHEAR SYSTEMS WITH P AS COEFF ICIENT MATRIX.

IN ADDITION TO VARIABLES DESCRIBED PREVIOUSLY, COMTUNICATIOH

UITH PSET USES THE FOLLOUIHG.

EPSJ = SORT(UREUHD), USËD IH THE HUTERICAL JACQBIAN IHCREMENTS. NSO $=110 * 2 \% 2$.

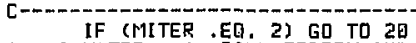

C IF HITER $=1$. CALL FEDERV AND MULTIPL'Y BY SCALAR,

DO 10 I J L.NŚ́

10 PU(C) $=$ PW(I) $*$ CON

50 TO 60

C IF MITEP $=2$, MAKE N CALLS TD DIFFUN TO APPROX[MATE J,

$20 \quad \mathrm{D}=0$.

$30 \quad D=D+5 A V E 2([) * * 2$

RE * ABS (H) *SORT (D) *L . EO3*UROUND

$\mathrm{J}=\mathrm{B}$

U10 $50 \mathrm{~J}=1 . \mathrm{N}$

$r j=r(j, 1)$

$R$. EPSJ*YIMAX(J)

$R$ 口 HIAHXL (R.RO)

$Y(J, 1)=Y(J, 1)+R$

D $=$ CONAR

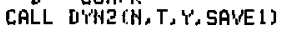

DO $40[D 1, N$

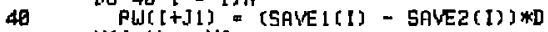

$r(J, L)=Y_{J}$

$\mathrm{JI} J \mathrm{Jl}+\mathrm{NB}$

50 CONT [HUE

C ADD IDENTITY MATRIX,

60 J0" 


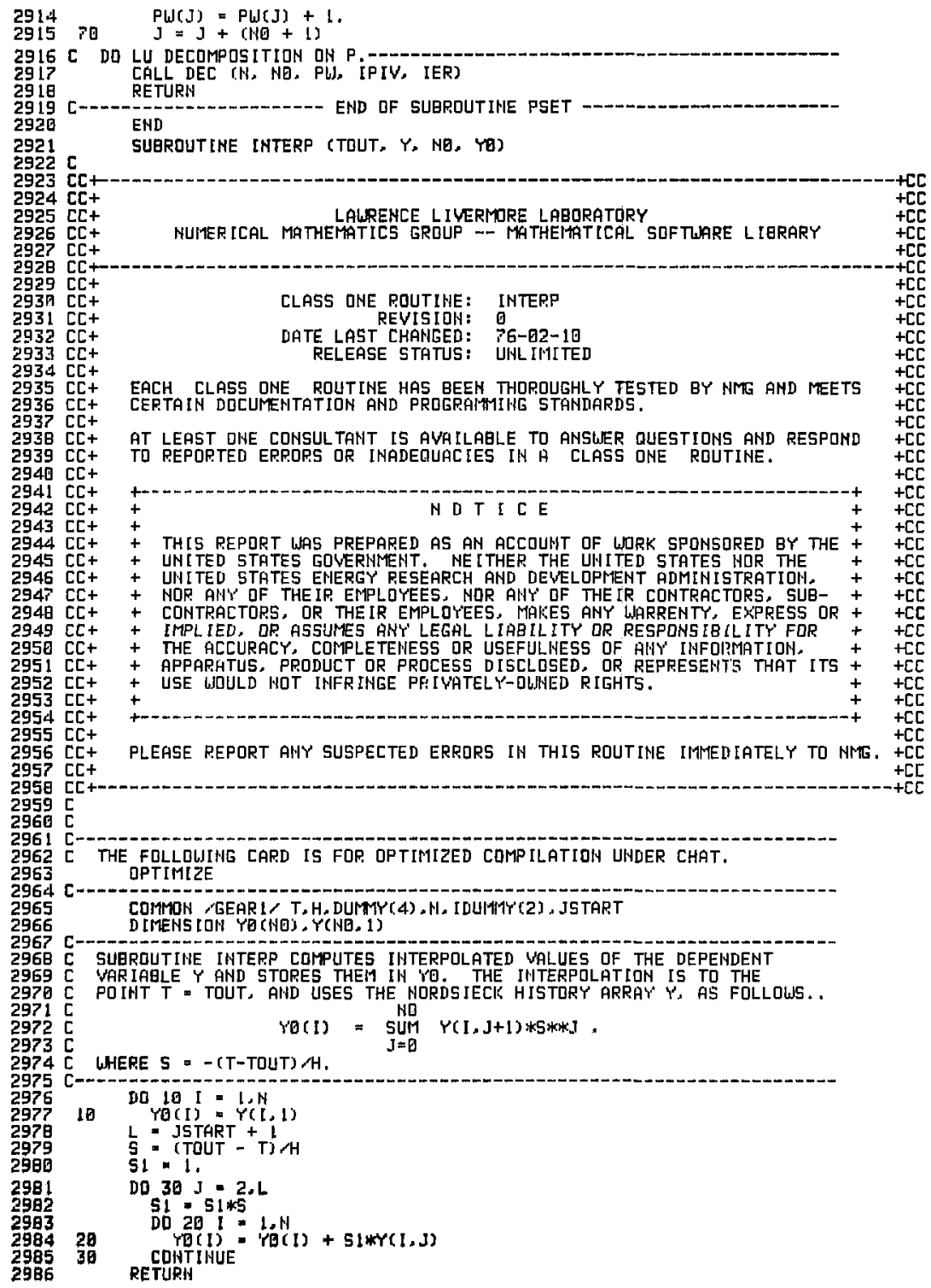




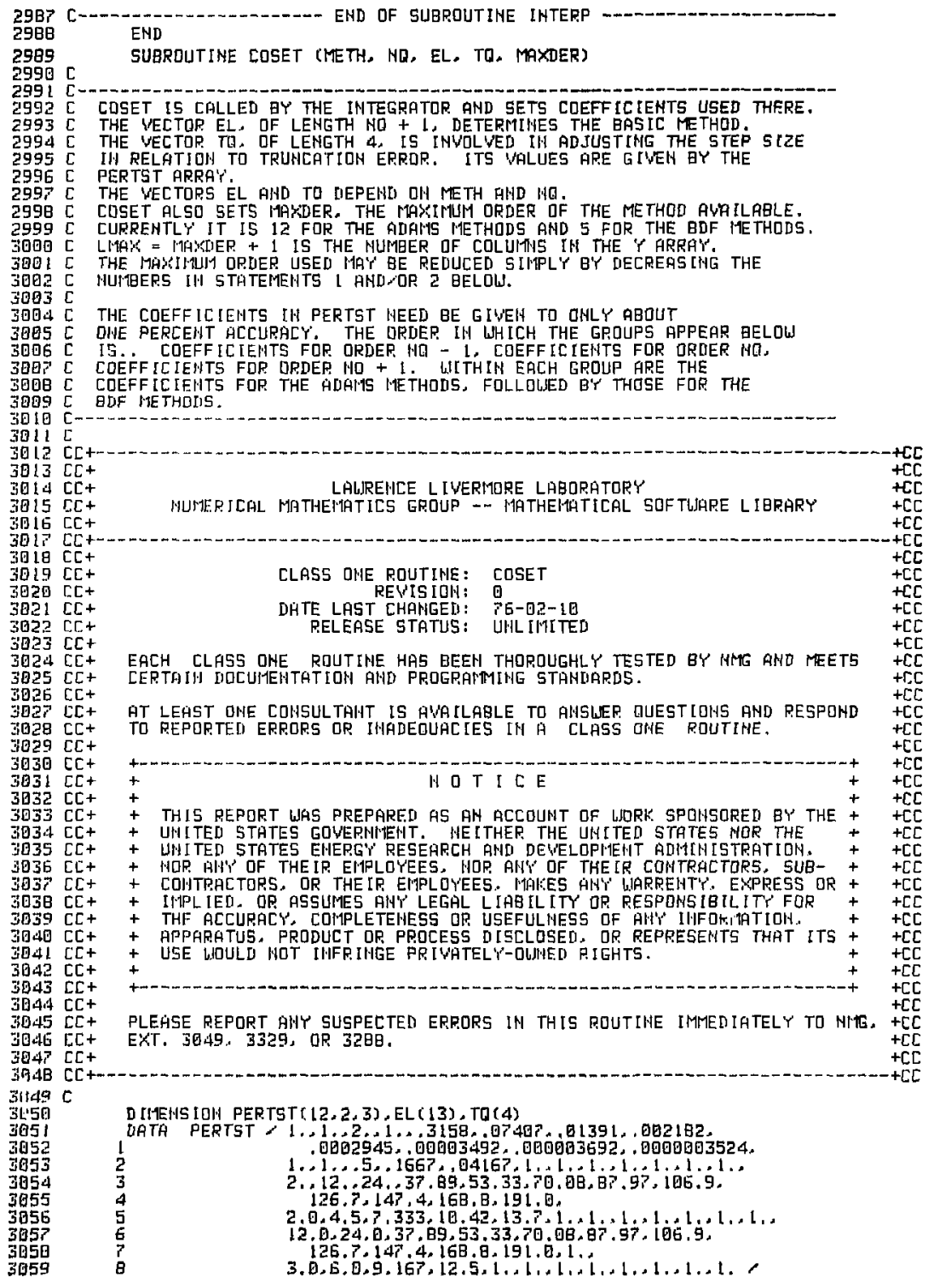




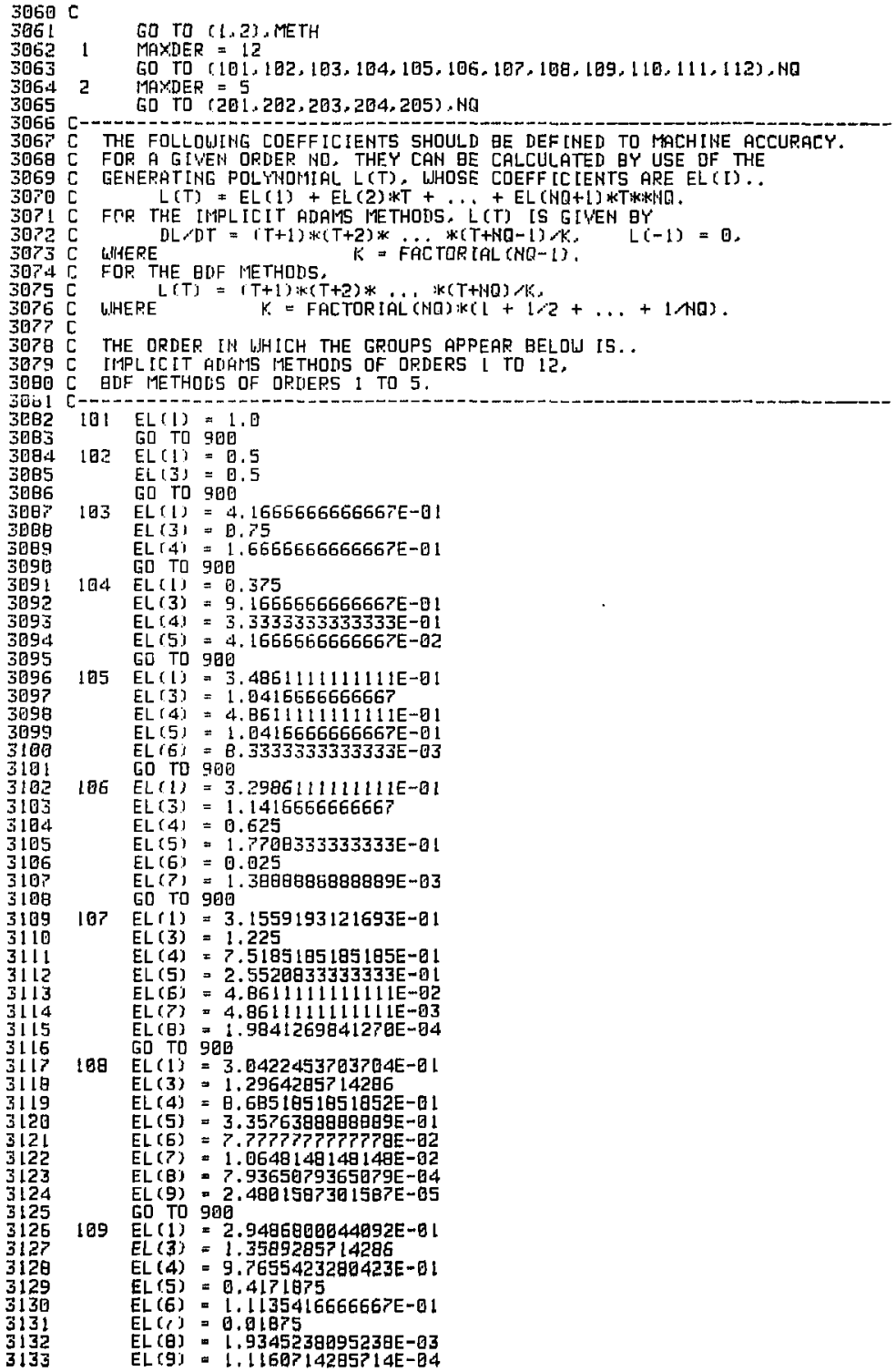




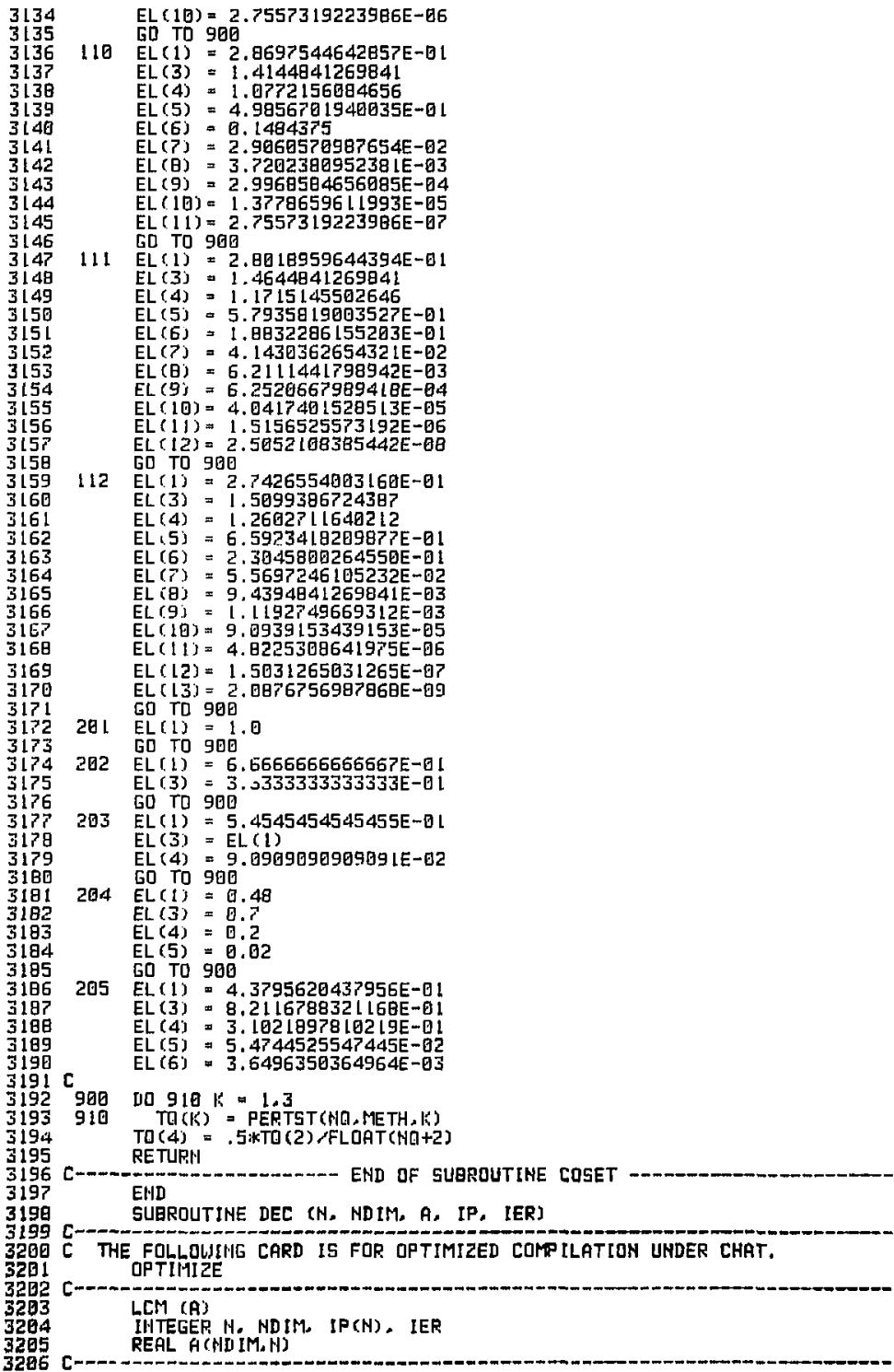


3207 C MATRIX TRIANGLULARIZATION BY GAUSS ELIMINATION WITH PARTIAL PIVTIMG.

3200 C INPUT.

$\mathrm{H}=$ DRDER DF MATRIX.

HDIM = DELLARED FIRST DIMENSIOH OF ARRAY A.

$A=$ MATRIK TO BE TRIANGULAR IZED.

OUTPUT. .

A $[I, J), I . L E, J=$ UPPER TRIANGULAR FACTOR, U

A $(i, J)$, I.GT.J = MULTIPL IERS a LOUER TR IÁNGULAR FACTOR, $[-L$.

IP(K), K.LT.N = INDEX OF K-TH PIYOT ROU.

IER = O IF MATRIX A IS NDNSINGULAR, OR $K$ IF FOUND TO SIHEULAR AT STAGE $K$.

ROW INTERCHAHGES ARE FIYISHED IN U. ONLY PARTLY IN L.

USE SOL TO OBTAIN SOLUTION OF LINEAR SYSTEM.

IF IER . ME. Q, A IS SINGULAR, SOL UILL DIVIDE BY ZERO.

CLASS DHE ROUTINE: DEC

DATE LAST CHASION: PELEASE STATUS: UIAL IMITED

EACH CLASS OHE ROUTIHE HAS BEEH THOROUGHLY TESTED BY HMG AND REETS CERTAIN LOCUMENTATIOH AMD PROGRAMMING STANDARDS.

AT LEAST ONE CONSULTAHT IS AVAILABLE TO ANSWER OUESTIONS AND RESPDND TO REPORTED ERRORS OR INADEOUACIES IH A CLASS OHE ROUTIHE.

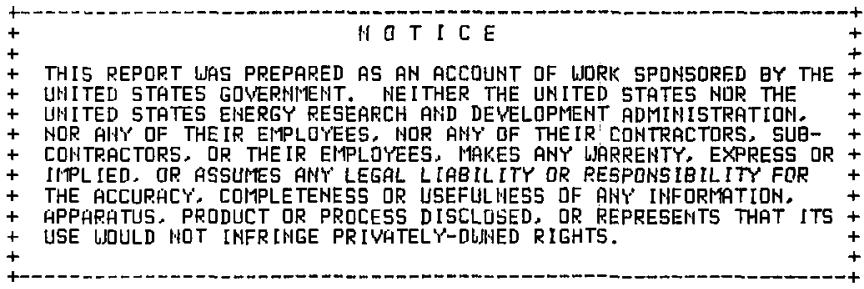

PLEASE F.EPORT AHY SUSPECTED ERRORS IH THIS ROUTINE IMHEDIATELY TO MME.

$325 \mathrm{~B} C \mathrm{CL}$

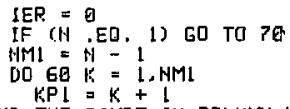

C FIND THE PIVOT IN CQLUMN $K$. SEARCH ROLS $K$ TO $N$. $M=K$

DQ $181=K P I, H$

10 IF (ABS(A $(I, K))$, GT. ABS $(A(M, K))) M=I$ $I P(K)=M$

C IHTERCHFHGE ELEMENTS IH ROLS $K$ AHD $M$. $T=A(M, K)$ IF (MI.EQ, K) GO TO 20

$A(M, K)=A(K, K)$

$R(K, K)=T$

20 IF (T,EQ, O.) GO TO 80

C STORE MULTIPLIERS IH A $(I, K), !-K+1, \ldots . .$. $T=1,2 T$

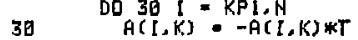


$32 B 0$ C APPLY MLLTIPL IERS TO OTHER COLUMNS OF $A$

$32 B 1$

$32 \mathrm{B2}$

$32 B 3$

3284

3285

3206

$320 ?$

32 日

3289

3290

3291

3292

3293

3294

3295

3296

3297

$329 \mathrm{~B}$

3299

3300

3301

3302

3303

3304

3305

3396

3307

3308

3309

3310

3311

$3312 \mathrm{C}$

3313

3314

3315

3315

3317

33 L

ริง 19

\$5.

3321

ง่ง 22

3323

3324

3325

5326

33구

ث328

3329

3330

3331

3332

3333

3334

3335

3356

3337

3338

3339

3340

3341

3342

3343

3344

3345

3346

3347

3340

3349

3350

3351

3552

DO $50 \mathrm{~J}=\mathrm{K} P \mathrm{~L}, \mathrm{~N}$

$T=A(M, J)$

$A(M, J)=A(K, J)$

$H(K, J)=T$

[F (T, EQ, O.) GO TO 50

DO $40 \mathrm{~L}=\mathrm{K} P \mathrm{PL}, \mathrm{M}$

$40 \quad A(I, J)=A(I, J)+A([, K) * T$

5E COHTINUE

COH

$30 \quad K=1$

IF (A (H, H) . EQ, O.) GO TO 00

RETURH

B日 IER $=K$

RETURH

[-1 END OF SUARQUTINE DEC

END

SUBROUTINE SDL (N, NDIM, $A$, B, IP )

THE FULLOUIHE CAPD IS FDR OPTIMIZED COMP ILATION UNDER CHAT. OPTIMIZE

LCH (A)

IHTEGEP H. NDIM, IP (N)

PERL H(HD [M. HJ, B G H

SOLUTIOM OF LIHEHR STSTEM A $*$ = B USING OUTPUT OF DEC.

INPUT.

$\mathrm{A}=$ DRIIER OF MATR IY.

MDIM = DECLAREII F IRST DIMEKSLIDH OF ARRAY A'.

$A=$ TR LAMELLARIZEII IATR IX OBTAIMED FRDM DEC '.

$B=$ RIEHT HAHD SIDE VECTDR.

IP = PIVOT INHFORIMATIOH WECTOR DBTAINED FROM DEC.

IID HOT USE IF DEC HAS SET IER. ME. $\theta$,

OUTPUT.

$B=$ SOLUTION WECTOR, $x$.

IF (N . EO. 1) EO TO 50

HN11 $=H-1$

C APPL $\%$ ROH PERIUTATIOLS AHD MULTIPLIERS TD $B$.

DO $20 K=\mathrm{K}, \mathrm{H} H \mathrm{H}$

$|R P|=k+1$

$19=[P$ CK. $]$

$T=B(M)$

$\theta(1)=\theta(k)$

$B(K)=T$

110 $10 \mathrm{I}=\mathrm{K}$ P $1, \mathrm{H}$

$19 \quad \theta([)=B(I)+A([, K) * T$

20 COHT [NUE

BACK SOLVE.

$D O \quad 40, K B=1, H M 11$

$|K| 1 \mid=H-K B$

$K=k 11+1$

$B(k)=B(k) / A(K, k)$

$T=-B(K)$

Do $30 I=1,1 \mathrm{H} 1$

$30 \quad \theta(D)=B([)+A(I, K): k T$

40 COHTINUE

$B(1)=B(1) / A(1,1)$

RE TURN

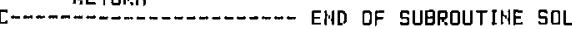

END

SUGRQUTLHE TYPES

SUARDUTLHE PIPE

THIS MODULE REPRESENTS A FISED OR VARIFBLE TIME DELAY

EQU IPMENT PARAMETERS

1 - TLAC - LENGTH OF TIME DELAY, IF TIME DELAY IS FIXED

- Negative gF VDLUME OF DELHYIHE EQUIPMENT OR PIPELINE.

2 - BYP - FRAETIOH DF STREAH HOT IIELHYED, I.E. . AYPASSED 


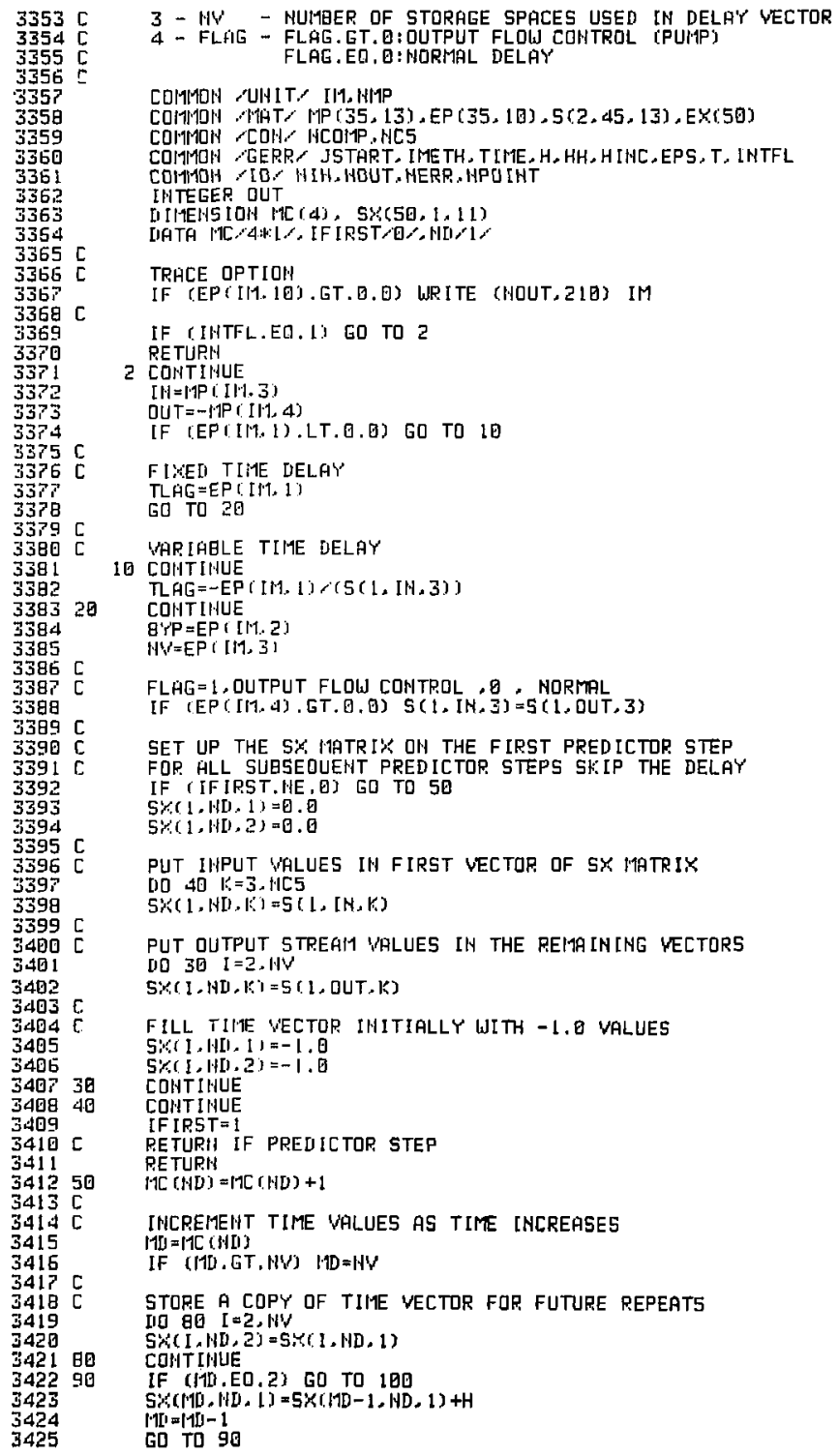




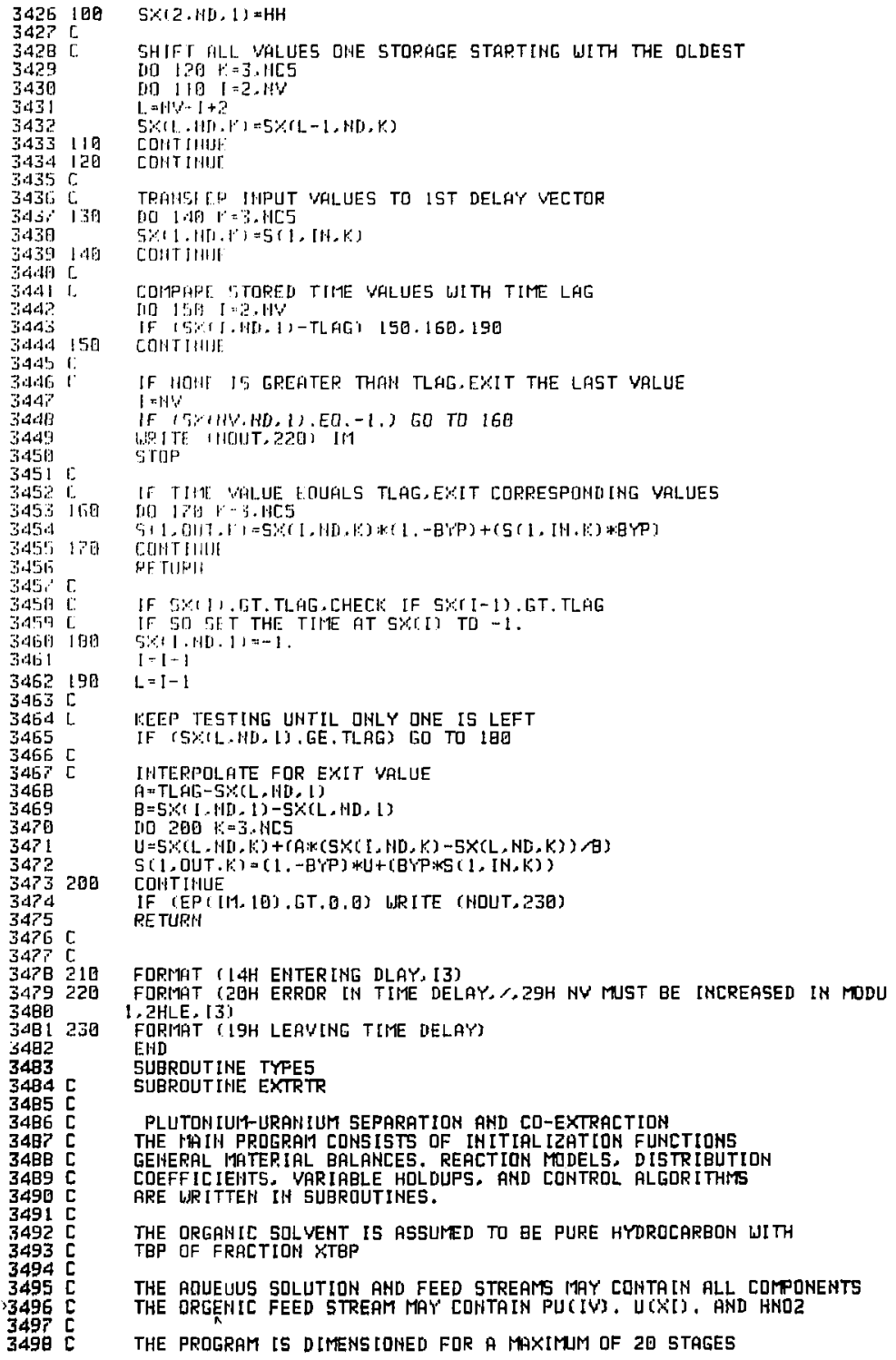




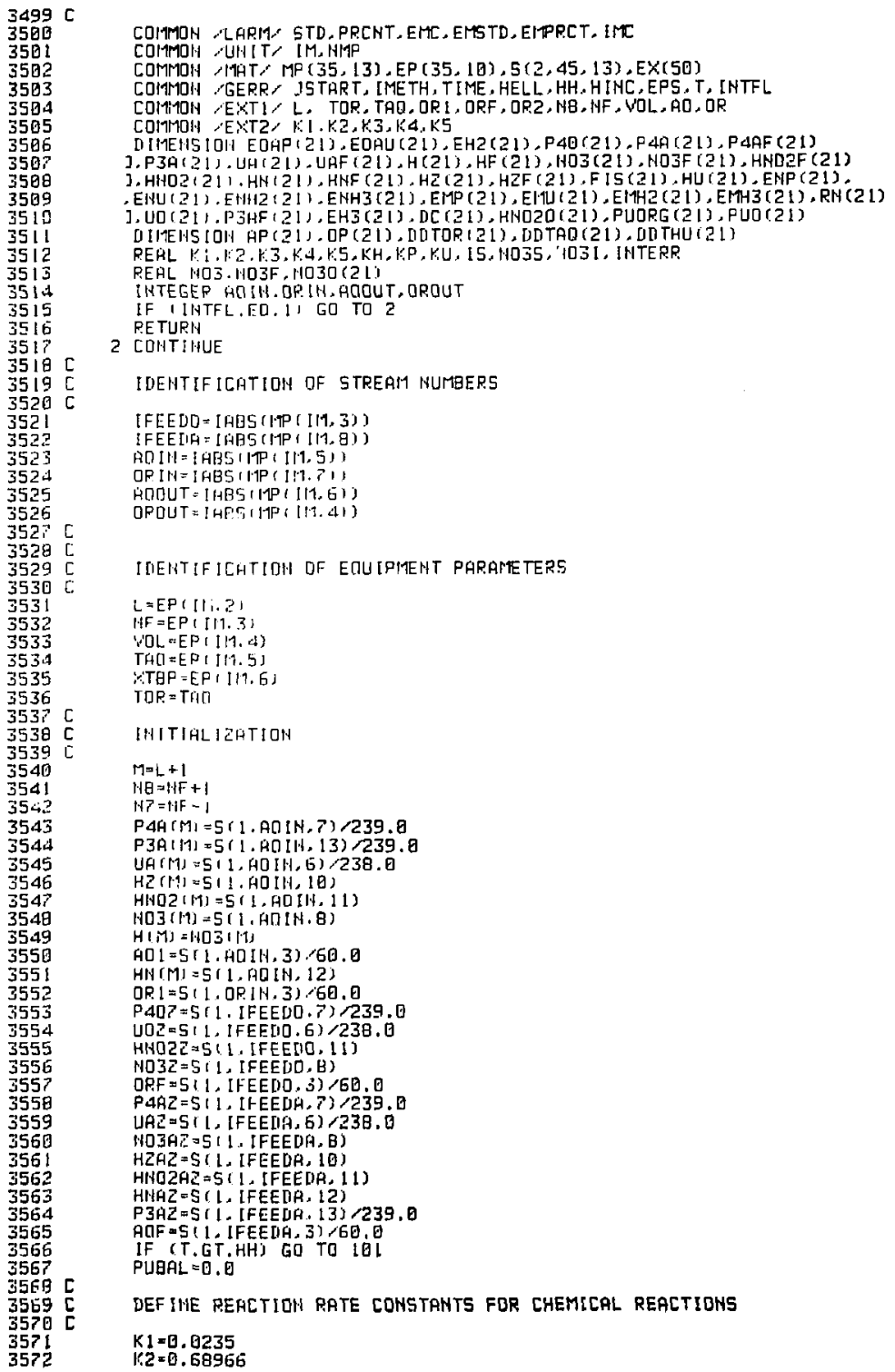




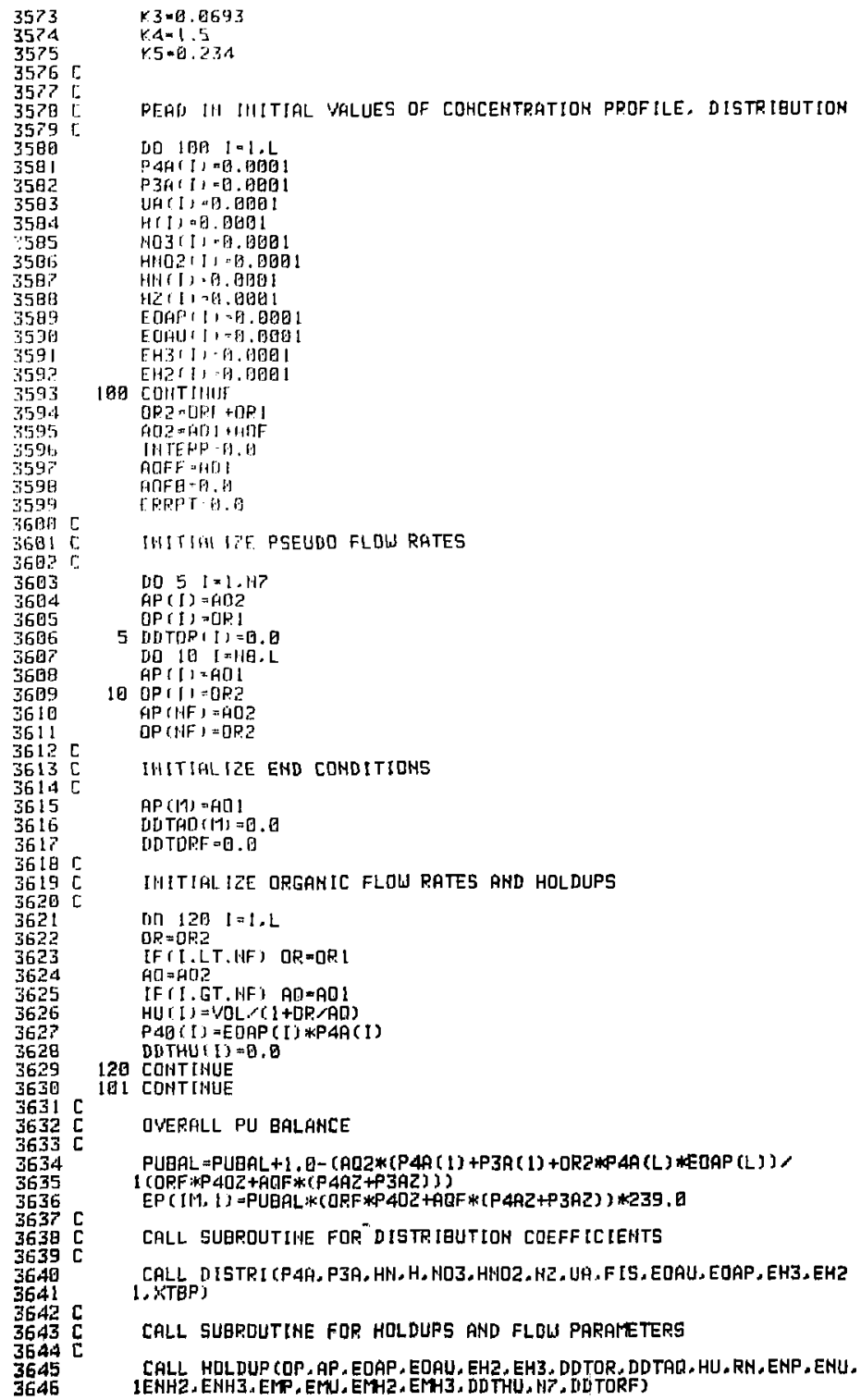




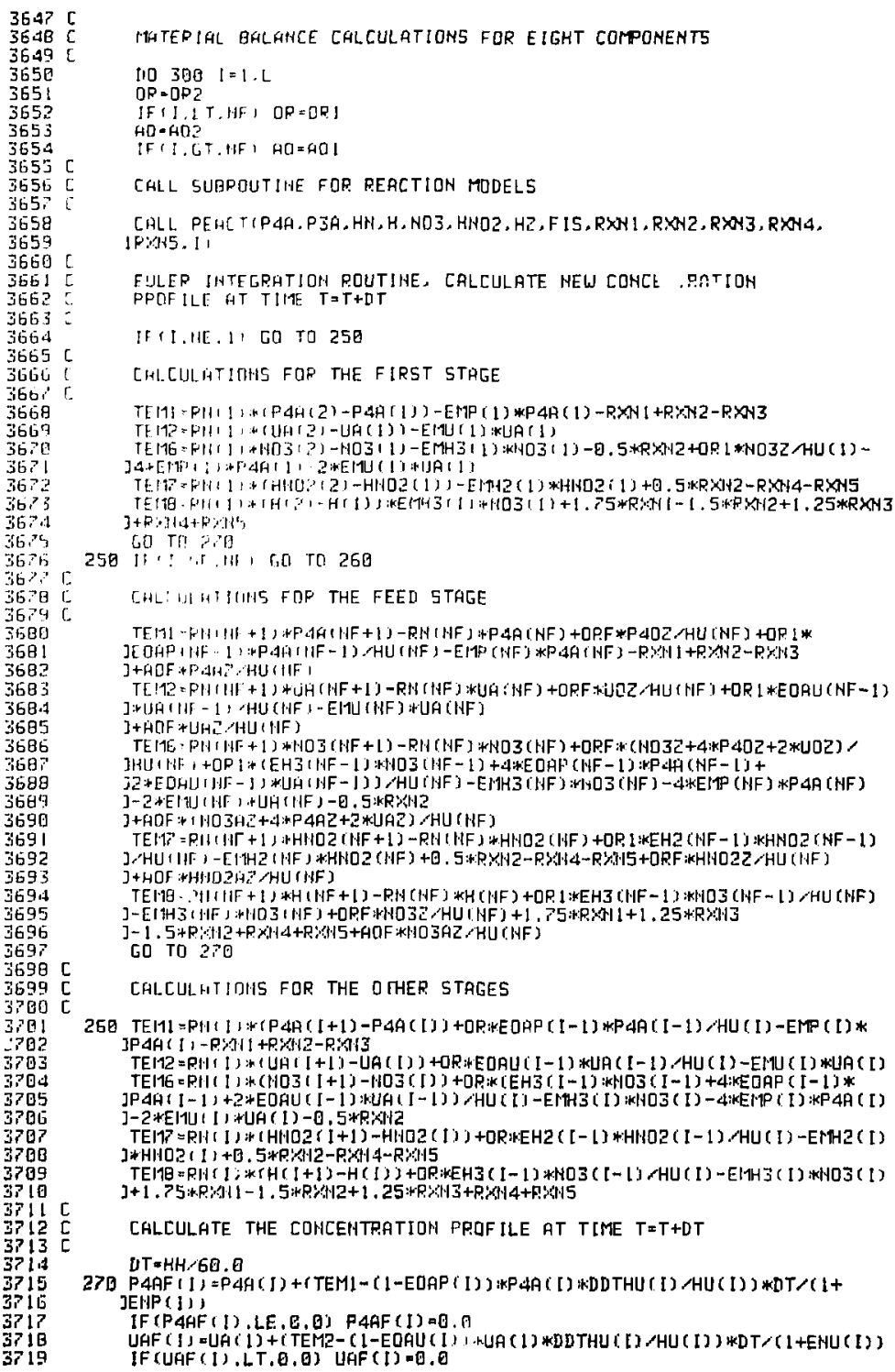




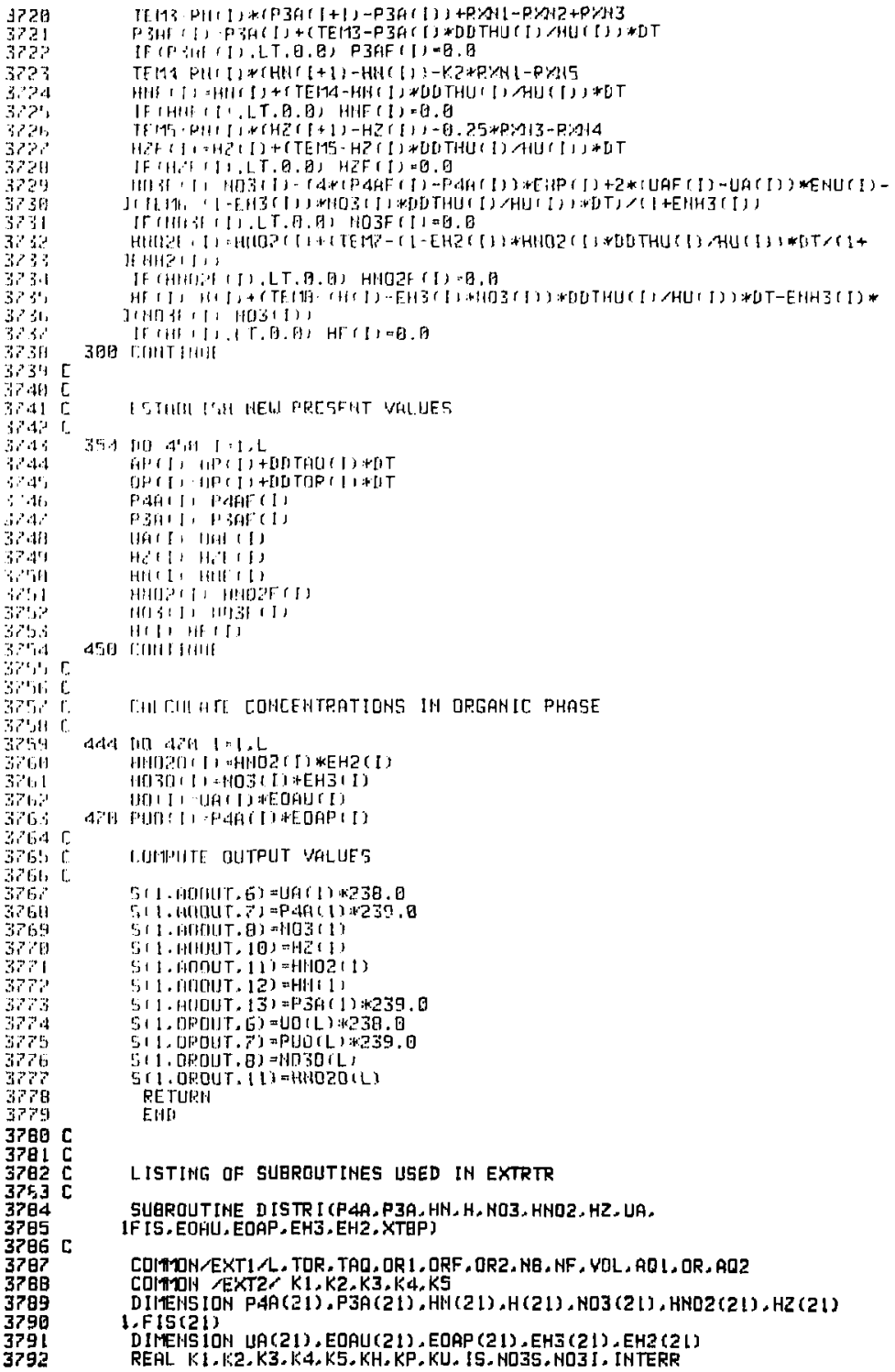


PEAL NO3, HO3F, $1030(21)$

il $150 \quad 1=1.2$

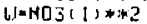

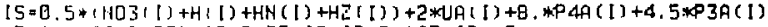

$Y P=1 ? .163-9.033 * 15+2.23 *[5 * 15 \sim 0.163 * 15 * 0 * 3$

$1 U=0.79 !+6,071+15-5.175 *[5 * 15+1,579 * 15 * 03$

$K H=0,365-0,155+15+0,024 * 15$ is

$F(S(I)=10 * *(0.91 * S O R T($ IS $)-1.52 !)$

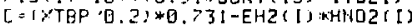

H $]=I+H+\mathrm{H}(1) * \mathrm{~N}(0311)$

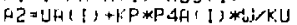

$A 3=P 4 A(11+1: U * k U A(1),(K P *(J)$

A. 4 * ( 1 ( $1403([)) * * 2$

$+5=4.61$

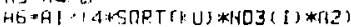

$H:=H !+1: 5(D P T(V) P)+(W: R 3)$

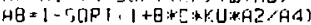

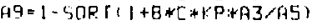

EOAU: $11=1 \mathrm{HG} * 481+* 2$

EOAP I I $=44 \vec{i} * 491+2$

B! $-\mathrm{r} H+\mathrm{H}(1+1+1 \mathrm{Mt} 3(1)$

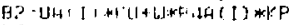

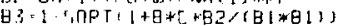

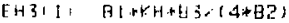

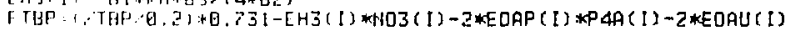

J*UHi,

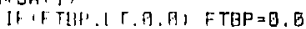

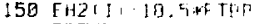

PE TtItili

Elit!

SUAPOUT INE HOL DUP (OP, AP, EDAP, EDAU, EH2, EHZ, DDTOR, DDTAD, HU, RH, ENP, IENU, FIIH? ENH 3, EMP, EITU, EIH2, EMH3. DDTHU, HP, DDTORF

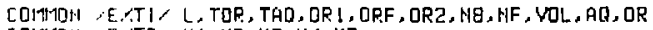

COT+1TOH, E.T2, K.1,K2,K3,K4,K5

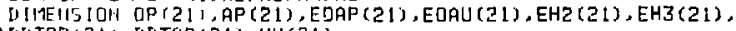

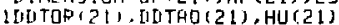

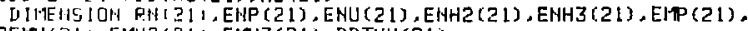

2EHU (21), EMHZ I 211, EITH3 121 ). DDTHU (21)

FEHL $r 1, K, K 3, K 4, K 5, K H, K P, K . U, 15, N O 35, H O J I$, INTERR

PEAL. $1103.1103 F, 11030$ (21)

CHLCULATE HOLUUPS HND FLDW PARARETERS

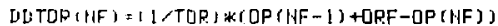

DO 160 I :, $8 . L$

160 UITOP (1 $1=(1, T O P) *(O P(1-1)-O P(1))$

DO $200 \quad I=1 . L$

$06=0,5$

IF (I.LT.IIFI QP $=0 P$ I

$\mathrm{AQ}=\mathrm{AOC}$ ?

[F I I.GT. HF ] $A O=A D]$

[HITHQI $11=11$ /TAQ 1 K(AP $(I+1)-A P(I))$

HU $[1]=10 L 11+O P(I) \cdot A P P(I))$

PHI [ $=H[, H U(I)$

ENP I I = QP (I) HEQAP (1)/AP (I)

EHU I I $]=D P($ I $) * E O A U$ ( I) $/ A P($ I)

ENHE (I) $=0 P($ I) *EHZ (1) $/ A P(1)$

ENH3 ( I) =DP ( [) *EH3 (I) /AP (I)

EIPP (I) = OR: $*$ EQAP (I)

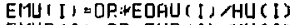

EIHA (I) $=$ OR inEHZ (I)

200 EITH3I!)=0R:

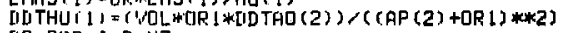

ID $302 \quad I=3.117$

2 2 DDTHU 1$)=($ VOL *OP $([-1) * D D T A G(1+1))>((A P([+1)+0 P(1-1)) * 2)$

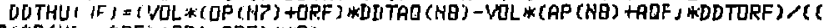

$3(A P(N)+A D F)+D R 1+D R F) * * 2)$

DD $\Rightarrow !=118 . L$

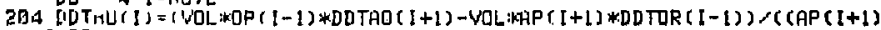
$j+D P(I-1) ;(k+2)$ R.ETURII EHD 


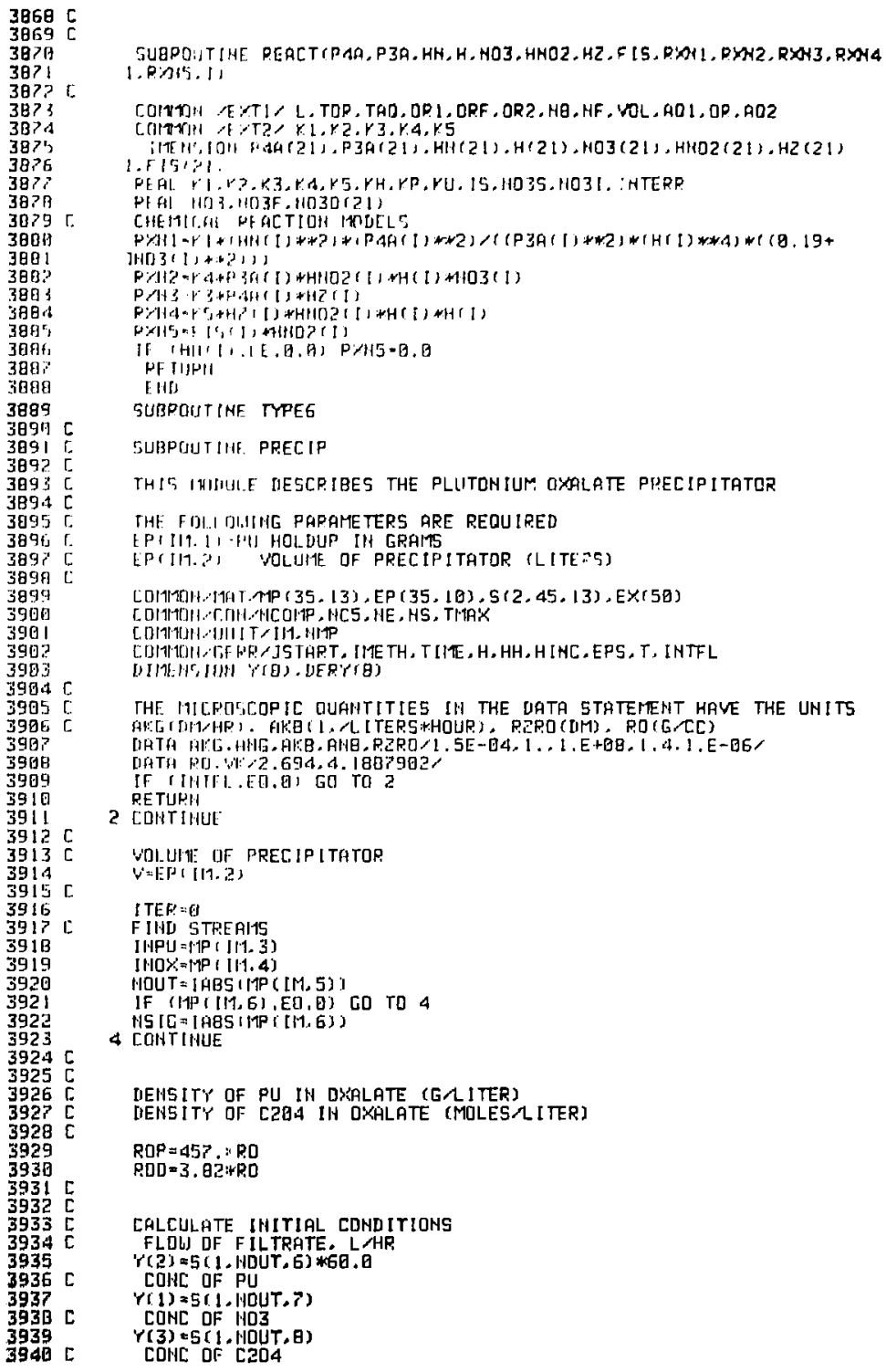

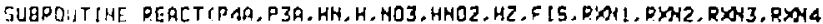

1.0.015.11

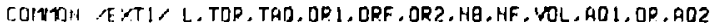

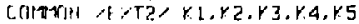

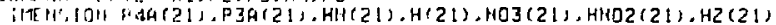
1.F $151 \% 1$.

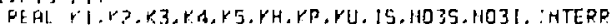

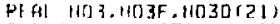

CHEHIT.G WF GCTIDH INOELS

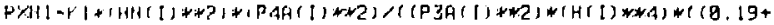
]H[3 $3(1)+4,1) 1$

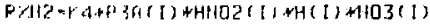

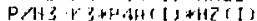

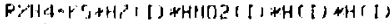

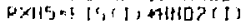

If $(H I 1,1,1 E, Q, A)$ P,

PFTIIPII

(HI)

SUBPQISTINE TYPEG

SUBPGUTIHE. PRECIP

THIS IHISUILE IIESCPIBES THE PLIITONIUM, QXALATE PIECIPITATCR

THE FDI.I IJIIIG PARAMETERS ARE REQUIRED

LPIIIT.1।-भII HOL_DUP [I GRAMT

EPrII1. I VOLUHE OF PRECLPITATOR (LITE:5)

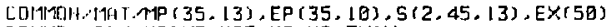

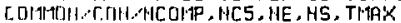

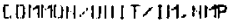

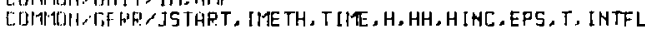

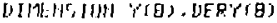

THE IILPCISCOPIC QUANTITIES IH THE UATA STRTEMENT HAVE THE UHITS

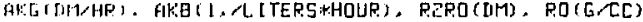

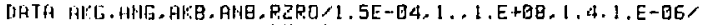

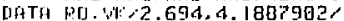

IF IIHIFI. E II, GI GO TO 2

PETURH

2 CDNTINUE

VDIJII: OJF PRECIP I TATOP

$V=E$ I ( [11.2)

ITEF: $=0$

F IHD STREAITS

IINPU $=(1 P(I 1,3)$

$I N D X=1-1 P([11,4)$

NDUT $=1 A B S ~ M A P([M, 5))$

IF $(11)(11,, 6), E Q, B)$ CO TO 4

NS [C $\approx 1$ A I ITP ( $[1,6)]$

4 CONTINHE

IJENSITY OF PU IN DS'ALATE (GAITER)

[HENSIT'Y OF CEBA IH DXHLATE MOLESAITER]

$R O P=45 ?$ : : RO

$P O D=3.92 * R D$

CALEULATE IHITIAL CDHDITIOHS

FLDU DF F ILTRATE, L HHR

$r(2)=5(1, H D U T, 6) * 60,0$

COHC OF PU

$Y(1)=5(1$, HDUT, $\vec{r})$

CONC DF HDZ

$Y(3)=G(1, H D U T, \theta)$

CDNL DF C2D4 


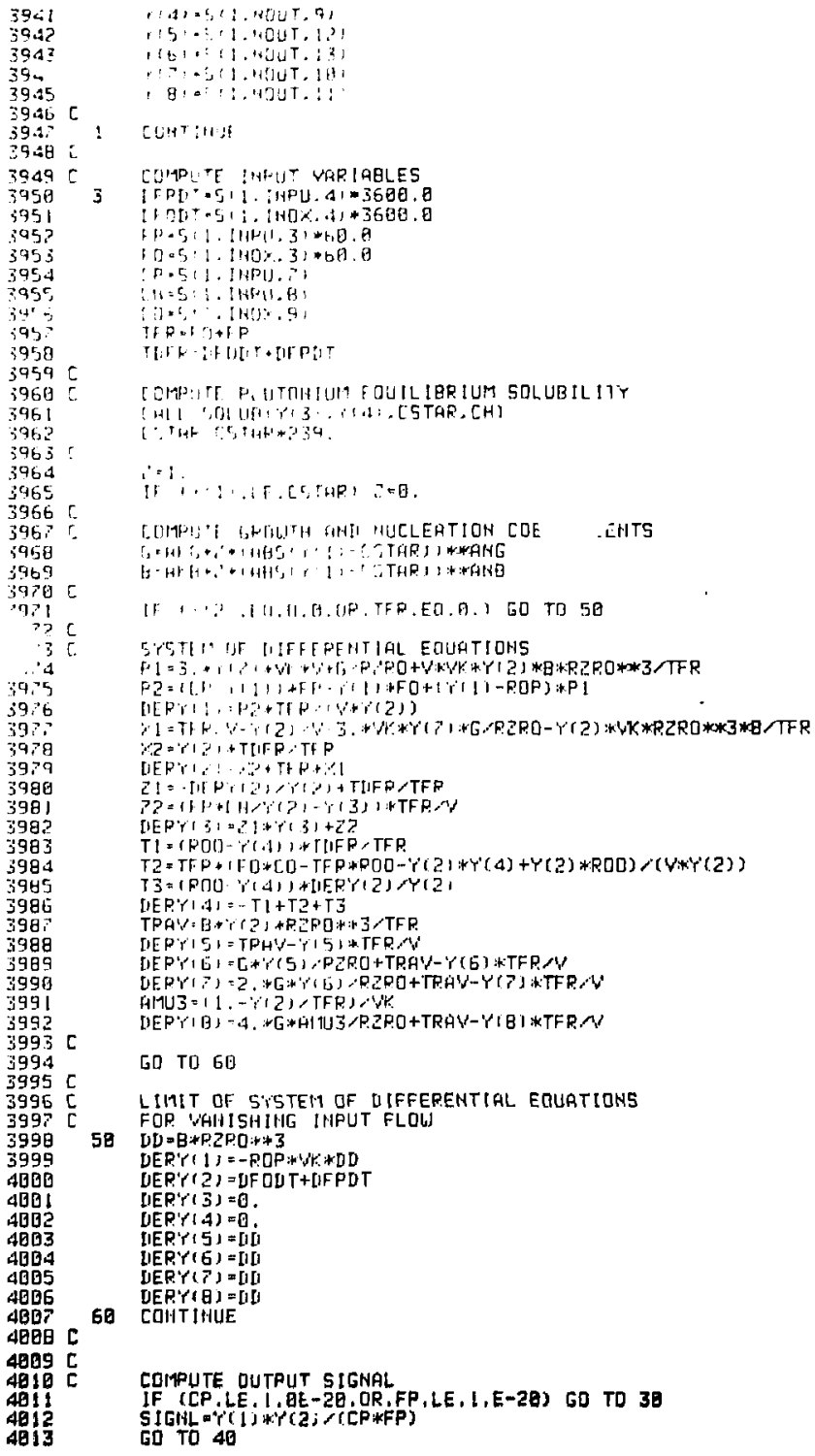




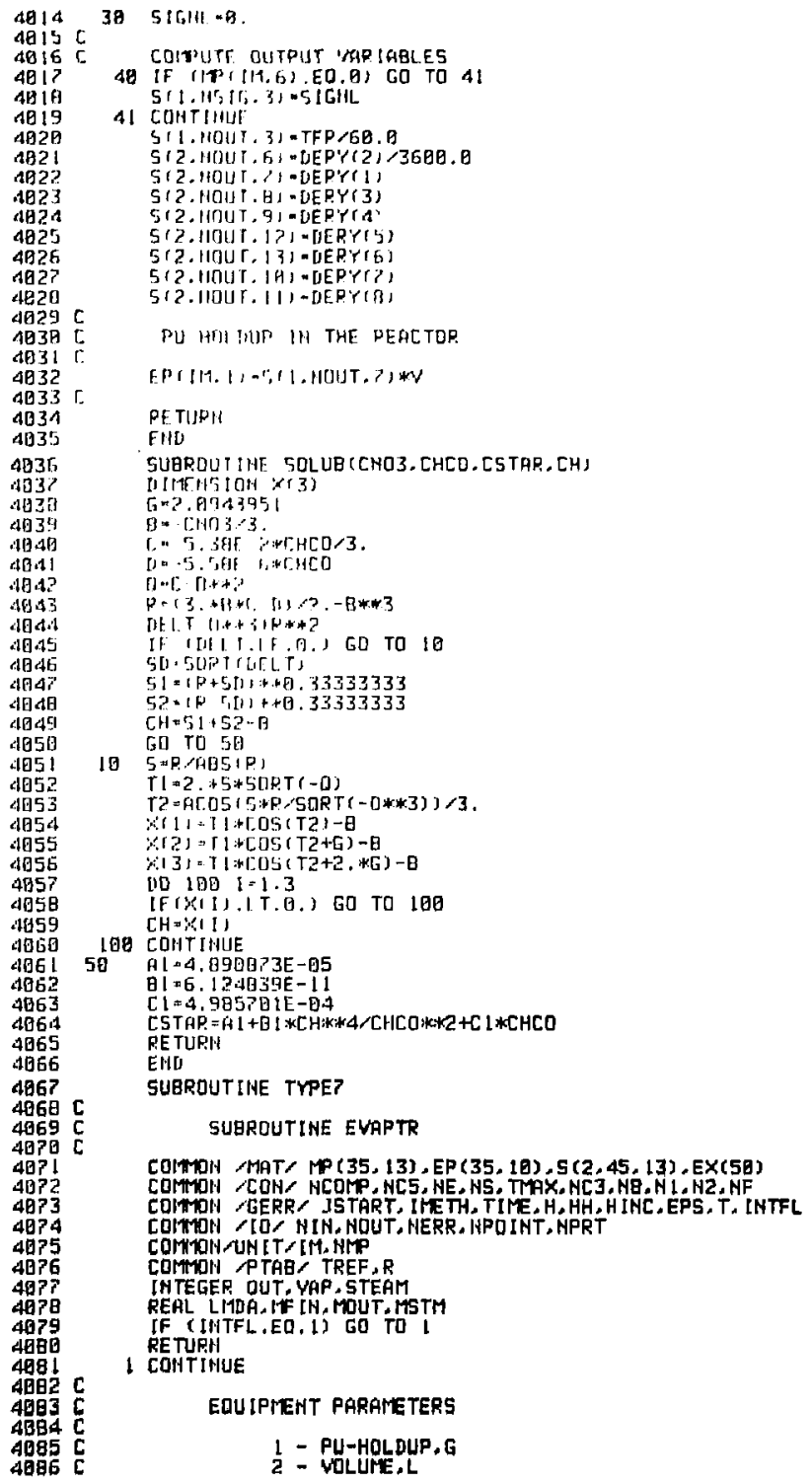




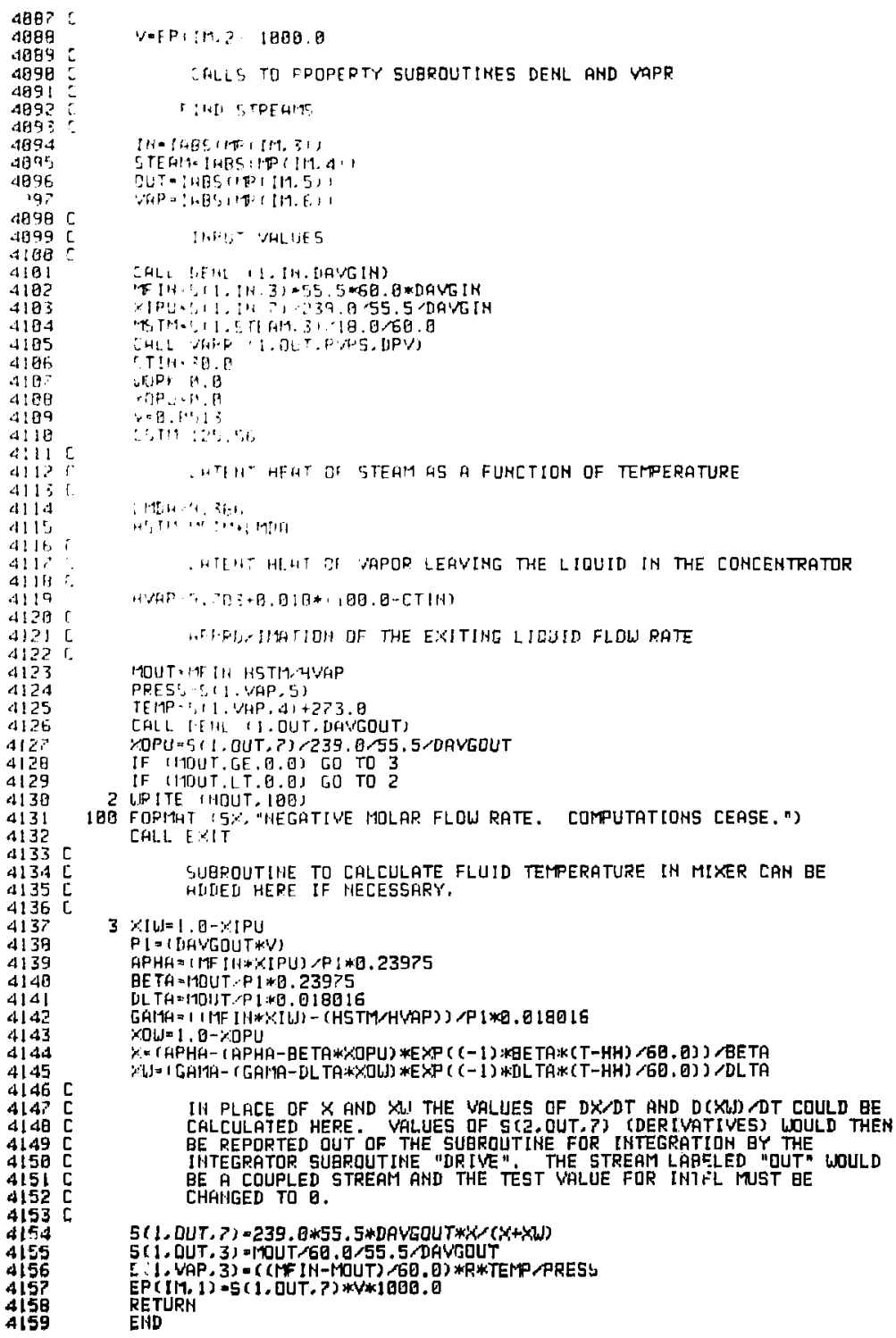




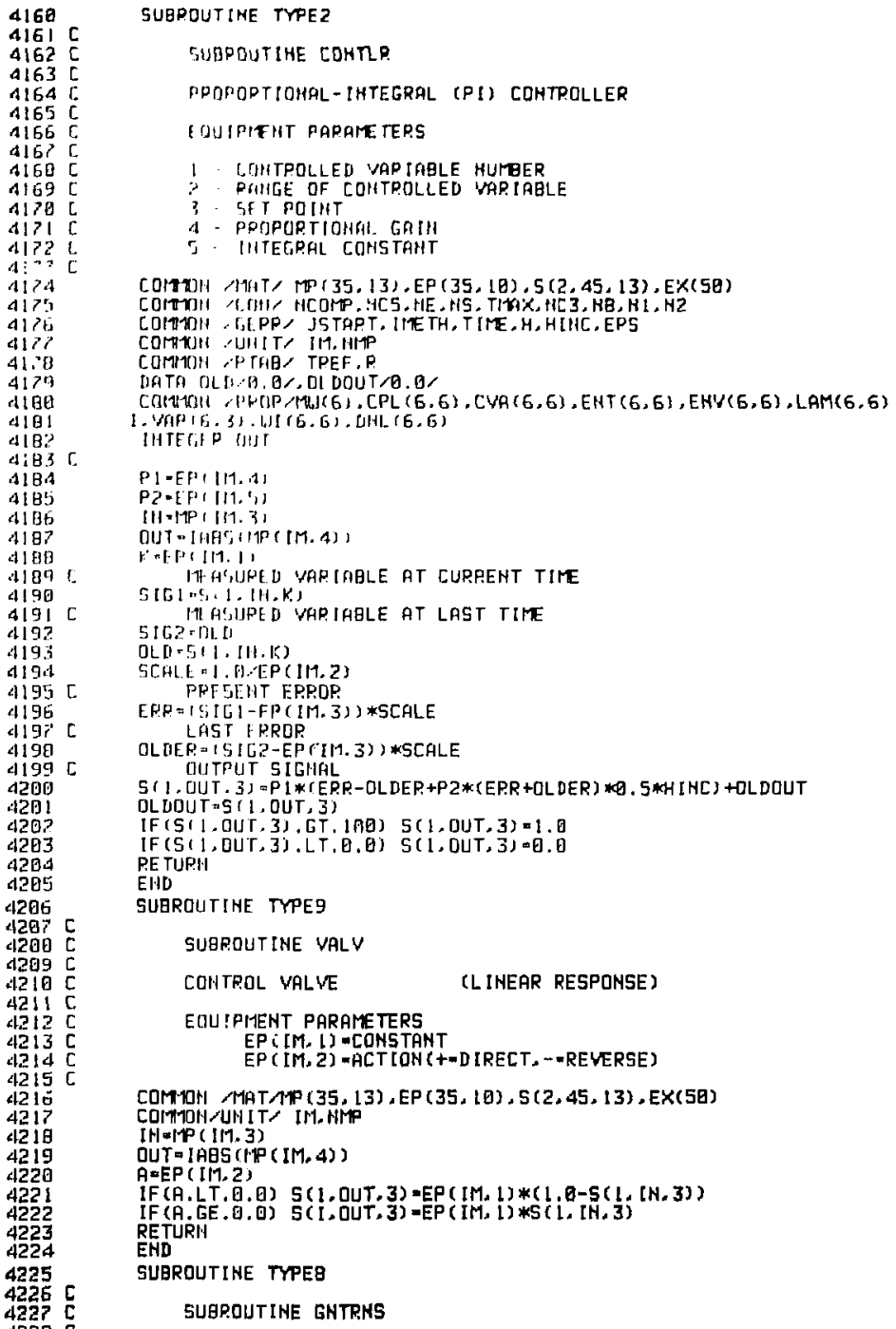

A MODULE FOR THE SIMLATION DF HEAT AND MASS TRANSFER IN A SINGLE STAGE WITH EITHER LID-LIQ OR VAPOR-LIO PHASES OR ONE WELL MIXED PHASE 


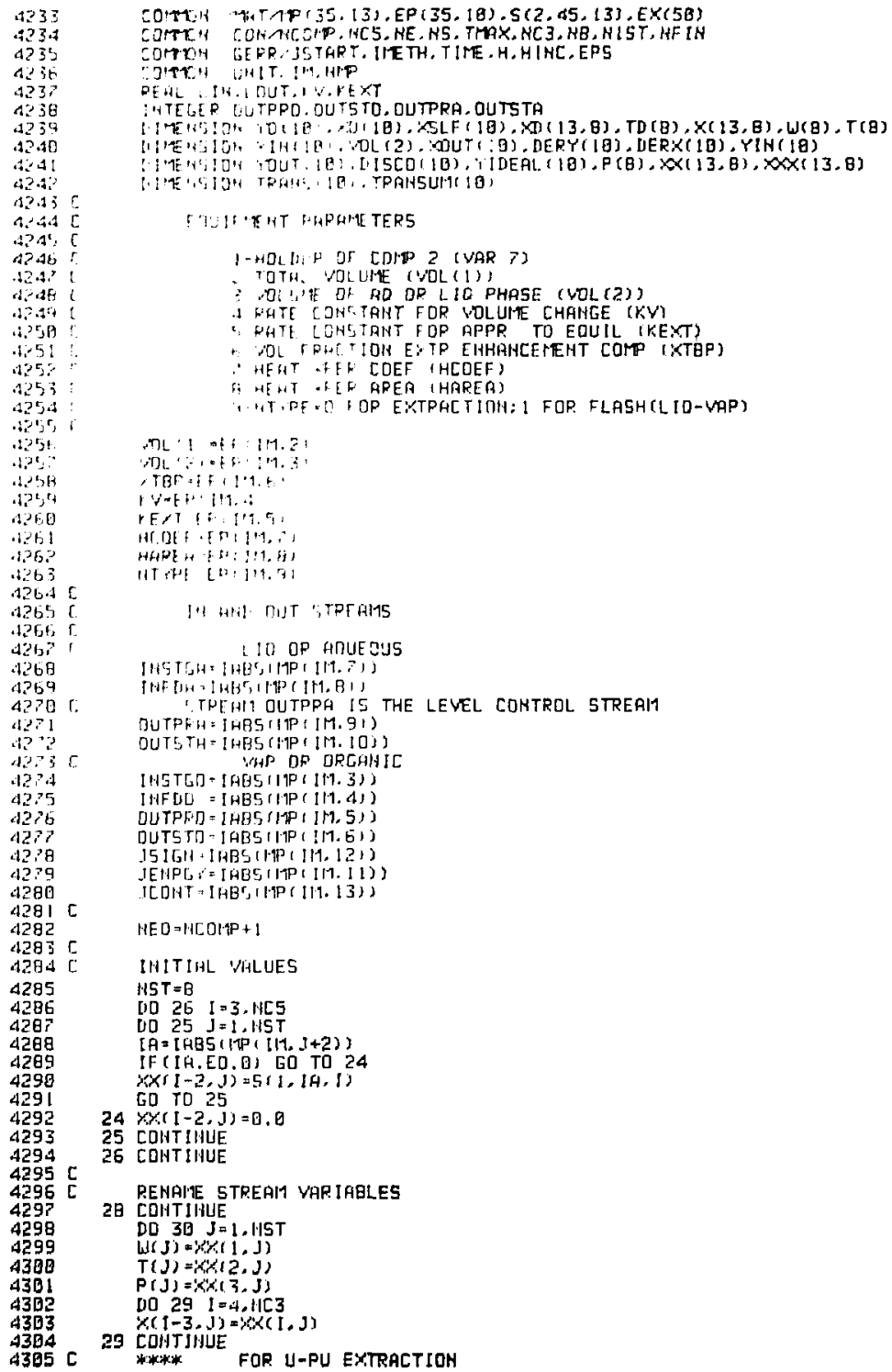


4330

4331

4332

4333

4334

4335

4336

4337

4330

4339

4340

4341

4342

4343

4344

4345

4346

4347

$434 \mathrm{~B}$

4349

4350

4351

4352

4353

4354

4355

4356

435 ?

4358

4359

4360

$4351 \mathrm{C}$

4362

4363

436 나

4365

4366

$4367^{\prime}$

4368

4369

4370

4371

4372

4373

4374

4375

4376

4377

4379

4379 $x(1, J)=x(1, j) / 238.0$

$x,(2, j)=x(2, j) / 239.0$

30 COHT [NUE

COMPUTE EMTHALPIES, HEAT CAPACITIES, DENSITIES, AND HEATS OF VAPORIZATLOM

CALL EHTL (1, INSTGA,HTL INL, DH)

CHLL EHTL (1, IHFDG. HTL IN2, DH)

CALL ENTL (1, DUTSTA.HTLDUTI,DH)

CALL EMTL (1, DUTPRA, HTLUUT2, DH)

HTL [H=HTL INI

HTLOUT $=$ HTLOUTI

IF CHTYPE, EO, OS GD TO 58

* W $(5)+H$ TL IHI2*d $(6)$

CHLL' EHTV(1, IHFDO, HTVIH2, DH)

CFLL EHTV(1, OUTSTO. HTVOUT1, DH)

CALL EHTV(1, OUTPRO, HTVOUT2,DH)

HTVIM=HTVIN I *US : +HTVIN2 KWJ(2)

HTVDUT $=$ HTVOUTI $\quad *$ *L $(3)+H T V D U T 2: k L J(4)$

5B CDNTINUE

CALL CPLI (1, INSTGA.CP5)

CALL CPLI (1, IHFUA, CPG)

CALL: CPL I (1, DUTSTA,CPQ)

CAL. L CPL I 1 , I, DUTPRA.CP7)

IF (HTYPE.EQ.Q) GO TO 59

CALL CPVA (1, INSTGO, CPI)

CALL CPYA (1, INFDO. CP2)

CALL CPVA (1, DUTPRO, CPJ)

CALL CPVA (1, OUTSTO, CPA)

59 COHT IMUE

CALL [DEHL (L, INSTGA, R.HO5)

CALL DENL (L, INFDA, RHOG)

CALL ITENL (L. OLTSTA.RHOQ)

CALL IIEHL ( L, OUTPRA,RHD?)

IF (NT'PE. EQ.O) GD TO 56

CHLL, DEHV (1, [HSTGO, RHOI)

CHLL, DENV(1, [HFDO, RHDZ)

CHLL DEHV(1, DUTPRD, RHO3)

CALL DENV(1, OUTSTO, RHOA)

5\% COHTIMUE

IF (NTYPE. HE.Q) GO TO 69

CALL DENL (L. [HSTGO, RHOL)

CALL DENL(L, [HFDO,RHO2)

CALL DEHL (1, OUTPRO, RHO3)

CALL DEHL ( L, OUTSTO, RHO4)

CALL : ENTL (1; INSTGO; HTVIHL, DH)

CALL EHTL (1. IHFDO. HTVIN2, DH)

CALL EHTL (1.OUTSTO, HTVOUTL, DH)

CALL EHTL (1.DUTPRO, HTVOUT2, DH)

CALL : CPL I ( 1, INSTGO, CP 1)

CALL, CPL [ (1, IHFDO.CP2)

CALL CPLICI, QUTSTO,CP4)

CALL CPL [ (1.DUTPRO,CP3)

69 CONTINUE

CALL LAMQ (L, OUTPRA, HTVAP)

$L[H=b](5)+b(6)$

$W(?)=5(L, J C O N T, 3)$

LOUT $=4(7)+W(8)$

CPL IN = ( L' (5) :KRHO5*CP5+W(6) *RHO6*CP G) 1 IN

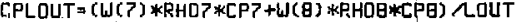

IF CVOL (1).EQ,日, Q] GO TO 41

$V I H=W(1)+W(2)$

VOUT=L.IH+VIH-LOUT

$\mathrm{U}(4)=\mathrm{VOUT}-\mathrm{U}(3)$

CPVIN $=$ ( W( 1$) *$ (

CPYOU T (U) (3) *RHOJ*KCP3+U) (d) *RHO4*CP 4) rVOUT

41 COHTIHUE

TOUT=HTLOUT (CPLOUT)

HTEXT:=HCDEF; $*$ HAFEA $*$ (TEXT-TLOUT)

DO $40 \quad I=1, H C O M P$

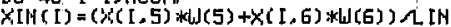

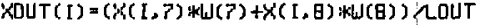




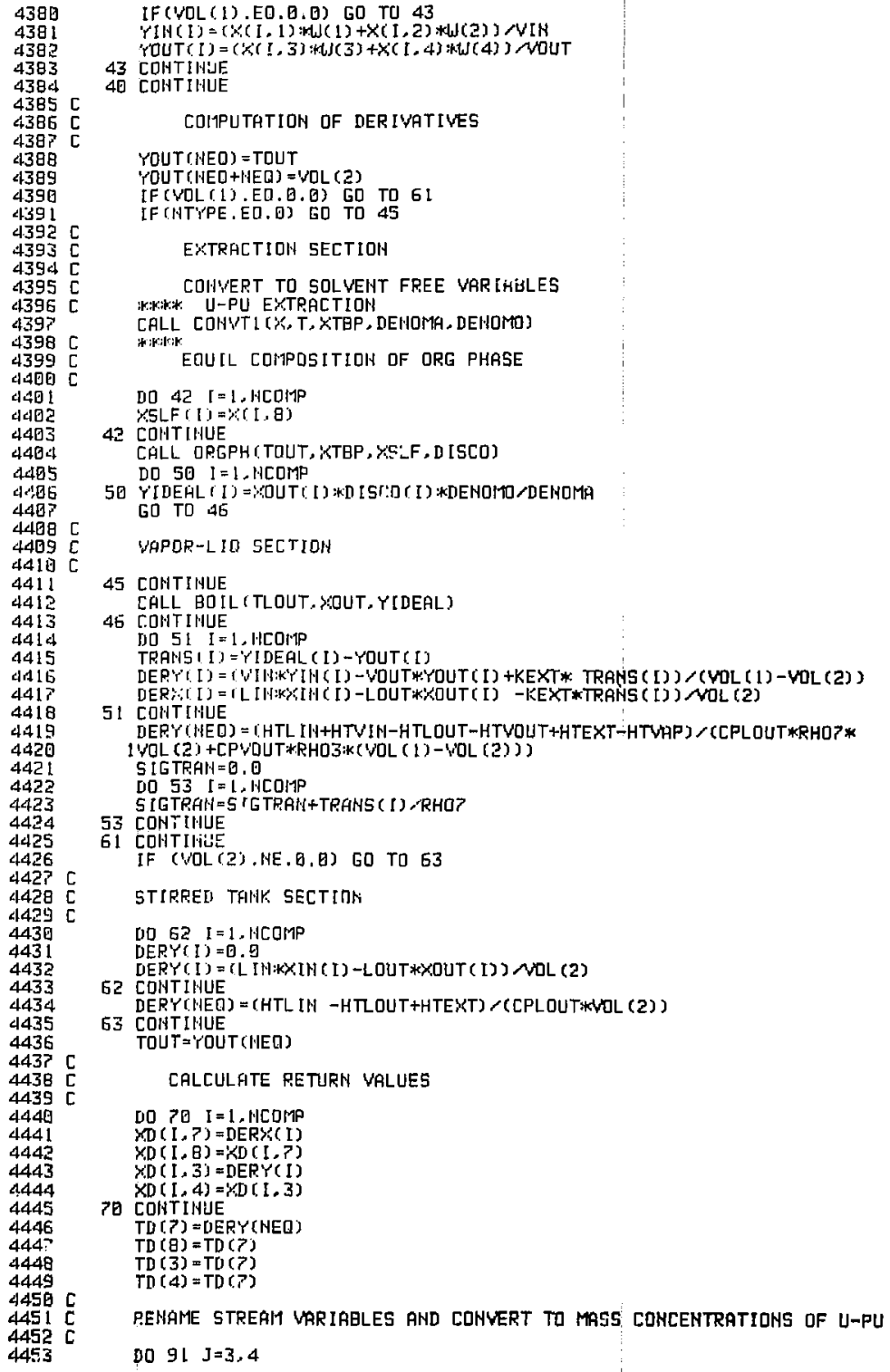

IF (VOL (1).EO.Q.0) GO TO 43

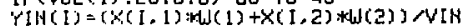

43 COHTINIE

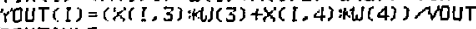

40 COHTIHUE

$$
\text { (1) }
$$

\section{COIAPUTATION OF DERIVATIVES}

YOUT (NEO) = TOUT

YOUT (HED + MEQ $=V, V L(2)$

IF (VOL (1).ED.Q.0) GD TO 61

IF (NTY'PE.Ẽ. B) GD TO 45

\section{EXTRACTIOH SECTIOH}

COWYERT TO SOLVEHT FREE VARLHULES

:FAK: U-PU EXTRACTION

CALL CONVT1 ( $\therefore, T$, XTAP, DENOMA, DEHOMTO)

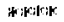
EQU CL CDHPOSITIDH OF ORG PHASE

DO $42 \quad[=1, H C O M P$

$X S L F(1)=(1, \theta)$

42 COHTIHUE

CALL ORGPH (TOUT, KTEP, YS: , D [SCO]

DO $50 \mathrm{O}=1$. HCDMP

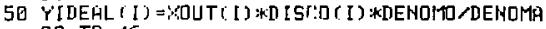
GO TO 46

VAPDR-LIO SECTIOH

45 CONTINUE

CALL BO[L (TLOUT, צOUT, YIDEAL)

46 C.OHTIHUE

ग0 $51 \quad 1=1,4 C O+1 P$

TRANS I I ) Y YIDEAL (I) - YOUT (I)

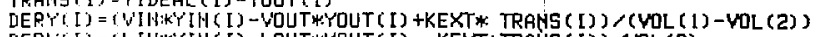

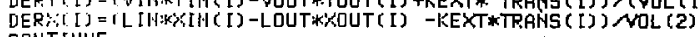

51 CONTIMUE DEP Y (HED) $=$ (HTL IN+HTVIN-HTLOUT-HTVOUT+HTEXT-HTVHP) $>$ (CPLOUT*RHO7* IVOL (2) +CPVDUT*RHOZ:K(VOL (1)-VOL (2) ) )

SIGTRAN=0,0

DO $53[=1$, HCOMP

SIGTRANISS TGTRANITRANS ( D), RHOT

53 CONTIHUE

61 Collt Iliut

IF (VOL(2), NE.0.8) GO TO 63

STIRRED TAHK SECTIDK

Do $62 I=1$ - HCOMP

DERY(I) $=0.0$

62 CDNTINUE

DERYY (HEQ $)=(H T L I H-H(T L U T+H T E S T)>($ CPLOUT $* W L(2))$

63 CONTIHUE

TOUT=YOUT (NEG)

CALCULATE RETURN VALUES

DO $70 \mathrm{I}=1$. MCDMP

$X D(I, P)=D E R X(I)$

$X D(I, B)=K D(I, P)$

$X D(1,3)=$ DERY $(1)$

$X D(1,4)=x D(1,3)$

30 CONTINUE

TD ( $P)=D E R Y(N E Q)$

$T D(\theta)=T D(7)$

$T D(3)=T D(7)$

$T D(4)=T D(7)$

PENAME STRERIT WRRIRELES AND COHVERT TO MASS COMCENTRATIOHS OF U-PU $009 \mathrm{~J}=3,4$ 


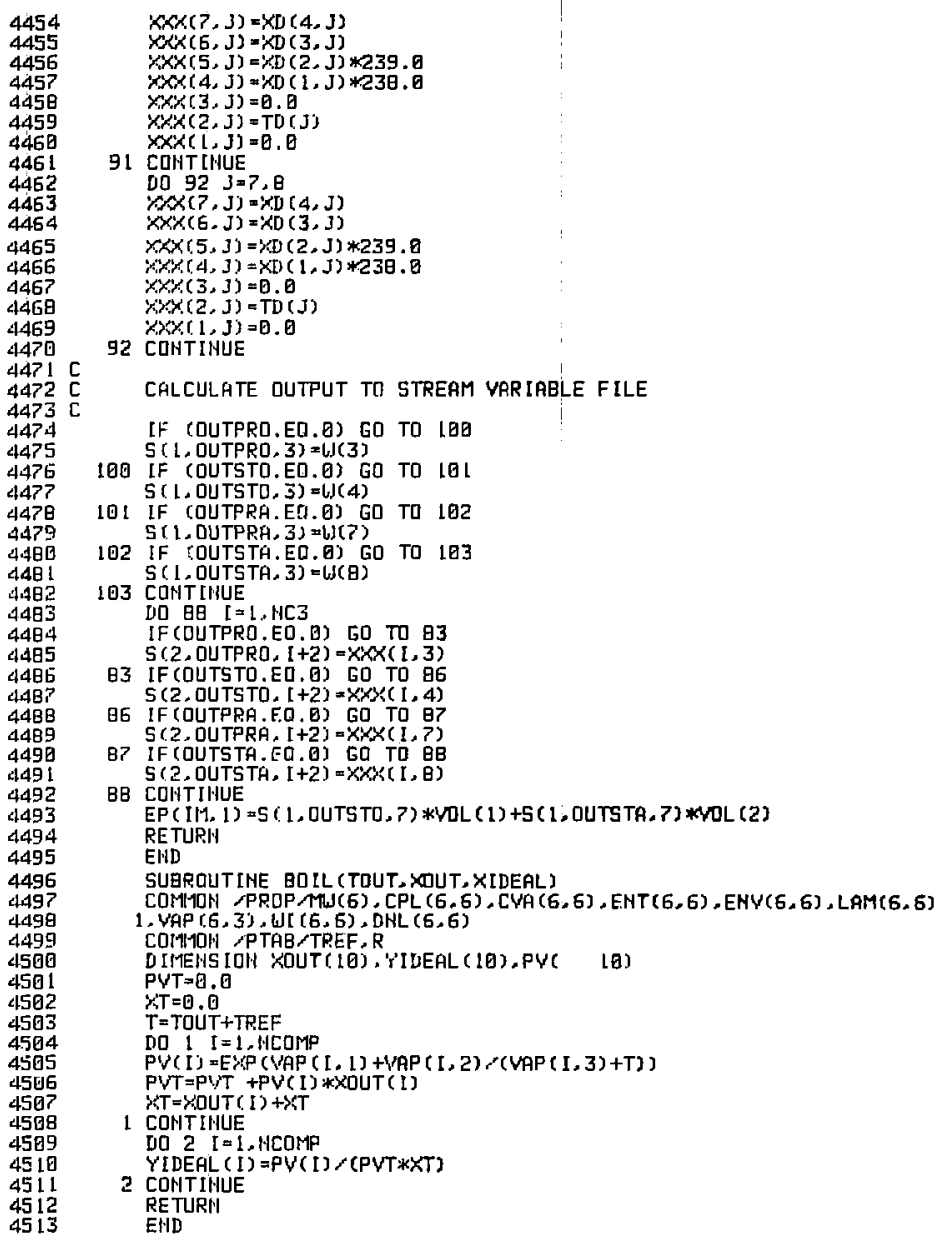




\section{REFERENCES}

1. J. R. Barney. R. S. hhluwalia, and A. I. Johnson. DYXSYS 2.0 Csers Mamal, Univ. of Western Ortario, London, Ontario (1975).

2. Cieneral Purpose Simmation System/360 OS and Do 5 Revision 2 Coers M/amal. IBM Corporation. Whitc Plains, N.Y.. No. SH20-06940 (1969).

3. A. A. B. Pritsker, The GiSP/V Simulation Langmage (John Wiley and Sons. New York, 1974).

4. P. J. Kivial. R. Villanueval, and H. Markovita, The Simscript // Programming Language (Prentice-1lall. Einglewood (Cliffs, N.J.. 1969).

5. R. L. Molitrd, PRODSC-A Simulation Program for Process W!namics and Comtrol-l sers Giatle. Dept. of Chem. and Petr. Ling.. Univ. of Houston, Texas (1970).

6. L. A. B-Lopee. DrSCO: An lerative Execuive Program for Dymanic Simulation amd comtrol of Chemical Processes. Ph.D. thesis, University of Michigan, Ann $\mathcal{A}$ rbor. Michigan (1974).

7. P. (i. Ham. W. J). Stider. and R. L. Klaus, "The Transient Analysis of Integrated Chumical Processes".

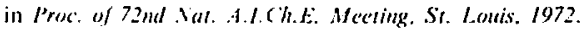

8. R. (i. 1.. Franks. Modelling and Simulation in Chemical Eingineromg (John Wiley and Sons, New York. 1972).

9. Comtinuous Sistem Hodelling Program L'sers Mamual, IBM Corporation. White Plains, N.Y., Applicition Program (iH20-0367-4 (1971).

10. Mimic-t Digital Simulation ianghage Reference Mantal. Control Datal Corp.. Minneapolis. Minnesota. Publ. No. 44610400. Rev. D. (1970).

11. S. M. Morris, 1.1.1S: Lehigh Analog Simklator (Lehigh University, Buthlehem, Pennsylvania. (1973).

12. C. W. Citatr. Numerical Initial Valuc Problems in Ordinary Differential Ealtations (Prentice-Hall. Finglewood (lifs. N.J. 1971).

13. C. W. (jear. "DI1:SL:B for Solution of Ordinary Differential Equations," (omm. of the AC:I/ /4. I85 (1971).

14. A. (C. Hindmarsh. (il:1R: Ordinary Differental Equation System Solver. Lawrenee I.ivermore l.aboratory. Rept. U(II)-30001. Rev. 3 (1974).

15. C. L. Miller. J. I:. Hammulman, G. M. Borgonoui, Dymanic Process Moded of a Plumizm Oxalate Precipituer. l.aurence L.ivernore Laboratory. Rept. UCRL-13812 (1977). 